\title{
29. HIGH-RESOLUTION GEOCHEMICAL VARIATIONS AT SITES 723, 728, AND 731: A COMPARISON OF X-RAY FLUORESCENCE AND GEOCHEMICAL LOGS ${ }^{1}$
}

\author{
Richard D. Jarrard ${ }^{2}$ and Mitchell Lyle ${ }^{2}$
}

\begin{abstract}
Geochemical logging is a routine part of the Ocean Drilling Program, yet the reliability of ODP geochemical logs has rarely been evaluated quantitatively. On ODP Leg 117, geochemical logs were obtained at Sites 723, 728, and 731 . We report here an evaluation of ODP geochemical log quality based on high-resolution sampling and X-ray fluorescence measurement of 398 core samples from the three sites.

At these sites we lacked the complete suite of high-quality logs needed for accurate log-based estimation of elemental percentages; only calcium and silicon logs had magnitudes similar to those from XRF. However, relative variations of log-based elemental abundances could be determined. Our comparisons of the XRF analyses with the character of variations in geochemical logs indicates that the reliability of ODP geochemical logs varies substantially, within short intervals and particularly between sites. In general, the geochemical logs are capable of detecting changes in formation geochemistry that are larger than the following thresholds: $2 \%$ for $\mathrm{Ca}, 2 \%-6 \%$ for $\mathrm{Si}, 0.5 \%-1 \%$ for $\mathrm{K}, 0.1 \%$ for $\mathrm{Ti}$, $0.5 \%$ for $\mathrm{Fe}$, and $0.4 \%$ for $\mathrm{Al}$. All sulfur variations observed in the XRF data, as well as many of the iron variations, were below the resolving power of the geochemical logging tools. These precisions are generally similar to those determined at the Conoco test well by Chapman et al. (1987), in spite of the very different ODP logging conditions.
\end{abstract}

\section{INTRODUCTION}

The mineralogy of deep-sea sediments contains clues to the temporal variation of climate, depositional processes, and diagenesis. The geochemistry of deep-sea sediments reflects variations in this mineralogy. Some geochemical ratios such as $\mathrm{Ti} / \mathrm{Al}$ can be directly interpreted in terms of changing source regions (e.g., Lyle et al., 1987), while calculation of normative mineralogy from geochemical data aids in determination of the geological significance of other geochemical variations (e.g., Herron, 1986). However, any geological interpretation of geochemical data is dubious, unless we have some idea of the precision and accuracy of these data.

Two primary types of geochemical data are available from the Ocean Drilling Program (ODP). First, major elements and some minor elements can be determined by X-ray fluorescence (XRF) analysis of small volumes of core (usually about $10 \mathrm{~cm}^{3}$ ). Second, abundances of up to 13 elements $(\mathrm{K}, \mathrm{Th}, \mathrm{U}, \mathrm{Ca}, \mathrm{Si}, \mathrm{Fe}$, $\mathrm{Mg}, \mathrm{S}, \mathrm{Al}, \mathrm{Ti}, \mathrm{Gd}, \mathrm{Cl}$, and $\mathrm{H}$ ) can be determined from geochemical logging.

The geochemical logs have several potential advantages over XRF analyses:

1. The logs are continuous over the entire logged interval, independent of the vagaries of core recovery and core disturbance;

2. The logs are representative, sampling a much larger volume than an XRF measurement and unaffected by preferential core recovery of certain lithologies in mixed lithology intervals;

3. Log data acquisition is very efficient (several hundred meters can be geochemically logged in the time required for only a few XRF measurements).

In comparison to XRF analyses, geochemical logs have two major disadvantages:

\footnotetext{
${ }^{1}$ Prell, W. L., Niitsuma, N., et al., 1991. Proc. ODP, Sci. Results, 117: College Station, TX (Ocean Drilling Program).

2 Lamont-Doherty Geological Observatory of Columbia University, Palisades, NY 10964, U.S.A.
}

1. Some elements can be determined by XRF analysis that cannot be determined by logs;

2. The precision and accuracy of XRF analyses are well-determined and high, while the precision and accuracy of geochemical logs are poorly known.

This paper focusses on evaluation of the reliability of ODP geochemical logs, largely by comparison to XRF measurements. We analyze and compare geochemical logs and XRF measurements from three sites drilled on ODP Leg 117: Sites 723, 728, and 731 . Sites 723 and 728 are on the Oman margin; Site 731 is on Owen Ridge. These sites were selected for study for several reasons:

1. Core recovery was unusually high at these sites, due to a combination of favorable lithologies for ODP core recovery, very calm seas during drilling, and relatively uniform and modest lithification. Sites 723 and 728 were double cored for further improvement of core recovery; however, we confine our analyses to only one hole per site, because detailed matching and correction of recovery depths between holes has not been undertaken yet for these sites.

2. Core disturbance was unusually low for the analyzed portions of these sites. All coring utilized the extended core barrel (XCB), and we have never seen XCB cores that exhibit less core disturbance than these. The core disturbance in general appears to be almost as low as with the advanced piston corer (APC); APC cores are less suitable for comparison to geochemical logs, because they are usually confined to the depth interval in which geochemical logs are obtained through pipe and therefore are less reliable than the openhole geochemical logs considered here.

3. Geochemical logs were obtained from all three sites. Though routine in ODP, geochemical logging is not ubiquitous. Unfortunately, the complete suite of geochemical logs was obtained only at Site 723 , as described in a subsequent section.

4. Substantial geochemical variability occurs within each of the three sites, at a depth scale large enough to make individual XRF measurements representative and to avoid averaging of major geochemical variations by individual log measurements. This conclusion was based on visual core descriptions; it is confirmed by the analyses reported here. 
5. One of the authors (RDJ) participated in Leg 117, thus gaining access to cores and logs and obtaining familiarity with sometimes inadequately documented aspects of coring and logging quality and operations (e.g., the core swelling problem at Site 723).

6. Because of the major effort involved in obtaining sufficient XRF analyses for the core/log comparisons which are the primary focus of this project, it is desirable for the XRF analyses to be scientifically useful in themselves. Although not addressed in this paper, the paleoceanographic value of XRF analyses at these sites is substantial.

This evaluation of geochemical logs by comparison to XRF analyses is not the first such test. The precision and accuracy of the spectral gamma tool $(\mathrm{K}, \mathrm{Th}$, and $\mathrm{U})$, gamma spectroscopy tool $(\mathrm{Ca}, \mathrm{Fe}, \mathrm{Si}, \mathrm{S}, \mathrm{Ti}, \mathrm{K}, \mathrm{Gd}, \mathrm{H}$, and $\mathrm{Cl})$ and aluminum clay tool were established at the Conoco test well by comparison to neutron activation analyses of cores (Chapman et al., 1987). A recent symposium on geochemical well logging (Schlumberger, 1988) cites results of similar studies at several other petroleumindustry wells. Anderson et al. (in press) compare XRF and geochemical log data for four scientific drill holes, of which one is an ODP hole (Hole 504B, basalts and diabases). These studies indicate a remarkably high quality of geochemical logs. For example, assuming no XRF error and no depth error at the Conoco test well, Chapman et al. (1987) estimated the following precisions (expressed as weight percent standard deviations) for individual log-based elemental concentrations: $1 \%$ for $\mathrm{Al}, 2 \%$ for $\mathrm{Ca}, 0.5 \%$ for $\mathrm{Fe}, 0.25 \%$ for $\mathrm{K}, 1.5 \%$ for $\mathrm{Si}, 1.5 \%$ for $\mathrm{S}$, and $0.1 \%$ for $\mathrm{Ti}$.

However, these published analyses are probably not appropriate for ODP geochemical logs. ODP hole conditions are quite different from those in most industry wells; in particular, ODP sedimentary sequences are usually less lithified and consequently hole diameter is much less uniform. Because the ODP gamma spectroscopy tool (GST) has an outer diameter only slightly less than the drill pipe inner diameter, ODP has been unable to utilize the boron sleeve used in industry GST logging to reduce the dominance of chlorine counts over other elemental counts. Beginning with ODP Leg 126, a thin boron sleeve is being used now; its effect has not been quantitatively analyzed. The only published quantitative comparison of XRF and geochemical-log measurements at an ODP hole (504B) indicates only fair log accuracy (Anderson et al., 1989), though most ODP Initial Reports volumes report qualitative agreement of geochemical logs with visual core descriptions.

In this paper we report the results of 398 XRF analyses from ODP Sites 723, 728, and 731. We compare the XRF-based changes in elemental abundance as a function of depth with geochemical logs, to estimate the reliability of these geochemical logs. We also examine the inter-element patterns within the XRF data and evaluate the extent to which the geochemical logs exhibit these patterns.

\section{METHODS}

\section{XRF}

A total of 403 samples, each $10 \mathrm{~cm}^{3}$, was taken from Holes 723B, 728B, and 731A. Except for the deepest 17 samples from Hole 731A and two dolomite samples from 723B, all sampling was focussed on short 5-30 m intervals with very good core recovery, with samples taken every $25 \mathrm{~cm}$ (Holes 723B and 728B) or $30 \mathrm{~cm}$ (Hole 731A). Departures from uniform sample spacing were permitted to avoid core disturbance and rare "gravy" between biscuits. However, sample locations were not chosen to preferentially sample specific lithologies; instead, this uniform very dense sampling was designed to obtain samples fully repre- sentative of the lithologic variations observed in cores, analogous to the continuous log measurements. Intervals were chosen for high resolution sampling primarily on the basis of core recovery and disturbance; a secondary criterion was avoidance of intervals that either visual core descriptions or geochemical logs suggested were homogeneous and relatively devoid of substantial geochemical variations.

At Site 723 , core recovery was severely degraded by core expansion, attributed to high dissolved carbon dioxide. Based on the very high recoveries obtained in nearby sites at which core expansion was not a problem, we assume that each core began its trip to the surface with near-perfect recovery and that the lowest sediments in the core were forced through the core catcher and lost during core expansion, while tripping to the surface and before capping on board the ship. Prior to core splitting on board the ship, actively growing voids of a few centimeters to several meters in length were produced by the degassing. Our 25$\mathrm{cm}$ post-cruise sampling of cores skipped across these voids, assuming original contiguity of the core pieces. Two sample depths are shown for each sample in Tables 1-3: an official "ODP depth" which is based on adding the current distance of the sample beneath the top of the core to the depth of the start of the cored interval, and a "true depth" which removes any core space occupied by voids. Only at Hole 723B did the ODP depth routinely differ from the "true" depth. In a subsequent section we will see one example of a core which appears to have lost recovery from the top of the core, rather than from the bottom.

Samples were prepared for analysis by freeze drying, disaggregation in a ball mill, and then pressing the powdered samples into pellets. Five samples were not subsequently analyzed; two were dolomites that resisted ball milling. Samples were not diluted prior to pressing into pellets; thus trace elements could be measured at concentrations of less than 100 parts per million (ppm). Chemical analyses were undertaken at the Oregon State University X-ray fluorescence facility, using a Phillips PW1600 $\mathrm{X}$-ray Fluorescence Spectrometer with 25 fixed element detectors and 2 scanning LiF detectors to calculate $\mathrm{X}$-ray background at each peak. Backgrounds were established from empirical relations between measured background points and background at each peak, based on a variety of blanks with different mean atomic numbers. Each batch of 19 samples included a monitor standard. After backgrounds were stripped for each sample, stripped data were normalized to the monitor standard to eliminate minor machine drift. Concentrations were calculated using the XRF11 G program (Chriss software), calibrated with over 100 geological standards and mixtures amongst standards. Precision based upon multiple measurements of an in-house standard was approximately $3 \%$ for $\mathrm{Na}$ and $1 \%$ or better for the other major elements.

Based on inter-batch comparison of the monitor standard, almost half of the trace element analyses are unreliable. Unreliable results, encompassing the deepest samples from Site 728 and all samples from Site 731, occurred only after an electrical cable replacement. Financial constraints precluded the desirable total recalibration of the system following this change. Samples with unreliable trace element abundances are indicated by an asterisk in Tables 2 and 3. Inter-batch comparison of the monitor standard demonstrated that major element analyses were not affected by this change.

Porosities of the samples were about $60 \%$ at Sites 723 and 728 and $45 \%-70 \%$ at Site 731 (Prell, Niitsuma, et al., 1989). Thus correction was warranted for pore-water sea salt, left in each sample by the drying process. The salt percentage in each sample was calculated from the chlorine concentration, using the average measured chlorinity (Prell, Niitsuma, et al., 1989) of interstitial waters at Sites 728 and 731 and the broad chlorinity-vs.-depth trend of Site 723. Elemental concentrations were 
then corrected for dilution by the salt mass. Further, $\mathrm{Na}, \mathrm{Ca}$, $\mathrm{Mg}$, and $\mathrm{K}$ sea-salt contributions were calculated and subtracted out, utilizing the shipboard measurements of concentrations of these elements in interstitial waters. Corrected XRF results for the three sites are listed in Tables 1-3. Blank spaces in the $\mathrm{Na}$ column indicate the rare cases in which the sea-salt correction produced a negative $\mathrm{Na}$ concentration. Salt percentages are also shown, to permit backtracking to raw data.

Data were not corrected for calcite dilution in each sample, but a normative calcite correction can be made:

$$
\mathrm{CaCO}_{3} \%=\mathrm{K}_{\mathrm{cc}} \times \mathrm{Ca}_{\text {tot }}-\mathrm{K}_{\mathrm{cc}} \times \mathrm{Ca}_{\mathrm{n}} /\left(1-\left(\mathrm{Ca}_{\mathrm{n}} \times \mathrm{K}_{\mathrm{cc}} / 100\right)\right),
$$

where $\mathrm{K}_{\mathrm{cc}}=2.5$ is the conversion factor from Ca\% to calcite $\%$, $\mathrm{Ca}_{\mathrm{n}}$ is the calcium percentage of the noncalcite fraction (typically $0.7 \%$ ), and $\mathrm{Ca}_{\text {tot }}$ is the total $\mathrm{Ca} \%$ measured by XRF.

Because the focus of this paper is on geochemical log comparison with cores, only the major elements from Tables 1-3 will be discussed in subsequent sections. Geochemical logs do yield concentrations for the trace elements Th, U, and Gd. However, Gd cannot be determined by XRF, and the Th and $U$ concentrations shown in Tables 1-3 are clearly near the noise level of the instrument.

\section{Logs}

The geochemical string consists of three logging tools. The natural gamma tool (NGT) measures the spectrum of natural gamma rays emitted by the formation; inversion of the spectrum yields concentrations of $\mathrm{K}(\%)$, Th (ppm), and $\mathrm{U}(\mathrm{ppm})$ (Lock and Hoyer, 1971; Serra et al., 1980). The aluminum clay tool (ACT) uses two NGT's and an intermediate californium source of neutrons; the difference between NGT spectra for irradiated and unirradiated formation is inverted for aluminum concentration (\%) of the formation (Scott and Smith, 1973). The gamma spectroscopy tool (GST) irradiates the formation with a neutron generator and measures the spectrum of immediately induced gamma rays (Hertzog, 1979). This recorded spectrum is inverted in real time for counts of $\mathrm{Cl}, \mathrm{H}, \mathrm{Ca}, \mathrm{Si}, \mathrm{Fe}$, and $\mathrm{S}$. In post-cruise reprocessing, a significant improvement in inversion quality is achieved by inverting for $\mathrm{Ti}, \mathrm{K}$, and $\mathrm{Gd}$ in addition to the six elements above. That gadolinium, present in the formation in ppm, could affect an inversion for calcium, present in percent, is initially surprising but follows from the immense neutron capture cross section of Gd, compared to that of Ca. Potassium determined from the GST is substantially lower in accuracy than that from the NGT; in practice, the NGT potassium is usually used to determine the error in GST K, and the residual GST K is repartitioned to the other GST elements. If all three geochemical tools are run and a log of photoelectric effect is also obtained, Schlumberger software also solves for $\mathrm{Mg}+\mathrm{Na}$, by attributing the difference between predicted and observed photoelectric effect to $\mathrm{Mg}+\mathrm{Na}$ (Hertzog et al., 1987). With all of the major elements thereby determined, the proprietary algorithm solves for major-element oxide dry-weight percentages.

On ODP Leg 117, the complete geochemical log suite plus photoelectric effect log was obtained only at Site 723, for the openhole interval 92-424 mbsf. At Site 728 the NGT and GST were run over the interval 0-342 mbsf; of this interval, the top $56 \mathrm{~m}$ were logged through pipe and the remainder were openhole logs. At Site 731 the NGT and GST were run over the interval 0$979 \mathrm{mbsf}$; of this interval, the top $65 \mathrm{~m}$ were logged through pipe and the remainder were openhole logs. In this paper we consider only openhole logs, which are more reliable than those obtained through pipe.

The Initial Reports for Leg 117 show geochemical data for Site 723 as weight-percent oxides. GST data for Sites 728 and
731 were shown as yields determined by postcruise 9-element inversion, because insufficient tools were run for determination of weight-percent oxides. These are two of the most common processing outputs shown in Initial Reports volumes, though sometimes only the 6-element inversion results are shown due to processing delays. However, we use a different geochemical log processing in this paper. We consider the oxide determination published for Site 723 to be unreliable for two reasons. First, the conversion from elemental yields to oxides assumed cation/ oxygen ratios appropriate for igneous rocks, not for sediments. Second, the photoelectric effect determination of $\mathrm{Mg}+\mathrm{Na}$ failed, causing the algorithm to use a default $\mathrm{Mg}$ estimation technique. However, XRD results for the site (Cremer et al., this volume) indicate only minor dolomite; the $\mathrm{Mg}$ is in clay minerals. As we shall see subsequently, $\mathrm{Mg}$ is inversely correlated with $\mathrm{Ca}$, not positively correlated as assumed by our previous oxide inversion (Prell, Niitsuma, et al., 1989).

Our geochemical log processing begins with the 9-element GST inversion. We then repartition the GST-determined $\mathrm{Cl}$ and $\mathrm{K}$. The need for $\mathrm{Cl}$ repartitioning is demonstrated by the high and predictable correlation between $\mathrm{Cl}$ and other elemental yields. For example, we have observed that the correlation coefficient between $\mathrm{Ca}$ and $\mathrm{Cl}$ yields is minus $0.3-0.5$ in a wide variety of ODP lithologies. More compelling is our observation that the slopes of regression lines between $\mathrm{Cl}$ yields and $\mathrm{Ca}, \mathrm{Fe}, \mathrm{Si}$, $\mathrm{Ti}, \mathrm{K}$, and Gd yields at Sites 723,728 , and 731 are extremely similar to the proprietary Schlumberger repartitioning coefficients for $\mathrm{Cl}$. Thus we may assume that nearly all of the smallscale Cl-yield character is a partitioning problem, and repartition it to the other elements. The broad-scale character, associated with compaction-induced porosity reduction with depth and-at Site 723-with gradual chlorinity changes, was estimated as follows: (1) Site 728: a first-degree regression of $\mathrm{Cl}$ on depth; (2) Site 723: a second-degree regression of $\mathrm{Cl}$ on depth; (3) Site 731: a linear trend for the interval 65-494 mbsf and a constant $\mathrm{Cl}$ baseline beneath 494 mbsf.

We used the Schlumberger repartitioning coefficients rather than our regression estimates of these coefficients, because a correlation of porosity with mineralogy (e.g., higher porosity clays than carbonates) could have a slight effect on our regression estimates. However, we shall see subsequently (Figs. 11-13) that XRF data exhibit little to no correlation of porosity with mineralogy.

The need for $\mathrm{K}$ repartitioning is indicated by three observations. First, a substantial portion of the $\mathrm{K}$ yields are negative (e.g., Fig. 1); this result can only be caused by inversion error. Second, $\mathrm{K}$ yields are much higher for through-pipe logs than openhole logs. Pipe is actually lower in $\mathrm{K}$ than is the formation; the observed difference results from the fact that lower $\mathrm{Cl}$ counts through-pipe cause higher apparent $\mathrm{K}$ counts. Third, the correlation between NGT K and GST K is near zero; this observation indicates that there is virtually no information in the GST $\mathrm{K}$ yield concerning actual formation variations in $\mathrm{K}$. An extreme example of this conclusion is illustrated in Figure 1, which shows $\mathrm{K}$ yields across the transition from relatively pure carbonates to terrigenous turbidites at Site 731. Based on our XRF measurements, $\mathrm{K}$ increases from an average of $0.8 \%$ to $2.6 \%$ across this transition, but no change is detectable on the GST $\log$ of $\mathrm{K}$ yield. We therefore repartitioned all of the $\mathrm{K}$ yield to the other GST elements. We did not follow the normal Schlumberger approach of repartitioning only the difference between GST K yield and predicted K yield from the NGT. To do so would be to assume that NGT K is perfectly accurate (an assumption whose validity this study tests) and would cause a ripple effect of NGT K on the GST yields of other elements, contrary to our preference for independent tests of NGT and GST accuracy. 


\begin{tabular}{|c|c|c|c|c|c|c|c|c|c|c|c|c|c|c|c|c|c|c|c|c|c|c|c|c|c|c|c|c|}
\hline $\begin{array}{l}\text { Core } \\
\text { no. }\end{array}$ & $\begin{array}{l}\text { Sec } \\
\text { no. }\end{array}$ & $\begin{array}{l}\text { Sec } \\
\text { depth } \\
(\mathrm{cm})\end{array}$ & $\begin{array}{c}\text { ODP } \\
\text { depth } \\
\text { (mbsf) }\end{array}$ & $\begin{array}{c}\text { True } \\
\text { depth } \\
\text { (mbsf) }\end{array}$ & $\begin{array}{c}\mathrm{Na} \\
(\%)\end{array}$ & $\begin{array}{c}\mathrm{Mg} \\
(\%)\end{array}$ & $\begin{array}{c}\text { Al } \\
(\%)\end{array}$ & $\begin{array}{c}\mathrm{Si} \\
(\%)\end{array}$ & $\begin{array}{c}\stackrel{P}{P} \\
(\%)\end{array}$ & $\begin{array}{c}\mathrm{S} \\
(\%)\end{array}$ & $\begin{array}{c}\mathbf{K} \\
(\%)\end{array}$ & $\begin{array}{r}\mathrm{Ca} \\
(\%)\end{array}$ & $\begin{array}{c}\mathrm{Ti} \\
(\%)\end{array}$ & $\begin{array}{c}\mathrm{Cr} \\
(\%)\end{array}$ & $\begin{array}{c}\mathrm{Mn} \\
(\%)\end{array}$ & $\begin{array}{c}\mathrm{Fe} \\
(\%)\end{array}$ & $\begin{array}{c}\text { Co } \\
(\mathrm{ppm})\end{array}$ & $\begin{array}{c}\mathrm{Ni} \\
(\mathrm{ppm})\end{array}$ & $\begin{array}{c}\mathrm{Cu} \\
(\mathrm{ppm})\end{array}$ & $\begin{array}{c}\mathrm{Zn} \\
(\mathrm{ppm})\end{array}$ & $\begin{array}{c}\mathrm{Rb} \\
(\mathrm{ppm})\end{array}$ & $\begin{array}{c}\mathrm{Sr} \\
(\mathrm{ppm})\end{array}$ & $\begin{array}{c}\mathrm{Ba} \\
(\mathrm{ppm})\end{array}$ & $\begin{array}{c}\mathrm{La} \\
(\mathrm{ppm})\end{array}$ & $\begin{array}{c}\mathrm{Pb} \\
(\mathrm{ppm})\end{array}$ & $\begin{array}{c}\mathrm{Th} \\
(\mathrm{ppm})\end{array}$ & $\underset{(\mathrm{ppm})}{\mathrm{U}}$ & $\begin{array}{l}\text { Salt } \\
(\boldsymbol{\%})\end{array}$ \\
\hline 11 & ${ }_{2}^{2}$ & 38 & $\begin{array}{l}93.08 \\
93.33\end{array}$ & $\begin{array}{l}92.74 \\
92.99\end{array}$ & $\begin{array}{l}0.41 \\
0.5\end{array}$ & $\begin{array}{l}3.25 \\
3.23\end{array}$ & $\begin{array}{l}2.96 \\
3.05\end{array}$ & $\begin{array}{l}12.6 \\
133\end{array}$ & $\begin{array}{l}0.078 \\
0.078\end{array}$ & 0.644 & 1.011 & 18.9 & $\begin{array}{l}0.281 \\
0.29\end{array}$ & 0.0223 & 0.0638 & 2.05 & 14 & 36 & 222 & 55 & 42 & 679 & 321 & 18 & 13 & 1 & 3 & 1.88 \\
\hline 11 & 2 & 63 & $\begin{array}{l}93.33 \\
93.74\end{array}$ & $\begin{array}{l}92.99 \\
93.24\end{array}$ & $\begin{array}{l}0.5 \\
0.44\end{array}$ & $\begin{array}{l}3.23 \\
3.25\end{array}$ & $\begin{array}{l}3.05 \\
3.07\end{array}$ & $\begin{array}{l}13.2 \\
13.2\end{array}$ & $\begin{array}{l}0.071 \\
0.079\end{array}$ & $\begin{array}{l}0.571 \\
0.672\end{array}$ & $\begin{array}{l}0.99 \\
1.014\end{array}$ & $\begin{array}{l}19 \\
18.4\end{array}$ & $\begin{array}{l}0.29 \\
0.294\end{array}$ & $\begin{array}{l}0.0251 \\
0.0267\end{array}$ & $\begin{array}{l}0.0636 \\
0.0636\end{array}$ & $\begin{array}{l}2.04 \\
2.16\end{array}$ & 14 & 25 & 222 & 33 & 39 & $\begin{array}{l}667 \\
658\end{array}$ & 307 & 18 & 13 & 0 & 3 & 1.69 \\
\hline $\begin{array}{l}11 \\
11\end{array}$ & 2 & $\begin{array}{l}1304 \\
133\end{array}$ & $\begin{array}{l}93.74 \\
94.03\end{array}$ & $\begin{array}{l}93.24 \\
93.49\end{array}$ & $\begin{array}{l}0.44 \\
0.45\end{array}$ & 3.15 & 3.03 & 13 & 0.078 & $\begin{array}{l}0.672 \\
0.61\end{array}$ & $\begin{array}{l}1.0 .14 \\
0.996\end{array}$ & $\begin{array}{l}18.4 \\
19\end{array}$ & $\begin{array}{l}0.294 \\
0.291\end{array}$ & $\begin{array}{l}0.0267 \\
0.0242\end{array}$ & $\begin{array}{l}0.06399 \\
0.0638\end{array}$ & $\begin{array}{l}2.16 \\
2.05\end{array}$ & $\begin{array}{l}16 \\
13 \\
13\end{array}$ & $\begin{array}{l}52 \\
29\end{array}$ & 222 & ${ }_{32}^{43}$ & 46 & 658 & 139 & $\begin{array}{l}26 \\
29\end{array}$ & 13 & 0 & 3 & 2.07 \\
\hline ii & 3 & 12 & 94.32 & 93.73 & 0.4 & 3.11 & 2.94 & 12.6 & 0.085 & 0.561 & 0.968 & 19.7 & 0.278 & 0.0218 & $\begin{array}{l}.003837 \\
0.0637\end{array}$ & $\begin{array}{l}2.03 \\
2.06\end{array}$ & $\begin{array}{l}13 \\
15\end{array}$ & $\begin{array}{l}29 \\
35\end{array}$ & $\begin{array}{l}222 \\
223\end{array}$ & $\begin{array}{l}32 \\
48 \\
48\end{array}$ & 要4 & $\begin{array}{l}6944 \\
742\end{array}$ & $\begin{array}{l}312 \\
338\end{array}$ & $\begin{array}{l}29 \\
19\end{array}$ & $\begin{array}{l}13 \\
13\end{array}$ & 2 & 3 & \\
\hline 11 & 3 & 38 & 94.58 & 93.99 & 0.39 & 2.89 & 2.92 & 12.6 & 0.085 & 0.625 & 0.972 & 19.2 & 0.28 & 0.0255 & 0.0639 & 2.1 & 16 & 52 & 224 & $\begin{array}{l}40 \\
67\end{array}$ & $\begin{array}{l}43 \\
46\end{array}$ & $\begin{array}{l}742 \\
724\end{array}$ & $\begin{array}{l}338 \\
314\end{array}$ & 19 & $\begin{array}{l}13 \\
13\end{array}$ & 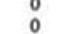 & ${ }_{3}^{3}$ & $\begin{array}{l}1.86 \\
2.1\end{array}$ \\
\hline 11 & 3 & 61 & 94.81 & 94.22 & 0.39 & 2.77 & 2.82 & 11.9 & 0.092 & 0.633 & 0.934 & 19.8 & 0.269 & 0.0238 & 0.0638 & 2.09 & 17 & 46 & 225 & 48 & 43 & 778 & 328 & 36 & 13 & 0 & 3 & \\
\hline 11 & 3 & 132 & 95.52 & 94.68 & 0.26 & 2.68 & 2.24 & 9.2 & 0.139 & 0.736 & 0.728 & 23.6 & 0.208 & 0.0177 & 0.0642 & 1.46 & 14 & 48 & 231 & 80 & 45 & 980 & 29 & 38 & 13 & 3 & 3 & $\begin{array}{l}1.88 \\
2.58\end{array}$ \\
\hline 11 & 4 & 22 & 95.92 & 95.08 & 0.26 & 2.65 & 2.27 & 9.4 & 0.143 & 0.731 & 0.715 & 23.3 & 0.209 & 0.0192 & 0.0642 & 1.5 & 13 & 56 & 231 & 89 & 42 & 971 & 92 & 27 & 13 & 0 & 3 & 2.52 \\
\hline 11 & 4 & 45 & 96.15 & $\begin{array}{l}95.31 \\
0546\end{array}$ & 0.32 & 2.8 & 2.33 & 9.7 & 0.135 & 0.691 & 0.714 & $\begin{array}{l}23.6 \\
20.6\end{array}$ & 0.215 & 0.0196 & 0.0641 & 1.46 & 11 & 52 & 231 & 90 & 39 & 84 & 54 & 27 & 13 & 0 & 3 & 2.44 \\
\hline 11 & 4 & $\begin{array}{r}70 \\
109\end{array}$ & $\begin{array}{l}96.4 \\
9679\end{array}$ & $\begin{array}{l}95.56 \\
95.81\end{array}$ & $\begin{array}{l}0.38 \\
0.48\end{array}$ & $\begin{array}{l}2.96 \\
3.07\end{array}$ & $\begin{array}{l}2.5 \\
2.6\end{array}$ & 10.5 & 0.133 & 0.6 & 0.772 & $\begin{array}{r}22.6 \\
21.6\end{array}$ & 0.238 & 0.0 & 0.0639 & 1.59 & $?$ & 44 & 228 & 71 & 42 & 86 & 145 & 27 & 13 & 3 & 3 & 2.07 \\
\hline II & 4 & 109 & $\begin{array}{l}96.79 \\
97.99\end{array}$ & $\begin{array}{r}95.81 \\
9605\end{array}$ & 0.4 & 3.07 & 2.6 & 11.1 & 0.139 & 0.603 & $\begin{array}{l}0.817 \\
0.83\end{array}$ & $\begin{array}{r}21.6 \\
21.6\end{array}$ & 0.25 & 0.0262 & 0.0639 & 1.7 & 5 & 43 & 227 & 55 & 40 & 79 & 168 & 20 & 13 & 0 & 3 & 2.04 \\
\hline $\begin{array}{l}11 \\
11\end{array}$ & $\begin{array}{l}4 \\
5\end{array}$ & $\begin{array}{r}133 \\
10\end{array}$ & $\begin{array}{l}97.03 \\
97.3\end{array}$ & $\begin{array}{l}96.05 \\
96.02\end{array}$ & $\begin{array}{l}0.39 \\
0.41\end{array}$ & $\begin{array}{l}3.19 \\
3.97\end{array}$ & $\begin{array}{l}2.62 \\
2.68\end{array}$ & $\begin{array}{l}11.1 \\
11.2\end{array}$ & $\begin{array}{l}0.143 \\
0.122\end{array}$ & $\begin{array}{l}0.575 \\
0.534\end{array}$ & $\begin{array}{l}0.83 \\
0.835\end{array}$ & $\begin{array}{l}21.6 \\
21\end{array}$ & $\begin{array}{l}0.249 \\
0.249\end{array}$ & $\begin{array}{l}0.0219 \\
0.0202\end{array}$ & $\begin{array}{l}0.06377 \\
0.0637\end{array}$ & $\begin{array}{l}1.7 \\
1.72\end{array}$ & ${ }_{5}^{4}$ & 42 & $\begin{array}{l}226 \\
224\end{array}$ & $\begin{array}{l}59 \\
42\end{array}$ & $\begin{array}{l}40 \\
43\end{array}$ & $\begin{array}{l}78 . \\
56\end{array}$ & & $\begin{array}{r}20 \\
25\end{array}$ & ${ }_{13}^{13}$ & & 3 & $\begin{array}{l}1.86 \\
.75\end{array}$ \\
\hline ii & 5 & 33 & 97.53 & $\begin{array}{l}96.02 \\
96.25\end{array}$ & $\begin{array}{l}0.41 \\
0.47\end{array}$ & 4.24 & 2.98 & 12.4 & 0.085 & $\begin{array}{l}0.44 \\
0.04\end{array}$ & $\begin{array}{l}0.8353 \\
0.928\end{array}$ & $\begin{array}{l}21 \\
19.9\end{array}$ & $\begin{array}{l}0.2299 \\
0.275\end{array}$ & $\begin{array}{l}0.0202 \\
0.0231\end{array}$ & $\begin{array}{l}.06037 \\
0.0636\end{array}$ & $\begin{array}{l}1.72 \\
1.86\end{array}$ & $\begin{array}{l}5 \\
6\end{array}$ & $\begin{array}{l}31 \\
25 \\
25\end{array}$ & $\begin{array}{l}224 \\
222\end{array}$ & $\begin{array}{l}42 \\
69\end{array}$ & $\begin{array}{l}43 \\
37\end{array}$ & $\begin{array}{l}564 \\
669\end{array}$ & $\begin{array}{l}330 \\
273\end{array}$ & ${ }_{23}^{25}$ & $\begin{array}{l}13 \\
13\end{array}$ & ${ }_{0}^{3}$ & 3 & 1.75 \\
\hline 11 & 5 & 128 & 98.48 & 96.85 & 0.47 & 3.81 & 3 & 13.1 & 0.074 & 0.475 & 0.959 & 19.1 & 0.288 & 0.0281 & 0.0636 & 1.96 & 7 & 19 & 221 & 36 & 37 & 焉 & $\begin{array}{l}273 \\
335\end{array}$ & $\begin{array}{l}23 \\
21\end{array}$ & 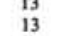 & $\begin{array}{l}0 \\
1\end{array}$ & $\begin{array}{l}3 \\
3\end{array}$ & $\begin{array}{l}1.7 \\
1.67\end{array}$ \\
\hline 11 & 6 & 1 & 98.71 & 97.08 & 0.55 & 3.83 & 3.05 & 13.4 & 0.072 & 0.455 & 0.967 & 19 & 0.297 & 0.0308 & 0.0635 & 1.96 & 8 & 22 & 220 & 34 & 39 & 595 & 322 & 25 & 13 & 0 & 3 & 1.45 \\
\hline 11 & 6 & 48 & 99.18 & 97.33 & 0.44 & 3.73 & 2.84 & 12.3 & 0.08 & 0.588 & 0.919 & 19.1 & 0.2 & 0.0289 & 0.0638 & 1.93 & 9 & 40 & 221 & 35 & 43 & 624 & 260 & 29 & 13 & 3 & 3 & 1.87 \\
\hline 11 & 6 & 73 & 99.43 & 97.58 & 0.4 & 3.51 & 2.84 & 12.3 & 0.079 & 0.708 & 0.934 & 18.6 & 0.277 & 0.0277 & 0.0637 & 1.99 & 7 & 49 & 221 & 36 & 45 & 578 & 347 & 25 & 13 & 1 & 3 & 1.73 \\
\hline 11 & 6 & $\begin{array}{r}98 \\
173\end{array}$ & 99.68 & $\begin{array}{l}97.83 \\
0808\end{array}$ & 0.41 & 3.33 & 2.85 & 12.5 & $\begin{array}{l}0.085 \\
0.075\end{array}$ & 0.75 & 0.942 & $\begin{array}{l}18.3 \\
188\end{array}$ & 0.291 & 0.0291 & 0.0637 & 2. & 8 & 57 & 221 & 44 & 44 & 570 & 275 & 20 & 13 & 1 & 3 & 1.72 \\
\hline 11 & 6 & 123 & 99.93 & $\begin{array}{l}98.08 \\
98.33\end{array}$ & $\begin{array}{l}0.42 \\
0.38\end{array}$ & 3.02 & $\begin{array}{l}2.88 \\
2.88\end{array}$ & 12.6 & 0.075 & 0.778 & 0.947 & $\begin{array}{l}18.7 \\
18.8\end{array}$ & 0.296 & 0.03 & 0.0638 & 2. & 7 & 58 & 2222 & 38 & 43 & 609 & 332 & 19 & 13 & 0 & 3 & 1.95 \\
\hline II & 6 & $\begin{array}{r}148 \\
23\end{array}$ & $\begin{array}{l}100.18 \\
100.43\end{array}$ & $\begin{array}{l}98.33 \\
98.43\end{array}$ & 0.38 & 3.1 & $\begin{array}{l}2.82 \\
2.94\end{array}$ & 12.4 & $\begin{array}{l}0.081 \\
0.076\end{array}$ & 0.662 & 0.931 & 18.8 & 0.286 & $\begin{array}{l}0.0256 \\
0.0262\end{array}$ & 0.0638 & 2.05 & $?$ & 53 & 222 & 41 & 43 & 616 & 373 & 23 & 13 & 0 & 3 & 1.96 \\
\hline $\begin{array}{l}11 \\
11 \\
11\end{array}$ & $?$ & $\begin{array}{l}23 \\
41\end{array}$ & $\begin{array}{l}100.43 \\
100.61\end{array}$ & $\begin{array}{l}98.43 \\
98.61\end{array}$ & 0.41 & 3.09 & 2.94 & 12.9 & 0.076 & 0.693 & 0.974 & 18.5 & 0.3 & 0.0262 & 0.0638 & 2.14 & 7 & 64 & 222 & 39 & 45 & 599 & 248 & 23. & 13 & 0 & 3 & 1.94 \\
\hline ii & CC & 13 & 100.65 & 98.65 & 0.5 & 3.44 & 3.09 & 13.5 & 0.075 & 0.502 & 0.984 & 18.9 & 0.306 & 0.0266 & 0.0635 & 2.09 & 8 & 44 & 220 & 36 & 39 & 597 & 330 & 23 & 13 & 0 & 3 & 1.51 \\
\hline 11 & CC & 38 & 100.9 & 98.9 & 0.42 & 3.12 & 2.88 & 12.5 & 0.091 & 0.641 & 0.932 & 19.1 & 0.283 & 0.0252 & 0.0636 & 2 & 7 & 42 & 222 & 39 & 46 & 648 & 373 & 19 & 13 & 2 & 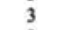 & 1.63 \\
\hline 12 & 1 & 23 & 100.93 & 100.93 & 0.27 & 2.1 & 2.48 & 10 & 0.076 & 0.565 & 0.826 & 22.2 & 0.237 & 0.016 & 0.0638 & 1.81 & 7 & 48 & 229 & 53 & 41 & 1465 & 262 & 19 & 14 & 0 & 3 & 94 \\
\hline 12 & 1 & 51 & 101.21 & 101.16 & 0.2 & 2.13 & 2.43 & 9.8 & 0.072 & 0.597 & 0.814 & 22.8 & 0.238 & 0.0152 & 0.0641 & 1.8 & 6 & 58 & 231 & 48 & 42 & 1415 & 159 & 38 & 14 & 0 & 3 & 2.36 \\
\hline 12 & 1 & 74 & 101.44 & $\begin{array}{l}101.39 \\
101\end{array}$ & $\begin{array}{l}0.39 \\
0.36\end{array}$ & 2.5 & 2.77 & 11.8 & 0.082 & 0.52 & 0.895 & 20.3 & 0.274 & 0.0238 & & 2.8 & 7 & 51 & 226 & 43 & 36 & 796 & 341 & 21 & 13 & 0 & 3 & 1.78 \\
\hline 12 & 1 & 101 & 101.71 & $\begin{array}{l}101.64 \\
10187\end{array}$ & $\begin{array}{l}0.36 \\
0.35\end{array}$ & $\begin{array}{l}2.51 \\
2.47\end{array}$ & $\begin{array}{l}2.77 \\
2.75\end{array}$ & $\begin{array}{l}11.7 \\
11.5\end{array}$ & $\begin{array}{l}0.076 \\
0.069\end{array}$ & $\begin{array}{l}0.536 \\
0.53\end{array}$ & $\begin{array}{l}0.909 \\
0.994\end{array}$ & $\begin{array}{r}20.6 \\
20.6\end{array}$ & $\begin{array}{l}0.279 \\
0.27\end{array}$ & 0.0214 & & 2.8 & 8 & 51 & 226 & 49 & 40 & 1034 & 289 & 21 & 13 & 0 & 3 & 1.72 \\
\hline${ }_{12}^{12}$ & 1 & $\begin{array}{l}124 \\
148\end{array}$ & $\begin{array}{l}101.94 \\
102.18\end{array}$ & $\begin{array}{l}101.87 \\
102.17\end{array}$ & $\begin{array}{l}0.35 \\
0.31\end{array}$ & $\begin{array}{l}2.47 \\
2.5\end{array}$ & $\begin{array}{l}2.75 \\
2.79\end{array}$ & $\begin{array}{l}11.5 \\
11.5\end{array}$ & $\begin{array}{l}0.069 \\
0.061\end{array}$ & $\begin{array}{l}0.53 \\
0.455\end{array}$ & $\begin{array}{l}0.9144 \\
0.929\end{array}$ & ${ }_{21}^{20.6}$ & $\begin{array}{l}0.27 \\
0.26\end{array}$ & $\begin{array}{l}0.0193 \\
0.0172\end{array}$ & $\begin{array}{l}0.0637 \\
0.0638\end{array}$ & $\begin{array}{l}2.0 \\
2.0\end{array}$ & $?$ & $\begin{array}{l}48 \\
47 \\
47\end{array}$ & $\begin{array}{l}227 \\
227\end{array}$ & 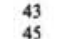 & $\begin{array}{l}39 \\
44\end{array}$ & & $\begin{array}{l}364 \\
327\end{array}$ & 22 & 13 & 0 & 3 & 1.81 \\
\hline $\begin{array}{l}12 \\
12\end{array}$ & $\begin{array}{l}1 \\
2\end{array}$ & $\begin{array}{r}148 \\
23\end{array}$ & $\begin{array}{l}102.48 \\
102.43\end{array}$ & $\begin{array}{l}102.11 \\
102.36\end{array}$ & $\begin{array}{l}0.31 \\
0.34\end{array}$ & $\begin{array}{l}2.5 \\
2.41\end{array}$ & 2.8 & $\begin{array}{l}11.5 \\
11.6\end{array}$ & $\begin{array}{l}0.061 \\
0.056\end{array}$ & $\begin{array}{l}0.455 \\
0.446\end{array}$ & $\begin{array}{l}0.929 \\
0.92\end{array}$ & $\begin{array}{l}21 \\
21.3\end{array}$ & $\begin{array}{l}0.26 \\
0.267\end{array}$ & $\begin{array}{l}0.0172 \\
0.017\end{array}$ & $\begin{array}{l}0.0638 \\
0.0638\end{array}$ & 2.0 & 9 & $\begin{array}{l}47 \\
35\end{array}$ & $\begin{array}{l}227 \\
227\end{array}$ & 45 & ${ }_{43}^{44}$ & 1139 & 332 & ${ }_{27}^{22}$ & 13 & 1 & 3 & 1.93 \\
\hline 12 & 2 & 51 & 102.71 & 102.63 & 0.32 & 2.58 & 2.83 & 11.6 & 0.061 & 0.505 & $\begin{array}{l}0.92 \\
0.941\end{array}$ & $\begin{array}{l}21.3 \\
20.8\end{array}$ & 0.265 & 0.0169 & $\begin{array}{l}0.0638 \\
0.0638\end{array}$ & $\begin{array}{l}1.99 \\
2.05\end{array}$ & 9 & $\begin{array}{l}35 \\
52\end{array}$ & $\begin{array}{l}227 \\
226\end{array}$ & $\begin{array}{l}51 \\
58 \\
58\end{array}$ & ${ }_{45}^{43}$ & 112 & $\begin{array}{l}332 \\
342\end{array}$ & $\begin{array}{l}37 \\
37 \\
37\end{array}$ & $\begin{array}{l}13 \\
13\end{array}$ & 2 & ${ }_{3}^{3}$ & 1.9 \\
\hline 12 & 2 & 73 & 102.93 & 102.85 & 0.3 & 2.25 & 2.64 & 10.8 & 0.052 & 0.485 & 0.884 & 21.8 & 0.25 & 0.0154 & 0.0636 & $\begin{array}{l}1.92 \\
1.92\end{array}$ & 7 & 44 & 228 & $\begin{array}{l}38 \\
49\end{array}$ & $\begin{array}{l}43 \\
39\end{array}$ & 131 & $\begin{array}{l}342 \\
309\end{array}$ & $\begin{array}{l}37 \\
26\end{array}$ & $\begin{array}{l}13 \\
13\end{array}$ & 0 & $\begin{array}{l}3 \\
3\end{array}$ & $\begin{array}{l}1.9 \\
1.7\end{array}$ \\
\hline 23 & 1 & 25 & 207.15 & 207.15 & 0.05 & 1.63 & 1.5 & 6 & 0.152 & 0.736 & 0.473 & 27.6 & 0.132 & 0.0096 & 0.0642 & 1.04 & 3 & 45 & 239 & 82 & 37 & 1370 & 318 & 22 & 14 & 2 & 3 & 2.53 \\
\hline 23 & 1 & 50 & 207.4 & 207.4 & 0.04 & 1.74 & 1.7 & 6.8 & 0.1 & 0.716 & 0.562 & 26 & 0.153 & 0.0 & & 1.2 & 1 & 47 & 237 & 63 & 43 & 130 & 366 & 20 & 14 & 0 & 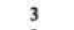 & 2.4 \\
\hline 23 & 2 & 31 & 208.71 & 207.63 & 0.12 & 1.8 & 1.72 & 7.1 & 0.203 & 0.70 & 0.555 & 26 & 0.164 & & & 1. & 4 & 49 & 236 & 73 & 43 & 13 & 32 & 23 & 14 & 0 & 3 & 3. \\
\hline 23 & 2 & 58 & 208.98 & 207.9 & 0.09 & 1.81 & 1.7 & $\begin{array}{l}7.3 \\
0\end{array}$ & $\begin{array}{l}0.229 \\
0.232\end{array}$ & 0.7 & & $\begin{array}{l}25.3 \\
200\end{array}$ & & 0.0119 & & & 3 & $\begin{array}{l}56 \\
75\end{array}$ & 23 & 63 & 47 & & & 22 & 14 & 0 & 3 & 33 \\
\hline${ }_{23}^{23}$ & 2 & $\begin{array}{r}83 \\
106\end{array}$ & $\begin{array}{r}209.23 \\
209.46\end{array}$ & ${ }_{208}^{208}$ & 0.13 & $\begin{array}{l}2.12 \\
2\end{array}$ & $\begin{array}{l}1.87 \\
1.64\end{array}$ & $\begin{array}{l}9 \\
81\end{array}$ & 0.2 & $\begin{array}{l}0.939 \\
0.802\end{array}$ & $\begin{array}{l}0.602 \\
0.515\end{array}$ & $\frac{22.9}{25}$ & 0.1 & 0.0 & & t. & 5 & 75 & 23 & 78 & 48 & & $2 t$ & 21 & 14 & 0 & 3 & 55 \\
\hline $\begin{array}{l}23 \\
23 \\
23\end{array}$ & $\begin{array}{l}2 \\
2\end{array}$ & $\begin{array}{l}106 \\
131\end{array}$ & $\begin{array}{l}2099.46 \\
209.71\end{array}$ & $\begin{array}{l}208.38 \\
208.63\end{array}$ & $\begin{array}{l}0.1 \\
0.15\end{array}$ & $\begin{array}{l}2 \\
2.32\end{array}$ & $\begin{array}{l}1.64 \\
1.85\end{array}$ & $\begin{array}{r}8.1 \\
11.1\end{array}$ & $\begin{array}{l}0.23 \\
0.199\end{array}$ & $\begin{array}{l}0.802 \\
0.919\end{array}$ & $\begin{array}{l}0.5515 \\
0.588\end{array}$ & $\begin{array}{l}25 \\
21.5\end{array}$ & $\begin{array}{l}0.167 \\
0.19\end{array}$ & $\begin{array}{l}0.0108 \\
0.0172\end{array}$ & $\begin{array}{l}0.0641 \\
0.0643\end{array}$ & 1. & $\begin{array}{l}3 \\
4\end{array}$ & ${ }_{74}^{44}$ & ${ }_{22}^{23}$ & 59 & 43 & & 14 & 23 & 14 & ${ }_{0}^{0}$ & 3 & 14 \\
\hline 23 & 3 & 6 & 209.96 & 208.88 & 0.14 & 2.01 & 1.81 & 8.1 & 0.181 & $\begin{array}{l}0.919 \\
0.85\end{array}$ & $\begin{array}{l}0.588 \\
0.59\end{array}$ & 24 & $\begin{array}{l}0.19 \\
0.186\end{array}$ & $\begin{array}{l}0.0172 \\
0.0154\end{array}$ & $\begin{array}{l}0.0643 \\
0.0642\end{array}$ & $\begin{array}{l}1.54 \\
1.51\end{array}$ & $\begin{array}{l}4 \\
5\end{array}$ & $\begin{array}{l}74 \\
63\end{array}$ & $\begin{array}{l}228 \\
234\end{array}$ & $\begin{array}{l}59 \\
59\end{array}$ & 43 & & 14 & 21 21 & $\begin{array}{l}13 \\
14\end{array}$ & 0 & ${ }^{3}$ & \\
\hline 23 & 3 & 31 & 210.21 & 209.13 & 0.14 & 1.99 & 2 & 8.3 & 0.105 & 0.704 & 0.653 & 23.8 & 0.201 & $\begin{array}{l}0.0154 \\
0.0153\end{array}$ & $\begin{array}{l}0.042 \\
0.0639\end{array}$ & 1.68 & 3 & $\begin{array}{l}83 \\
\text { so }\end{array}$ & $\begin{array}{l}234 \\
233\end{array}$ & $\begin{array}{l}39 \\
46\end{array}$ & $\begin{array}{l}41 \\
40\end{array}$ & 124 & 22 & $\begin{array}{l}21 \\
25\end{array}$ & $\begin{array}{l}14 \\
14\end{array}$ & $\begin{array}{l}0 \\
0\end{array}$ & $\begin{array}{l}3 \\
3\end{array}$ & 18 \\
\hline 23 & 3 & 56 & 210.46 & 209.38 & 0.13 & 1.74 & 1.85 & 7.7 & 0.114 & 0.736 & 0.602 & 24.6 & 0.189 & 0.0155 & 0.06 & 1.5 & 3 & 59 & 235 & so & 40 & 1263 & 253 & 21 & 14 & 0 & 3 & 29 \\
\hline 23 & 3 & 81 & 210.71 & 209.63 & 0.19 & 1.86 & 2 & 8.1 & 0.105 & 0.7 & 0.653 & 24.3 & 0.1 & 0.0 & & 1. & 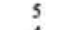 & 60 & 233 & 53 & 41 & 124 & 28 & 19 & 14 & 0 & 3 & 9 \\
\hline 23 & 3 & 106 & 210.96 & 209 & 0.13 & 1.8 & 1.87 & 7.7 & 0.104 & 0.6 & & 25 & 0. & & & & 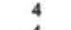 & 48 & 23. & 59 & 38 & 12 & 2 & 23 & 14 & 0 & 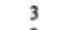 & ?7 \\
\hline $\begin{aligned} 23 \\
23\end{aligned}$ & 3 & 131 & $\begin{array}{r}211.21 \\
211.41\end{array}$ & 210 & 0.1 & 2.0 & 2.1 & 8.6 & 0.1 & 0.6 & & $\begin{array}{l}24.1 \\
23.6\end{array}$ & & & & & 4 & 56 & 23 & 57 & 39 & & 22 & 20 & 14 & 0 & 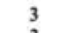 & 24 \\
\hline $\begin{aligned} 23 \\
23\end{aligned}$ & 4 & $\begin{array}{r}6 \\
39\end{array}$ & $\begin{array}{l}211.46 \\
211.79\end{array}$ & 211 & 0. & $\begin{array}{r}2.04 \\
2.24\end{array}$ & 2. & 8.6 & & & & ${ }_{23}^{23}$ & & & & & $\begin{array}{l}4 \\
5\end{array}$ & 50 & 23 & & 41 & & 2 & 19 & 14 & 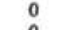 & 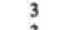 & 4 \\
\hline $\begin{array}{l}23 \\
23 \\
23\end{array}$ & $\begin{array}{l}4 \\
4\end{array}$ & $\begin{array}{l}39 \\
64\end{array}$ & $\begin{array}{l}2111.79 \\
212.04\end{array}$ & $\begin{array}{l}210.6 \\
210.85\end{array}$ & $\begin{array}{l}0.22 \\
0.22\end{array}$ & $\begin{array}{l}2.24 \\
2.34\end{array}$ & $\begin{array}{l}2.27 \\
2.2\end{array}$ & $\begin{array}{l}9 \\
8.6\end{array}$ & 0.101 & $\begin{array}{l}0.695 \\
0.794\end{array}$ & & $\begin{array}{l}23.4 \\
23.7\end{array}$ & & & & & $\begin{array}{l}5 \\
5\end{array}$ & 56 & ${ }_{23}^{23}$ & 56 & ${ }_{39}^{43}$ & & & 21 & 14 & 0 & 3 & \\
\hline 23 & 4 & $\begin{array}{l}84 \\
89\end{array}$ & 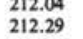 & $\begin{array}{l}210.85 \\
211.1\end{array}$ & $\begin{array}{l}0.22 \\
0.22\end{array}$ & $\begin{array}{l}2.34 \\
2.36\end{array}$ & $\begin{array}{l}2.2 \\
2.24\end{array}$ & $\begin{array}{l}8.6 \\
8.8\end{array}$ & $\begin{array}{l}0.11 \\
0.123\end{array}$ & $\begin{array}{l}0.724 \\
0.72\end{array}$ & $\begin{array}{l}0.723 \\
0.723\end{array}$ & $\begin{array}{l}23.7 \\
23.1\end{array}$ & $\begin{array}{l}0.195 \\
0.203\end{array}$ & $\begin{array}{l}.0137 \\
0.0155\end{array}$ & & 1.64 & 5 & $\begin{array}{l}37 \\
56\end{array}$ & $\begin{array}{l}232 \\
231\end{array}$ & $\begin{array}{l}80 \\
55 \\
\end{array}$ & $\begin{array}{l}39 \\
42\end{array}$ & 112 & $\begin{array}{l}10 \\
29\end{array}$ & $\begin{array}{l}24 \\
20\end{array}$ & 14 & $\begin{array}{l}0 \\
0\end{array}$ & $\begin{array}{l}3 \\
3\end{array}$ & $\begin{array}{l}04 \\
21\end{array}$ \\
\hline 23 & 4 & 123 & 212 & & 0.22 & 2.14 & 2.01 & 7.9 & 0.1 & 0.8 & & 24.3 & 0. & & & . & 3 & 54 & 23 & 54 & 45 & i17 & 371 & 21 & 14 & 0 & 3 & 16 \\
\hline 23 & 5 & 14 & 213.04 & & 0.3 & 2. & 2.59 & 10.3 & 0.2 & & & 20 & & & & 2. & 1 & 86 & 228 & 55 & 51 & 9 & 28 & 37 & 13 & 0 & 3 & 1 \\
\hline 23 & 5 & 35 & 213 & & 0.2 & 2.2 & 2.54 & 10.5 & 0. & & & 20 & & & & & ? & *⿻一𠃋火 & 22 & 93 & 52 & & 28 & 22 & 14 & 1 & 3 & 5 \\
\hline 23 & 5 & 92 & 213 & & 0.3 & 2. & 2.43 & 10.2 & 0.1 & & & 20 & & & & & 8 & 110 & 22 & 9 & sc & & & 19 & 14 & 0 & 3 & \\
\hline 23 & 5 & 117 & 214 & & 0.1 & 2. & 2.12 & 8.9 & & 0. & 0. & 22 & & & & & 6 & 89 & 2 & 73 & 48 & & 12 & 21 & 14 & 0 & 3 & \\
\hline${ }_{23}^{23}$ & 5 & 142 & 214 & $\begin{array}{l}212 \\
212\end{array}$ & 0.1 & 2. & 2. & 8.7 & 0.1 & & & 22 & & & & & $?$ & 9. & 2 & & & & & 23 & 1 & 0 & 3 & 6 \\
\hline $\begin{array}{l}23 \\
23\end{array}$ & $\begin{array}{l}6 \\
6\end{array}$ & $\begin{array}{l}17 \\
39\end{array}$ & $\begin{array}{l}214.57 \\
214.79\end{array}$ & $\begin{array}{l}212 \\
213\end{array}$ & $\begin{array}{l}0.1 \\
0.0\end{array}$ & $\begin{array}{l}1.9 \\
1.7\end{array}$ & 2. & $\begin{array}{l}8.3 \\
6.8\end{array}$ & & & & $\begin{array}{l}23 \\
26\end{array}$ & & & & & $\begin{array}{l}3 \\
4 \\
4\end{array}$ & 8. & & $\begin{array}{l}7 \\
8\end{array}$ & 48 & & & 20 & 14 & 0 & 3 & \\
\hline $\begin{array}{l}23 \\
23\end{array}$ & $\begin{array}{l}6 \\
6\end{array}$ & $\begin{array}{l}39 \\
64\end{array}$ & $\begin{array}{l}214.79 \\
215.04\end{array}$ & $\begin{array}{l}213 \\
213\end{array}$ & $\begin{array}{l}0.0 \\
0.0\end{array}$ & $\begin{array}{l}1.72 \\
1.67\end{array}$ & $\begin{array}{l}1.6 \\
1.5\end{array}$ & $\begin{array}{l}6.8 \\
6.1\end{array}$ & $\begin{array}{l}0 . \\
0 .\end{array}$ & & . & $\begin{array}{l}26 \\
26\end{array}$ & & & & & ${ }_{3}^{4}$ & $\begin{array}{l}71 \\
74\end{array}$ & 2 & 8. & 48 & & & 22 & $\begin{array}{l}14 \\
14\end{array}$ & 0 & 3 & \\
\hline 23 & 6 & $\begin{array}{l}54 \\
89\end{array}$ & $\begin{array}{l}215 \\
215\end{array}$ & & 0. & i. & 1.65 & $\begin{array}{l}6.1 \\
6.7\end{array}$ & & & & $\begin{array}{l}25.1 \\
25.8\end{array}$ & & & & i. & 5 & 73 & $\begin{array}{l}23, \\
23\end{array}$ & 86 & $\begin{array}{l}31 \\
49\end{array}$ & 128 & 20 & $\begin{array}{l}22 \\
26\end{array}$ & 14 & $\begin{array}{l}0 \\
0\end{array}$ & 3 & $\begin{array}{l}2.53 \\
2.26\end{array}$ \\
\hline 23 & 6 & 110 & & & 0.12 & 1.79 & 1.83 & 7.5 & 0.1 & & 0.6 & 25.1 & 0.1 & & & 1. & 4 & 6 & 23 & 7 & 46 & 124 & 29 & 28 & 14 & 0 & 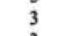 & 32 \\
\hline 23 & 7 & 15 & & & 0.19 & 1.9 & 1.9 & 8.2 & 0.149 & 0.737 & 0.627 & 24 & 0.192 & 0.015 & 0.0639 & 1.55 & 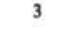 & 45 & 233 & 54 & 44 & 1123 & 161 & 20 & 14 & 0 & 3 & 2.14 \\
\hline $\begin{array}{l}23 \\
23 \\
23\end{array}$ & $\stackrel{7}{C C}$ & 41 & $\begin{array}{l}216 \\
216\end{array}$ & & & & & & & & & & & & & & 4 & 56 & & & & & 29 & & & 0 & 3 & \\
\hline $\begin{array}{l}23 \\
24\end{array}$ & $\begin{array}{c}\mathrm{CC} \\
1\end{array}$ & $\begin{array}{l}21 \\
25 \\
25\end{array}$ & $\begin{array}{l}216 . \\
216 .\end{array}$ & $\begin{array}{l}214.6 \\
216.75\end{array}$ & $\begin{array}{l}0 . \\
0 .\end{array}$ & $\begin{array}{l}2.18 \\
2.22\end{array}$ & 2. & 10 & & & 0.801 & 21 & & & & & 7 & $\begin{array}{l}30 \\
87\end{array}$ & & $\begin{array}{l}36 \\
62\end{array}$ & 48 & & 29 & ${ }_{27}^{23}$ & $\begin{array}{l}14 \\
14\end{array}$ & 0 & $\begin{array}{l}3 \\
3\end{array}$ & \\
\hline 24 & 1 & so & 217 & 217 & 0.2 & 2.23 & 2.42 & 9.8 & & & & 21.3 & & & & & 8 & 10 & 22 & $\infty$ & 52 & & 29 & 20 & 14 & 0 & 3 & \\
\hline 4 & 1 & 74 & 217.24 & 217.24 & 0.3 & 2.36 & 2.8 & 11.4 & & & & $\begin{array}{l}19.4 \\
\end{array}$ & & & & 2. & 9 & 106 & 22 & $\infty$ & 53 & 6 & 28 & 21 & $\begin{array}{l}14 \\
13\end{array}$ & 0 & 3 & \\
\hline 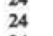 & i & 115 & 217.65 & 217.5 & 0.3 & 2.12 & 2.78 & 12.5 & & & & 17.9 & & & & 2. & 9 & 159 & 22 & 97 & 58 & 75 & 28 & 22 & $\begin{array}{l}15 \\
13\end{array}$ & 0 & 3 & 9 \\
\hline 24 & i & 137 & & & 0.3 & 2.49 & 3.03 & 12.5 & & & & 19 & & & & & 10 & 8 & 22 & 60 & so & 83 & 28 & 21 & 13 & 0 & 3 & 96 \\
\hline 24 & 2 & is & 218.15 & 218 & 0.28 & 1.9 & 2.58 & 10.5 & & & & 20 & & & & & 9 & 10 & 22 & 8. & 49 & & 78 & 20 & 14 & 0 & 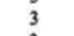 & 25 \\
\hline 24 & 2 & 37.5 & $\begin{array}{l}218.375 \\
\end{array}$ & $\begin{array}{r}218.23 \\
2\end{array}$ & 0.2 & 1.64 & 2.18 & 9.1 & 0.145 & 0.97 & & 22.2 & 0.2 & & & 1. & 6 & 10 & 232 & 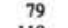 & 47 & 107 & 172 & 20 & 14 & 0 & 3 & 41 \\
\hline 24 & 2 & 63 & 218.63 & 218.48 & & 0.94 & 1.46 & 23.8 & 0.088 & 1.076 & 0.443 & 12.7 & 0.163 & 0.0133 & 0.0654 & 1.51 & 8 & 90 & 211 & 119 & 54 & 733 & 242 & 18 & 14 & 13 & 3 & 4.35 \\
\hline
\end{tabular}




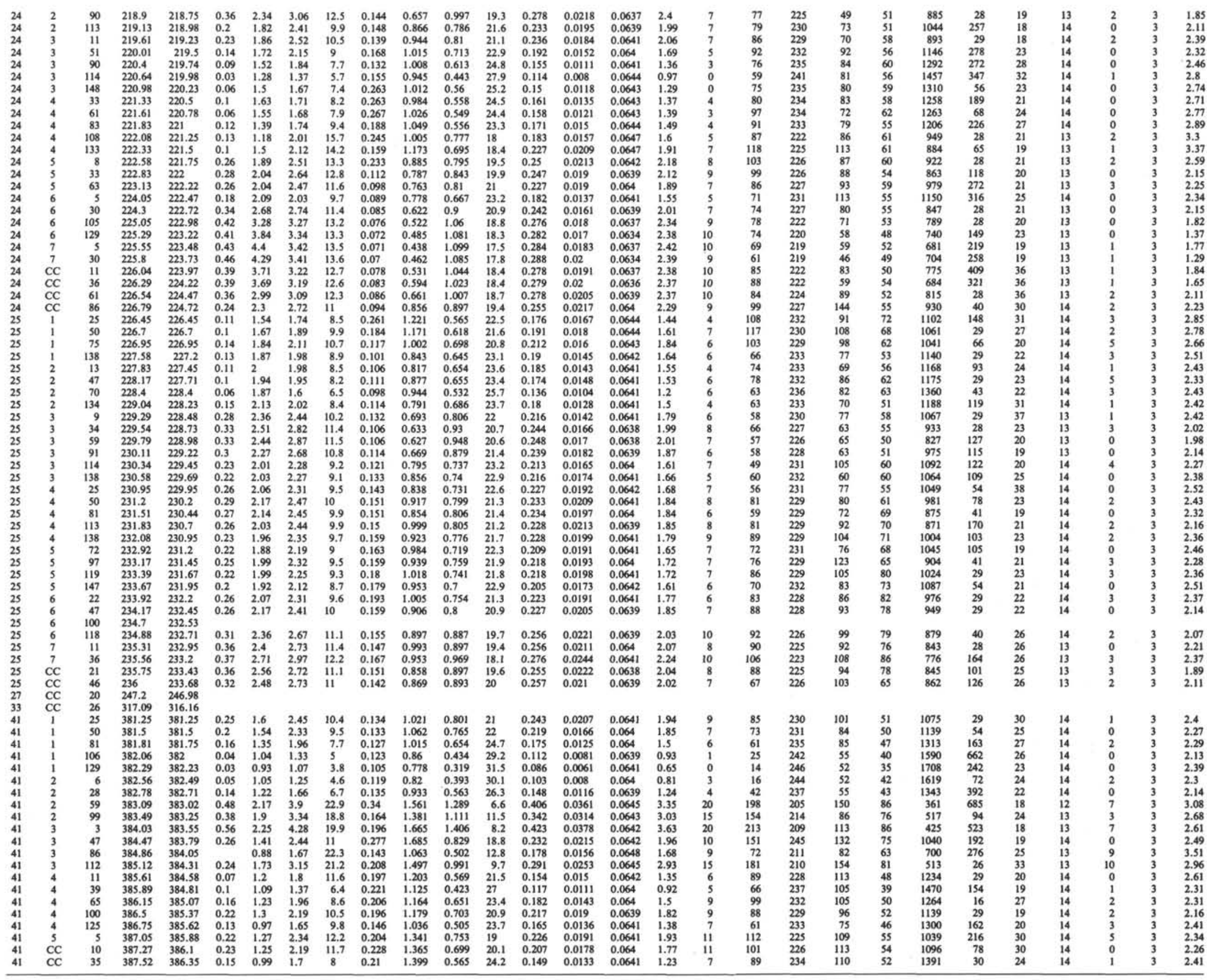


Table 2. Hole 728B XRF data. * indicates unreliable trace element abundances.

\begin{tabular}{|c|c|c|c|c|c|c|c|c|c|c|c|c|c|c|c|c|c|c|c|c|c|c|c|c|c|c|c|c|}
\hline $\begin{array}{l}\text { Core } \\
\text { no. }\end{array}$ & $\begin{array}{l}\text { Sec. } \\
\text { no. }\end{array}$ & $\begin{array}{l}\text { Sec. } \\
\text { depth } \\
(\mathrm{cm})\end{array}$ & $\begin{array}{l}\text { ODP } \\
\text { depth } \\
\text { (mbsf) }\end{array}$ & $\begin{array}{l}\text { True } \\
\text { depth } \\
\text { (mbsf) }\end{array}$ & $\begin{array}{c}\mathrm{Na} \\
(\%)\end{array}$ & $\begin{array}{l}\mathrm{Mg}_{(\%)} \\
(\%)\end{array}$ & $\begin{array}{c}\mathrm{Al} \\
(\%)\end{array}$ & $\underset{(\%)}{S i}$ & $\underset{(\%)}{P}$ & $\underset{(\%)}{\mathbf{S}}$ & $\underset{(\%)}{\mathbf{K}}$ & $\begin{array}{c}\mathrm{Ca} \\
(\%)\end{array}$ & $\begin{array}{c}\mathrm{Ti} \\
(\%)\end{array}$ & $\begin{array}{c}\mathrm{Cr} \\
(\%)\end{array}$ & $\begin{array}{l}\mathrm{Mn} \\
(\%)\end{array}$ & $\begin{array}{c}\mathrm{Fe} \\
(\%)\end{array}$ & $\underset{\text { (ppm) }}{\mathrm{Co}}$ & $\underset{(\mathrm{ppm})}{\mathrm{Ni}}$ & $\underset{(\mathrm{ppm})}{\mathrm{Cu}}$ & $\underset{(\mathrm{ppm})}{\mathrm{Zn}}$ & $\begin{array}{c}\mathrm{Rb} \\
(\mathrm{ppm})\end{array}$ & $\underset{(\mathrm{ppm})}{\mathrm{Sr}}$ & $\begin{array}{c}\mathrm{Ba} \\
(\mathrm{ppm})\end{array}$ & $\underset{(\mathrm{ppm})}{\mathrm{La}}$ & $\begin{array}{c}\mathrm{Pb} \\
(\mathrm{ppm})\end{array}$ & $\underset{(\mathrm{ppm})}{\mathrm{Th}}$ & $\underset{(\mathrm{ppm})}{\mathrm{U}}$ & $\begin{array}{l}\text { SALT } \\
(\%)\end{array}$ \\
\hline 20 & 4 & 25 & 178.55 & 178.55 & 0.01 & 1.81 & 2.20 & 11.50 & 0.043 & 0.555 & 0.727 & 21.5 & 0.192 & 0.0119 & 0.0640 & 1.82 & 11 & 33 & 229 & 63 & 40 & 1373 & 29 & 25 & 14 & 2 & 3 & 2.26 \\
\hline 20 & 4 & 50 & 178.8 & 178.8 & & 1.45 & 1.60 & 8.69 & 0.059 & 0.410 & 0.520 & 25.2 & 0.155 & 0.0122 & 0.0638 & 1.30 & 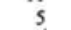 & 12 & 235 & 55 & 31 & 1517 & 176 & 39 & 14 & 0 & 3 & 2.01 \\
\hline 20 & 4 & 75 & 179.05 & 179.05 & 0.01 & 1.66 & 1.83 & 8.85 & 0.072 & 0.585 & 0.611 & 24.2 & 0.168 & 0.0116 & 0.0639 & 1.50 & 8 & 30 & 233 & 72 & 35 & 1535 & 30 & 27 & 14 & 1 & 3 & 2.03 \\
\hline 20 & 4 & 97 & 179.27 & 179.27 & 0.05 & 1.83 & 2.07 & 9.40 & 0.068 & 0.693 & 0.699 & 22.3 & 0.204 & 0.0143 & 0.0640 & 1.83 & 10 & 59 & 231 & 82 & 47 & 1233 & 79 & 21 & 14 & 3 & 3 & 2.19 \\
\hline 20 & 4 & 125 & 179.55 & 179.55 & 0.13 & 2.19 & 2.59 & 11.50 & 0.064 & 0.719 & 0.884 & 19.2 & 0.249 & 0.0208 & 0.0638 & 2.29 & 12 & 77 & 27 & 88 & 58 & 1189 & 154 & 22 & 14 & 1 & 3 & 2 \\
\hline 20 & 4 & 150 & 179.8 & 179.8 & 0.14 & 2.27 & 2.77 & 11.94 & 0.065 & 0.611 & 0.946 & 19.1 & 0.259 & 0.0198 & 0.0639 & 2.41 & 13 & 79 & 227 & 88 & 58 & 1205 & 672 & 26 & 13 & 3 & 3 & 2.03 \\
\hline 20 & 5 & 25 & 180.05 & 180.05 & 0.13 & 2.12 & 2.74 & 12.45 & 0.057 & 0.682 & 0.929 & 18.8 & 0.253 & 0.0182 & 0.0638 & 2.39 & 14 & 72 & 225 & 82 & 55 & 1195 & 88 & 19 & 13 & 2 & 3 & 1.94 \\
\hline 20 & 5 & so & 180.3 & 180.3 & 0.03 & 1.93 & 2.37 & 11.30 & 0.061 & 0.626 & 0.791 & 21.1 & 0.209 & 0.0111 & 0.0639 & 1.99 & 9 & 53 & 229 & 76 & 46 & 1410 & 334 & 19 & 14 & 0 & 3 & 2.14 \\
\hline 20 & 5 & 75 & 180.55 & 180.55 & & 1.39 & 1.48 & 7.45 & 0.060 & 0.496 & 0.474 & 26.9 & 0.120 & 0.0068 & 0.0641 & 1.09 & 8 & 12 & 238 & 68 & 32 & 1753 & 83 & 26 & 14 & 5 & 3 & 2.36 \\
\hline 20 & 5 & 97 & 180.77 & 180.77 & & 1.39 & 1.59 & 7.91 & 0.067 & 0.494 & 0.516 & 26.1 & 0.133 & 0.0078 & 0.0640 & 1.23 & 6 & ${ }_{21}$ & 236 & 72 & 32 & 1691 & 30 & 32 & 14 & 0 & 3 & 2.23 \\
\hline 20 & 5 & 125 & 181.05 & 181.05 & 0.02 & 1.65 & 1.92 & 8.79 & 0.081 & 0.609 & 0.653 & 24.4 & 0.164 & 0.0106 & 0.0640 & 1.56 & 7 & 38 & 234 & 83 & 40 & 1619 & 30 & 38 & 14 & 1 & 3 & 2.18 \\
\hline 20 & 5 & 148 & 181.28 & 181.28 & 0.04 & 1.86 & 2.13 & 10.17 & 0.080 & 0.661 & 0.723 & 22.0 & 0.191 & 0.0137 & 0.0639 & 1.85 & 10 & 60 & 230 & 80 & 44 & 1473 & 568 & 25 & 14 & 4 & 3 & 2.15 \\
\hline 20 & 6 & 25 & 181.55 & 181.55 & 0.14 & 2.07 & 2.48 & 12.10 & 0.079 & 0.693 & 0.839 & 19.3 & 0.237 & 0.0174 & 0.0639 & 2.18 & 13 & 76 & 226 & 77 & 46 & 1258 & 552 & 26 & 14 & 3 & 3 & 2.04 \\
\hline 20 & 6 & & 181.8 & 181.8 & 0.17 & 2.24 & 2.74 & 13.53 & 0.068 & 0.687 & 0.927 & 17.7 & 0.260 & 0.0215 & 0.0638 & 2.46 & 13 & 80 & 223 & 71 & 46 & 1125 & 810 & 36 & 13 & 2 & 3 & 1.92 \\
\hline 20 & 6 & 75 & 182.05 & 182.05 & 0.21 & 2.37 & 3.02 & 13.99 & 0.053 & 0.721 & 1.023 & 16.6 & 0.281 & 0.0223 & 0.0637 & 2.65 & 13 & 88 & 222 & 68 & 50 & 1038 & 811 & 24 & 13 & 4 & 3 & 1.77 \\
\hline 20 & 6 & 100 & 182.3 & 182.3 & 0.18 & 2.25 & 2.94 & 14.12 & 0.056 & 0.617 & 0.969 & 17.3 & 0.270 & 0.0196 & 0.0639 & 2.49 & 12 & 69 & 223 & 74 & 48 & 1170 & 847 & 21 & 13 & 1 & 3 & 2.13 \\
\hline 20 & 6 & 125 & 182.55 & 182.55 & 0.12 & 2.15 & 2.73 & 13.67 & 0.055 & 0.626 & 0.899 & 18.5 & 0.233 & 0.0162 & 0.0637 & 2.26 & 13 & 60 & 223 & 78 & 46 & 1213 & 501 & 36 & 13 & 5 & 3 & 1.83 \\
\hline 20 & 6 & 150 & 182.8 & 182.8 & & 1.53 & 1.51 & 9.62 & 0.068 & 0.483 & 0.474 & 25.2 & 0.130 & 0.0087 & 0.0638 & 1.15 & 5 & 12 & 233 & 64 & 30 & 1584 & 30 & 39 & 14 & 3 & 3 & 1.97 \\
\hline 27 & 5 & 25 & 247.75 & 247.75 & 0.07 & 1.66 & 1.81 & 8.31 & 0.046 & 0.387 & 0.599 & 25.7 & 0.151 & 0.0080 & 0.0636 & 1.38 & 8 & 20 & 235 & 71 & 31 & 1742 & 29 & 21 & 14 & 1 & 3 & 1.66 \\
\hline 27 & 5 & 52 & 248.02 & 248.02 & & 1.38 & 1.56 & 8.39 & 0.054 & 0.393 & 0.501 & 26.3 & 0.133 & 0.0070 & 0.0638 & 1.21 & 4 & 12 & 236 & 60 & 28 & 1779 & 30 & 25 & 14 & 0 & 3 & 1.93 \\
\hline 27 & 5 & 75 & 248.25 & 248.25 & 0.04 & 1.60 & 1.82 & 9.16 & 0.069 & 0.437 & 0.608 & 24.6 & 0.155 & 0.0088 & 0.0638 & 1.40 & 7 & 42 & 234 & 81 & 33 & 1681 & 513 & 23 & 14 & 0 & 3 & 1.95 \\
\hline 27 & 5 & 100 & 248.5 & 248.5 & 0.07 & & 2.06 & 9.91 & 0.073 & 0.486 & 0.710 & 22.7 & 0.186 & 0.0116 & 0.0637 & 1.71 & 7 & 46 & 230 & 84 & 38 & 1569 & 29 & 21 & 14 & 5 & 3 & 1.77 \\
\hline 27 & 5 & 125 & 248.75 & 248.75 & 0.10 & 1.97 & 2.39 & 10.68 & 0.070 & 0.534 & 0.842 & 20.8 & 0.230 & 0.0164 & 0.0637 & 2.02 & 8 & 62 & 228 & 78 & 41 & 1388 & 29 & 22 & 14 & 2 & 3 & 1.79 \\
\hline 27 & 5 & 149 & 248.99 & 248.99 & 0.29 & 2.28 & 2.84 & 12.49 & 0.069 & 0.552 & 0.990 & 18.9 & 0.283 & 0.0213 & 0.0635 & 2.49 & 10 & 89 & 225 & 75 & 50 & 1230 & 1099 & 31 & 13 & 2 & 3 & 1.49 \\
\hline 27 & 6 & & 249.25 & 249.25 & 0.25 & 2.45 & 3.22 & 13.60 & 0.063 & 0.586 & 1.107 & 16.4 & 0.307 & 0.0214 & 0.0636 & 2.77 & 14 & 95 & 222 & 85 & 51 & 1097 & 840 & 21 & 13 & 1 & 3 & 1.67 \\
\hline 27 & 6 & 50 & 249.5 & 249.5 & 0.26 & 2.40 & 3.28 & 13.71 & 0.058 & 0.644 & 1.127 & 16.9 & 0.301 & 0.0211 & 0.0636 & 2.91 & 13 & 87 & 223 & 82 & 54 & 1120 & 829 & 21 & 13 & i & 3 & 1.58 \\
\hline 27 & 6 & 75 & 249.75 & 249.75 & 0.07 & 1.72 & 2.18 & 9.08 & 0.051 & 0.460 & 0.753 & 24.0 & 0.171 & 0.0084 & 0.0637 & 1.64 & 9 & 44 & 232 & 80 & 41 & 1697 & 29 & 20 & 14 & 5 & 3 & 1.83 \\
\hline 27 & 6 & 100 & 250 & 250 & 0.00 & 1.44 & 1.75 & 7.81 & 0.057 & 0.402 & 0.584 & 26.3 & 0.140 & 0.0079 & 0.0638 & 1.28 & 5 & 32 & 237 & 71 & 31 & 1805 & 308 & 23 & 14 & 0 & 3 & 1.94 \\
\hline 27 & 6 & 125 & 250.25 & 250.25 & 0.08 & 1.80 & 2.26 & 9.66 & 0.067 & 0.442 & 0.794 & 22.6 & 0.199 & 0.0150 & 0.0637 & 1.75 & 8 & 42 & 23 & 87 & 38 & 1592 & 616 & 38 & 14 & 1 & 3 & 1.82 \\
\hline 27 & 6 & 150 & 250.5 & 250.5 & 0.33 & 2.66 & 3.46 & 14.71 & 0.083 & 0.665 & 1.167 & 15.3 & 0.321 & 0.0287 & 0.0635 & 2.98 & 15 & 126 & 21 & 99 & 57 & 1050 & 823 & 35 & 13 & 4 & 3 & 1.46 \\
\hline 27 & 7 & 25 & 250.75 & 250.75 & 0.39 & 2.92 & 3.97 & 16.52 & 0.069 & 0.750 & 1.325 & 13.5 & 0.356 & 0.0300 & 0.0634 & 3.22 & 18 & 123 & 21 & 96 & 58 & 746 & 781 & 22 & 13 & 1 & 3 & 1.38 \\
\hline 27 & $\mathrm{CC}$ & 4 & 250.95 & 250.95 & 0.20 & 2.16 & 2.95 & 12.44 & 0.051 & 0.468 & 1.002 & 19.1 & 0.254 & 0.0168 & 0.0635 & 2.33 & 10 & 51 & 22 & 71 & 47 & 1309 & 821 & 22 & 13 & 3 & 3 & 1.5 \\
\hline 27 & CC & 30 & 251.2 & 251.2 & 0.15 & 2.09 & 2.26 & 10.09 & 0.052 & 0.355 & 0.731 & 23.0 & 0.186 & 0.0128 & 0.0635 & 1.72 & 10 & 37 & 22 & 58 & 37 & 1355 & 288 & 20 & 14 & 6 & 3 & 1.41 \\
\hline 28 & 1 & 4 & 251.24 & 251.24 & 0.08 & 1.65 & 1.87 & 9.45 & 0.062 & 0.381 & 0.617 & 24.4 & 0.161 & 0.0094 & 0.0635 & 1.46 & 7 & 29 & 23 & 70 & 34 & 1688 & 67 & 23 & 14 & 2 & 3 & 1.41 \\
\hline 28 & 1 & 26 & 251.46 & 251.46 & 0.06 & 1.60 & 1.63 & 8.37 & 0.061 & 0.327 & 0.518 & 26.0 & 0.142 & 0.0093 & 0.0636 & 1.22 & 5 & 25 & 23 & 68 & 26 & 1760 & 55 & 31 & 14 & 0 & 3 & 1.58 \\
\hline 28 & 1 & 51 & 251.71 & 251.71 & 0.06 & 1.73 & 1.75 & 8.28 & 0.064 & 0.304 & 0.569 & 25.5 & 0.152 & 0.0098 & 0.0635 & 1.34 & 9 & 24 & 23 & 67 & 31 & 1697 & 137 & 22 & 14 & 3 & 3 & 1.45 \\
\hline 28 & 1 & 76 & 251.96 & 251.96 & 0.09 & 2.08 & 2.18 & 9.83 & 0.080 & 0.519 & 0.738 & 22.1 & 0.205 & 0.0147 & 0.0636 & 1.78 & 8 & 56 & 22 & 83 & 38 & 1495 & 28 & 18 & 14 & 4 & 3 & 1.7 \\
\hline 28 & 1 & 104 & 252.24 & 252.24 & 0.23 & 2.39 & 2.73 & 12.41 & 0.086 & 0.595 & 0.914 & 18.5 & 0.265 & 0.0221 & 0.0636 & 2.41 & 11 & 85 & 22 & 90 & 46 & 1230 & 540 & 18 & 13 & $i$ & 3 & 1.56 \\
\hline 28 & 1 & 126 & 252.46 & 252.46 & 0.21 & 2.45 & 2.69 & 12.08 & 0.075 & 0.554 & 0.910 & 18.9 & 0.256 & 0.0222 & 0.0636 & 2.32 & ii & 72 & 22 & 85 & 42 & 1261 & 676 & 28 & 13 & 2 & 3 & 1.62 \\
\hline 28 & 2 & 4 & 252.74 & 252.74 & 0.25 & 2.30 & 2.51 & 11.42 & 0.069 & 0.518 & 0.850 & 20.9 & 0.249 & 0.0164 & 0.0635 & 2.13 & 9 & 94 & 22 & 82 & 44 & 1327 & 981 & 31 & 13 & 3 & 3 & 1.47 \\
\hline 28 & 2 & 26 & 252.96 & 252.96 & 0.21 & 2.27 & 2.50 & 11.79 & 0.077 & 0.512 & 0.843 & 20.8 & 0.248 & 0.0182 & 0.0637 & 2.0 & 8 & 82 & 22 & 79 & 45 & 1410 & 832 & 32 & 13 & 3 & 3 & 1.77 \\
\hline 28 & 2 & 51 & 253.21 & 253.21 & 0.25 & 2.23 & 2.49 & 11.74 & 0.070 & 0.461 & & 21.5 & 0.242 & 0.0151 & 0.0636 & 2.0 & 8 & 69 & 22 & 68 & 39 & 1421 & 89 & 33 & 13 & 2 & 3 & 1.63 \\
\hline 28 & 2 & 76 & 253.46 & 253 & 0.23 & 2.23 & 2.66 & 11.88 & 0.062 & 0.453 & & 20.7 & 0.2 & 0.0 & 0.0635 & 2.2 & 10 & 84 & 22 & 75 & 43 & 1356 & 1170 & 31 & 13 & 1 & 3 & 1.55 \\
\hline 28 & 2 & 104 & 253.74 & 253 & 0.25 & 2.25 & 2.71 & 12. & 0.0 & 0.439 & & 20.3 & 0.2 & & 0.0636 & 2.2 & 9 & 84 & 22 & 79 & 45 & 1344 & 642 & 32 & 13 & 4 & 3 & 1.68 \\
\hline 28 & 2 & 126 & 253.96 & & 0.19 & 1.97 & 2.30 & 10. & & & & 23.4 & & & & 1.7 & 9 & 75 & & 82 & 43 & 15 & 26 & 32 & 13 & 7 & 3 & 1.5 \\
\hline 28 & 3 & 4 & 254 & 254 & 0.21 & 2.14 & 2.52 & 12. & & & & 20.9 & & & & 2. & 9 & 77 & & 83 & 39 & 138 & 48 & 32 & 13 & 1 & 3 & 1.61 \\
\hline 28 & 3 & 26 & 254 & & 0.27 & 2.51 & 3.19 & 15.2 & 0.0 & & & 16.8 & & & & 2. & 11 & 100 & & 88 & 50 & 11 & 118 & 31 & 13 & 4 & 3 & 1.69 \\
\hline 28 & 3 & 51 & & & 0.23 & 2.38 & 2.98 & 14. & & & & 18.5 & & & & & 11 & 104 & & 86 & 48 & 128 & 105 & 32 & 13 & 2 & 3 & 1.65 \\
\hline 28 & 3 & 76 & & & 0.12 & 1.64 & 1.53 & 8. & & & & 28.0 & & & & & 3 & 25 & & 46 & 24 & 17 & 70 & 34 & 14 & 1 & 3 & 1.52 \\
\hline 28 & 3 & 101 & & & 0. & 1. & 1.78 & 9. & & & & 26.0 & & & & & 5 & 4 & & 68 & 30 & & 4 & 34 & 14 & 1 & 3 & 1.82 \\
\hline 28 & 3 & 126 & & & 0. & 2. & 2.40 & & & & & & & & & & 9 & 10 & & 83 & 40 & 142 & 18 & 33 & 13 & 2 & 3 & 1.55 \\
\hline 28 & 4 & 1 & & & 0. & 3. & & & & & & 14 & & & & 2. & 14 & 15 & & 97 & 50 & 81 & 104 & 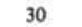 & 13 & 2 & 3 & 1.41 \\
\hline 28 & 4 & 26 & & & 0. & & & 16. & & & & & & & & & 15 & 159 & & 133 & 54 & & 9. & $3 c$ & 13 & 3 & 3 & 1.52 \\
\hline 28 & 4 & 51 & & & & & & 6. & & & & & & & & & 2 & 26 & & G & 23 & & 104 & 35 & 14 & 2 & 3 & 1.64 \\
\hline 28 & 4 & 76 & & & & & & & & & & 28 & & & & & 0 & 12 & & 27 & 22 & 18 & 45 & 33 & 14 & 1 & 3 & 1.43 \\
\hline 28 & 4 & 101 & & & 0. & 2. & & & & & & 26 & & & & & 2 & 24 & & 9 & 26 & & 13 & 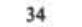 & 14 & 0 & 3 & 1.75 \\
\hline 28 & 4 & 126 & & & 0. & 2. & 2. & 12. & & & & & & & & & 7 & 71 & & 73 & 33 & 14 & & 32 & 13 & 0 & 3 & 1.68 \\
\hline 28 & 5 & 1 & & & 0. & 2. & & & & & & & & & & & 8 & 79 & & 7 & 40 & 13 & 11 ? & 31 & 13 & 0 & 3 & 1.57 \\
\hline 28 & 5 & 26 & & & 0. & 2. & & 13. & & & & & & & & & 9 & 91 & 2 & 73 & 43 & & 10 & 31 & 13 & 0 & 3 & 1.41 \\
\hline 28 & 5 & 51 & & & 0. & 1. & 1. & 8. & & & & & & & & & 3 & 14 & & 5 & 19 & & 100 & 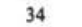 & 14 & 0 & 3 & 1.65 \\
\hline 28 & 5 & 7 & & & & 1. & 1. & & & & & & & & & & 6 & 54 & & 6 & 28 & & 77 & 33 & 14 & 2 & 3 & 1.37 \\
\hline 28 & 5 & 10 & & & & 1. & 1. & & & & & & & & & & 3 & 35 & 2 & 6 & 23 & & 71 & 3. & 14 & 0 & 3 & 1.52 \\
\hline 28 & 5 & 126 & & & 0. & & 2. & & & & & & & & & & 7 & 8 & 22 & 75 & 37 & 13 & 12 & 31 & 13 & 0 & 3 & 1.48 \\
\hline 28 & 6 & 1 & & & 0. & 2. & 3. & 14. & & & & & & & & & 14 & 135 & 22 & 91 & 46 & 11 & 117 & $3 c$ & 13 & 1 & 3 & 1.39 \\
\hline 28 & 6 & 26 & & & 0. & 1.2 & 1. & 6. & & & & & & & & & 4 & 38 & & 65 & 18 & 19 & 94 & 35 & 14 & 0 & 3 & 1.49 \\
\hline 28 & 6 & 51 & & & 0. & 1.4 & 1.5 & 8. & & & & 28 & & & & & 3 & 44 & 23 & 67 & 25 & 18 & 85 & 34 & 14 & 0 & 3 & 1.77 \\
\hline 28 & 6 & 76 & 259.46 & 259.46 & 0.19 & 1.85 & 2.17 & 10.35 & 0.080 & 0.503 & 0.726 & 23.4 & 0.213 & 0.0136 & 0.0635 & 1.82 & 8 & 83 & 232 & 81 & 34 & 1545 & 981 & 33 & 14 & 0 & 3 & 1.55 \\
\hline 28 & 6 & 101 & 259.71 & 259.71 & 0.18 & 1.82 & 2.11 & 10.52 & 0.077 & 0.484 & 0.706 & 23.4 & 0.205 & 0.0129 & 0.0636 & 1.76 & 6 & 72 & 232 & 70 & 29 & 1532 & 847 & 33 & 14 & 0 & 3 & 1.57 \\
\hline
\end{tabular}




\begin{tabular}{|c|c|c|c|c|c|c|c|c|c|c|c|c|c|c|c|c|c|c|c|c|c|c|c|c|c|c|}
\hline 28 & 6 & 126 & 259.96 & 259.96 & 0.09 & 1.38 & 1.53 & 69 & 0.057 & 0.394 & 0.492 & 26.6 & 0.138 & 0.0052 & 0.0636 & 1.20 & 3 & 39 & 235 & 60 & 24 & 1754 & 916 & 34 & 14 & 0 \\
\hline 28 & 7 & 1 & 260.21 & 260.21 & 0.21 & 2.23 & 2.69 & 12.52 & 0.048 & 0.402 & 0.905 & 20.5 & 0.242 & 0.0161 & 0.0635 & 2.13 & 10 & 81 & 225 & 72 & 39 & 1335 & 223 & 31 & 13 & 1 \\
\hline 28 & 7 & 26 & 260.46 & 260.46 & 0.12 & 1.71 & 1.78 & 8.52 & 0.060 & 0.472 & 0.584 & 25.8 & 0.173 & 0.0102 & 0.0637 & 1.44 & 4 & 51 & 235 & 70 & 23 & 1662 & 1040 & 34 & 14 & 0 \\
\hline 28 & CC & 9 & 260.69 & 260.69 & 0.27 & 2.13 & 2.38 & 11.76 & 0.063 & 0.549 & 0.795 & 21.1 & 0.236 & 0.0165 & 0.0635 & 2.03 & 10 & 95 & 227 & 85 & 38 & 1345 & 914 & 31 & 13 & 5 \\
\hline 29 & 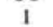 & 10 & 260.9 & 260.9 & 0.24 & 2.15 & 2.58 & 13.02 & 0.101 & 0.480 & 0.881 & 20.3 & 0.253 & 0.0175 & 0.0636 & 2.05 & 8 & 76 & 225 & 80 & 57 & 1383 & 247 & 32 & 27 & 8 \\
\hline & 1 & 35 & 261.15 & 261.15 & 0.34 & 2.32 & 2.98 & 14.62 & 0.100 & 0.521 & 1.005 & 17.6 & 0.295 & 0.0209 & 0.0635 & 2.41 & 9 & 81 & 221 & 77 & 74 & 1225 & 1209 & 31 & 46 & 16 \\
\hline & i & 60 & 261.4 & 261.4 & 0.35 & 2.46 & 3.29 & 15.79 & 0.092 & 0.588 & 1.117 & 16.2 & 0.323 & 0.0219 & 0.0635 & 2.68 & 11 & 98 & 219 & 82 & 359 & 1156 & 1100 & 30 & 176 & 45 \\
\hline & i & 85 & 261.65 & 261.65 & 0.25 & 2.34 & 3.14 & 15.36 & 0.073 & 0.517 & 1.048 & 17.7 & 0.281 & 0.0173 & 0.0636 & 2.49 & ii & 100 & 222 & 80 & 358 & 1241 & 897 & 32 & 175 & 44 \\
\hline & i & 110 & 261.9 & 261.9 & 0.15 & 1.52 & 1.72 & 9.51 & 0.066 & 0.397 & 0.572 & 26.1 & 0.157 & 0.0076 & 0.0635 & 1.35 & 3 & 41 & 235 & 58 & 360 & 1582 & 989 & 34 & 177 & 44 \\
\hline & i & 135 & 262.15 & 262.15 & 0.13 & 1.42 & 1.50 & 8.85 & 0.063 & 0.328 & 0.466 & 27.6 & 0.143 & 0.0077 & 0.0636 & 1.14 & 4 & 39 & 237 & 55 & 359 & 1652 & 593 & 34 & 177 & 44 \\
\hline & 2 & 10 & 262.4 & 262.4 & 0.18 & 1.91 & 1.90 & 9.62 & 0.073 & 0.474 & 0.617 & 25.0 & 0.181 & 0.0106 & 0.0636 & 1.51 & 5 & 56 & 233 & 69 & 360 & 1544 & 42 & 33 & 177 & 44 \\
\hline & 2 & 35 & 262.65 & 262.65 & 0.26 & 2.43 & 2.35 & 11.75 & 0.088 & 0.620 & 0.772 & 20.8 & 0.239 & 0.0181 & 0.0637 & 2.07 & 7 & 93 & 227 & 84 & 358 & 1337 & 585 & 33 & 179 & 45 \\
\hline & 2 & 60 & 262.9 & 262.9 & 0.38 & 2.88 & 3.19 & 15.48 & 0.082 & 0.782 & 1.045 & 16.0 & 0.313 & 0.0249 & 0.0636 & 2.96 & 13 & 133 & 220 & 98 & 357 & 1045 & 364 & 30 & 176 & 45 \\
\hline & 2 & 85 & 263.15 & 263.15 & 0.42 & 2.80 & 3.47 & 16.65 & 0.068 & 0.705 & 1.150 & 14.9 & 0.327 & 0.0260 & 0.0635 & 3.09 & 13 & 141 & 217 & 89 & 355 & 1005 & 623 & 30 & 174 & 44 \\
\hline & 2 & 110 & 263.4 & 263.4 & 0.30 & 2.49 & 3.12 & 15.10 & 0.064 & 0.539 & 1.035 & 17.4 & 0.286 & 0.0203 & 0.0637 & 2.58 & 11 & 100 & 222 & 81 & 357 & 1224 & 753 & 31 & 175 & 44 \\
\hline & 2 & 135 & 263.65 & 263.65 & 0.15 & $\begin{array}{l}1.48 \\
1.38\end{array}$ & $\begin{array}{l}3.12 \\
1.50\end{array}$ & 9.10 & $\begin{array}{l}0.084 \\
0.084\end{array}$ & $\begin{array}{l}0.339 \\
0.375\end{array}$ & 0.454 & 27.5 & $\begin{array}{l}0.1480 \\
0.143\end{array}$ & 0.0078 & 0.0638 & 1.09 & 4 & 33 & 237 & 59 & 357 & 1650 & 921 & 34 & 175 & 43 \\
\hline & 3 & 10 & 263.9 & 263.9 & 0.19 & $\begin{array}{l}1.49 \\
\end{array}$ & 1.65 & 9.26 & 0.098 & 0.471 & $\begin{array}{l}0.456 \\
0.560\end{array}$ & 25.0 & 0.140 & 0.0134 & 0.0637 & 1.28 & 50 & 221 & 318 & 210 & 17 & 1583 & 136 & 31 & 14 & 0 \\
\hline & 3 & 35 & 264.15 & 264.15 & 0.19 & $\begin{array}{l}1.83 \\
1.83\end{array}$ & 2.23 & 12.66 & 0.085 & 0.487 & 0.760 & 21.7 & 0.210 & 0.0117 & 0.0637 & 1.76 & 5 & 67 & 227 & 73 & 357 & 1455 & 201 & 32 & 175 & 44 \\
\hline & 3 & 60 & 264.4 & 264.4 & 0.11 & $\begin{array}{l}1.53 \\
1.57\end{array}$ & 1.82 & $\begin{array}{l}10.90 \\
10.92\end{array}$ & 0.096 & 0.434 & 0.595 & 24.7 & $\begin{array}{l}0.172 \\
0\end{array}$ & 0.0095 & 0.0638 & 1.39 & 3 & 35 & 233 & 59 & 357 & 1593 & 451 & 34 & 176 & 43 \\
\hline & 3 & 85 & 264.65 & 264.65 & 0.15 & 1.44 & 1.66 & 9.83 & 0.095 & 0.420 & 0.544 & 25.8 & 0.173 & 0.0091 & 0.0637 & 1.27 & 4 & 32 & 234 & 69 & 358 & 1590 & 925 & 34 & 176 & 43 \\
\hline & 3 & 110 & 264.9 & 264.9 & 0.21 & 1.81 & 2.14 & $\begin{array}{r}71.00 \\
11.02\end{array}$ & 0.072 & 0.442 & 0.719 & 23.4 & 0.217 & 0.0132 & 0.0637 & $\begin{array}{l}1.71 \\
1.70\end{array}$ & 7 & 68 & 230 & 73 & 359 & 1523 & 272 & 33 & 175 & 43 \\
\hline & 3 & 135 & 265.15 & 265.15 & 0.23 & 2.04 & 2.46 & 11.48 & 0.064 & 0.484 & 0.842 & 21.4 & 0.249 & 0.0184 & 0.0637 & 2.06 & 8 & 77 & 229 & 71 & 362 & $\begin{array}{l}1469 \\
\end{array}$ & 1244 & 32 & 176 & 44 \\
\hline 29 & 4 & 10 & 265.4 & 265.4 & 0.32 & 2.19 & 2.81 & 12.95 & 0.062 & 0.555 & 0.947 & $\begin{array}{l}19.6 \\
\end{array}$ & 0.270 & 0.0198 & 0.0635 & 2.41 & 10 & 88 & 225 & 73 & 360 & 1324 & 827 & 31 & 175 & 44 \\
\hline 29 & 4 & 35 & 265.65 & 265.65 & 0.26 & 2.00 & 2.58 & 12.21 & 0.063 & 0.493 & 0.882 & 21.4 & 0.238 & 0.0140 & 0.0636 & 2.04 & 9 & 73 & 228 & 73 & 359 & 1418 & 164 & 33 & 175 & 44 \\
\hline 29 & 4 & 60 & 265.9 & 265.9 & 0.19 & 1.67 & 1.94 & 10.18 & 0.088 & 0.399 & 0.644 & 25.1 & 0.184 & 0.0135 & 0.0637 & 1.48 & 5 & 43 & 233 & 67 & 358 & 1556 & 387 & 34 & 176 & 43 \\
\hline 29 & 4 & 85 & 266.15 & $266.15^{\circ}$ & & 1.87 & 2.16 & 10.00 & 0.091 & 0.520 & 0.765 & 22.4 & 0.187 & 0.0183 & 0.0637 & 1.79 & 63 & 253 & 327 & 223 & 17 & 1348 & 28 & 20 & 14 & 0 \\
\hline 29 & 4 & 110 & 266.4 & $266.4^{\circ}$ & 0.07 & 2.46 & 2.99 & $\begin{array}{l}13.63 \\
\end{array}$ & 0.088 & 0.579 & 1.052 & 17.1 & 0.275 & 0.0280 & 0.0635 & 2.53 & 65 & 291 & 308 & 221 & 17 & 1028 & 343 & 18 & 13 & 0 \\
\hline 29 & 4 & 135 & 266.65 & $266.65^{*}$ & 0.21 & 2.89 & 3.80 & 16.68 & 0.064 & 0.511 & 1.327 & 13.8 & 0.333 & 0.0326 & 0.0634 & 3.05 & 65 & 292 & 289 & 224 & 16 & 679 & 26 & 22 & 13 & 0 \\
\hline 29 & 5 & 10 & 266.9 & $266.9^{\circ}$ & & 1.81 & 2.23 & 11.77 & 0.079 & 0.473 & 0.793 & 21.4 & 0.200 & 0.0215 & 0.0635 & 1.77 & 63 & 244 & 303 & 210 & 17 & 1325 & 28 & 19 & 14 & 0 \\
\hline 29 & 5 & 35 & 267.15 & $267.155^{\circ}$ & 0.02 & 2.16 & 2.64 & 13.10 & 0.081 & 0.472 & 0.928 & 19.4 & 0.237 & 0.0243 & 0.0634 & 2.20 & 64 & 278 & 322 & 221 & 17 & 1137 & 27 & 19 & 13 & 0 \\
\hline 29 & 5 & 60 & 267.4 & $267.4^{\circ}$ & 0.08 & 2.40 & 2.85 & 14.09 & 0.092 & 0.655 & 0.990 & 17.1 & 0.264 & 0.0279 & 0.0635 & 2.50 & 65 & 308 & 324 & 243 & 17 & 1032 & 27 & 36 & 13 & 0 \\
\hline 29 & 5 & 85 & 267.65 & $267.65^{*}$ & 0.19 & 2.71 & 3.37 & 17.13 & 0.061 & 0.633 & 1.148 & 14.0 & 0.315 & 0.0309 & 0.0018 & 3.00 & 67 & 320 & 306 & 232 & 16 & 507 & 26 & 33 & 13 & 0 \\
\hline 29 & 5 & 110 & 267.9 & $267.9^{\circ}$ & & 2.11 & 2.68 & 13.59 & 0.051 & 0.444 & 0.944 & 18.7 & 0.233 & 0.0203 & 0.0634 & 2.22 & 64 & 249 & 294 & 214 & 17 & 1142 & 27 & 22 & 13 & 0 \\
\hline 29 & 5 & 135 & 268.15 & $268.15^{\circ}$ & & 2.02 & 2.25 & 12.92 & 0.101 & 0.397 & 0.758 & 20.7 & 0.203 & 0.0241 & 0.0635 & 1.76 & 62 & 241 & 311 & 214 & 17 & 1279 & 27 & 22 & 13 & 0 \\
\hline 29 & 6 & 10 & 268.4 & $268.4^{\circ}$ & & 2.11 & 2.30 & 12.15 & 0.097 & 0.524 & 0.790 & 19.8 & 0.221 & 0.0229 & 0.0635 & 2.01 & 62 & 266 & 306 & 210 & 17 & 1229 & 27 & 29 & 14 & 0 \\
\hline 29 & 6 & 35 & 268.65 & $268.65^{*}$ & & 2.25 & 2.47 & 12.59 & 0.085 & 0.515 & 0.854 & 19.2 & 0.230 & 0.0236 & & 2.15 & 63 & 268 & 308 & 216 & 17 & 1178 & 27 & 29 & 14 & 0 \\
\hline 29 & 6 & 60 & 268.9 & $268.9^{\circ}$ & & 2.05 & 2.20 & 11.63 & 0.072 & 0.425 & 0.756 & 21.0 & 0.198 & 0.0218 & 0.0635 & 1.86 & 64 & 249 & 307 & 208 & 17 & 1332 & 41 & 37 & 14 & 0 \\
\hline 29 & 6 & 85 & 269.15 & $269.15^{*}$ & & 1.70 & 1.61 & 8.79 & 0.069 & 0.332 & 0.539 & 25.4 & 0.128 & 0.0140 & 0.0634 & 1.36 & 63 & 222 & 309 & 203 & 17 & 1564 & 29 & 39 & 14 & 0 \\
\hline 29 & 6 & 110 & 269.4 & $269.4^{\circ}$ & & 2.25 & 2.12 & 10.93 & 0.074 & 0.466 & 0.728 & 21.3 & 0.205 & 0.0232 & 0.0635 & 1.83 & 62 & 256 & 305 & 213 & 17 & 1357 & 28 & 37 & 14 & 0 \\
\hline 29 & 6 & 135 & 269.65 & $269.65^{\circ}$ & 0.07 & 2.54 & 2.75 & 13.33 & 0.063 & 0.486 & 0.938 & 18.1 & 0.248 & 0.0272 & 0.0635 & 2.36 & 64 & 265 & 303 & 215 & 17 & 1179 & 542 & 25 & 13 & 0 \\
\hline 29 & 7 & 10 & 269.9 & $269.9^{\circ}$ & 0.09 & 2.58 & 3.22 & 14.61 & 0.066 & 0.531 & 1.119 & 16.4 & 0.277 & 0.0255 & 0.0635 & 2.65 & 65 & 281 & 292 & 226 & 17 & 1037 & 26 & 21 & 13 & 0 \\
\hline 29 & 7 & 35 & 270.15 & $270.15^{*}$ & & 1.45 & 1.59 & 8.47 & 0.064 & 0.408 & 0.561 & 25.3 & 0.131 & 0.0139 & 0.0636 & 1.30 & 61 & 217 & 309 & 208 & 17 & 1587 & 29 & 21 & 14 & 0 \\
\hline 29 & CC & 16 & 270.37 & $270.37^{\circ}$ & & 1.64 & 1.80 & 9.49 & 0.057 & 0.401 & 0.646 & 23.8 & 0.159 & 0.0167 & 0.0634 & 1.51 & 61 & 213 & 297 & 198 & 17 & 1609 & 28 & 20 & 14 & 0 \\
\hline 29 & CC & 41 & 270.62 & $270.62^{*}$ & & 1.84 & 1.98 & 9.28 & 0.045 & 0.411 & 0.715 & 23.2 & 0.184 & 0.0174 & 0.0633 & 1.79 & 65 & 247 & 309 & 199 & 17 & 1428 & 352 & 30 & 14 & 0 \\
\hline 30 & 1 & 10 & 270.6 & $270.6^{\circ}$ & & 1.46 & 1.31 & 7.67 & 0.054 & 0.291 & 0.432 & 27.4 & 0.099 & 0.0109 & 0.0634 & 1.00 & 61 & 187 & 292 & 182 & 18 & 1563 & 56 & 21 & 14 & 0 \\
\hline 30 & i & 35 & 270.85 & $270.85^{*}$ & & 1.89 & 1.89 & 9.35 & 0.055 & 0.376 & 0.664 & 23.7 & 0.163 & 0.0190 & 0.0633 & 1.58 & 62 & 225 & 304 & 216 & 17 & 1438 & 28 & 18 & 14 & 0 \\
\hline 30 & i & 60 & 271.1 & $271.1^{\circ}$ & & 2.25 & 2.21 & 10.98 & 0.061 & 0.490 & 0.776 & 21.1 & 0.210 & 0.0229 & 0.0636 & 1.92 & 62 & 245 & 309 & 237 & 17 & 1428 & 28 & 19 & 14 & 0 \\
\hline 30 & i & 85 & 271.35 & $271.35^{*}$ & 0.11 & 2.53 & 3.05 & 14.47 & 0.052 & 0.581 & 1.061 & 16.4 & 0.283 & 0.0316 & 0.0633 & 2.68 & 65 & 276 & 297 & 216 & 17 & 1008 & 230 & 34 & 13 & 0 \\
\hline 30 & $i$ & 110 & 271.6 & $271.6^{\circ}$ & & 1.82 & 1.95 & 10.63 & 0.080 & 0.394 & 0.675 & 22.6 & 0.193 & 0.0209 & 0.0634 & 1.57 & 60 & 216 & 309 & 197 & 17 & 139 & 388 & 24 & 14 & 0 \\
\hline 30 & 1 & 135 & 271.85 & $271.85^{*}$ & & 2.00 & 2.22 & 12.00 & 0.071 & 0.505 & 0.779 & 20.1 & 0.228 & 0.0286 & 0.0635 & 1.94 & 62 & 241 & 299 & 210 & 17 & 127 & 27 & 23 & 14 & 0 \\
\hline 30 & 2 & 10 & 272.1 & $272.1^{*}$ & 0.05 & 2.38 & 2.89 & 14.51 & 0.048 & 0.545 & 1.019 & 16.6 & 0.275 & 0.0294 & 0.0634 & 2.54 & 64 & 276 & 290 & 214 & 17 & 98 & 26 & 34 & 13 & 0 \\
\hline 30 & 2 & 35 & 272.35 & $272.35^{*}$ & & 1.51 & 1.54 & 9.67 & 0.062 & 0.317 & 0.521 & 25.0 & 0.140 & 0.0170 & 0.0635 & 1.21 & 60 & 201 & 301 & 189 & 17 & 154 & 321 & 23 & 14 & 0 \\
\hline 30 & 2 & 60 & 272.6 & $272.6^{\circ}$ & & 1.78 & 1.91 & 10.04 & 0.066 & 0.460 & 0.667 & 22.9 & 0.179 & 0.0195 & 0.0635 & 1.66 & 62 & 232 & 314 & 210 & 17 & 143 & 260 & 23 & 14 & 0 \\
\hline 30 & 2 & 85 & 272.85 & $272.85^{*}$ & 0.12 & 2.41 & 2.76 & 15.31 & 0.064 & 0.688 & 0.945 & 16.1 & 0.279 & 0.0298 & 0.0636 & 2.61 & 65 & 300 & 310 & 231 & 17 & 95 & 26 & 35 & 13 & 0 \\
\hline 30 & 2 & 110 & 273.1 & $273.1^{*}$ & 0.08 & 2.58 & 3.14 & 16.08 & 0.043 & 0.553 & 1.087 & 15.6 & 0.273 & 0.0263 & 0.0634 & 2.72 & 66 & 285 & 30 & 216 & 16 & 9 & 26 & 3. & 13 & 0 \\
\hline 30 & 2 & 135 & 273.35 & $273.35^{*}$ & & 1.74 & 1.78 & 10.58 & 0.067 & 0.416 & 0.621 & 23.2 & 0.166 & 0.0157 & 0.0635 & 1.47 & 61 & 220 & 310 & 199 & 17 & 14 & 28 & 3 & 14 & 0 \\
\hline 30 & 3 & 10 & 273.6 & $273.6^{\circ}$ & & 1.90 & 2.12 & 11.93 & 0.054 & 0.471 & 0.768 & 20.5 & 0.203 & 0.0215 & 0.0635 & 1.83 & 62 & 23 & 29 & 203 & 17 & 12 & 28 & 3 & 14 & 0 \\
\hline 30 & 3 & 35 & 273.85 & $273.85^{\circ}$ & & 2.03 & 2.15 & 11.77 & 0.058 & 0.435 & 0.757 & 20.9 & 0.201 & 0.0196 & 0.0635 & 1.8 & 62 & 23 & 31 & 208 & 17 & 12 & 28 & 15 & 14 & 0 \\
\hline 30 & 3 & 60 & 274.1 & $274.1^{\circ}$ & 0.02 & 2.15 & 2.28 & 12.04 & 0.053 & 0.428 & 0.777 & 20.2 & 0.204 & 0.0203 & 0.0635 & 1.89 & 62 & 229 & 29 & 2 & 17 & 12 & 27 & 15 & 14 & c \\
\hline 30 & 3 & 85 & 274.35 & $274.35^{*}$ & & 1.89 & 2.00 & 10.63 & 0.053 & 0.388 & 0.707 & 22.5 & 0.166 & 0.0147 & 0.0633 & 1.67 & 63 & 22 & 31 & 2 & 17 & 14 & 28 & 37 & 14 & 0 \\
\hline 30 & 3 & 110 & 274.6 & $274.6^{\circ}$ & & 1.64 & 1.64 & 11.21 & 0.066 & 0.324 & 0.552 & 23.6 & 0.142 & 0.0129 & & 1.2 & 59 & 19 & 29 & 192 & 17 & 14 & 224 & 2 & 14 & c \\
\hline 30 & 3 & 135 & 274.85 & $274.85^{\circ}$ & & 1.80 & 1.92 & 12.19 & 0.066 & 0.403 & 0.658 & 21.9 & 0.178 & 0.0167 & 0.0637 & 1.62 & 61 & 22 & 30 & 201 & 17 & 14 & 29 & 2 & 14 & 0 \\
\hline 30 & 4 & 10 & 275.1 & $275.1^{*}$ & & 1.82 & 2.10 & 13.20 & 0.052 & 0.457 & 0.738 & 19.8 & 0.200 & 0.0199 & 0.0635 & 1.94 & 63 & 245 & 29 & 2 & 17 & 12 & 27 & 2 & 14 & c \\
\hline 30 & 4 & 35 & 275.35 & $275.35^{\circ}$ & 0.01 & 2.05 & 2.57 & 15.68 & 0.035 & 0.477 & 0.895 & 17.0 & 0.241 & 0.0232 & 0.0635 & 2.35 & 66 & 256 & 30 & 210 & 17 & 105 & 26 & 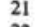 & 13 & c \\
\hline 30 & 4 & 60 & 275.6 & $275.6^{\circ}$ & & 1.86 & 2.21 & 12.94 & 0.034 & 0.448 & 0.778 & 20.2 & 0.192 & 0.0174 & 0.0634 & 1.96 & 64 & 241 & 29 & 199 & 17 & 13. & 41 & 2 & 14 & 0 \\
\hline 30 & 4 & 85 & 275.85 & $275.85^{*}$ & & 1.65 & 1.62 & 9.95 & 0.044 & 0.363 & 0.544 & 24.5 & 0.139 & 0.0125 & 0.0638 & 1.32 & 61 & 191 & 28 & 181 & 17 & 155 & 332 & 3 & 14 & c \\
\hline 30 & 4 & 110 & 276.1 & $276.10^{\circ}$ & & 1.47 & 1.50 & 9.60 & 0.050 & 0.334 & 0.512 & 24.8 & 0.133 & 0.0128 & 0.0635 & 1.35 & 60 & 195 & 302 & 188 & 17 & 1614 & 119 & 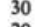 & 14 & c \\
\hline 30 & 4 & 135 & 276.35 & $276.35^{\circ}$ & & 2.05 & 2.27 & 12.05 & 0.058 & 0.543 & 0.797 & 20.0 & 0.229 & 0.0239 & 0.0635 & 2.06 & 64 & 258 & 30 & 219 & 17 & 1270 & 27 & 2 & 14 & c \\
\hline 30 & 5 & 10 & 276.6 & $276.6^{\circ}$ & 0.24 & 2.68 & 3.30 & 17.01 & 0.045 & 0.629 & 1.122 & 14.0 & 0.322 & 0.0342 & 0.0636 & 2.96 & 67 & 295 & 306 & 226 & 16 & 856 & 530 & 22 & 13 & c \\
\hline 30 & 5 & 35 & 276.85 & $276.85^{\circ}$ & 0.07 & 1.44 & 1.72 & 9.21 & 0.056 & 0.378 & 0.600 & 24.6 & 0.157 & 0.0110 & 0.0636 & 1.36 & 6 & 12 & 233 & 62 & 36 & 1646 & 550 & 21 & 14 & 4 \\
\hline $\begin{array}{l}30 \\
30\end{array}$ & 5 & $\begin{array}{l}60 \\
85\end{array}$ & $\begin{array}{l}277.1 \\
227.35\end{array}$ & $277.10^{270}$ & $\begin{array}{l}0.18 \\
0.24\end{array}$ & 1.90 & $\begin{array}{l}2.30 \\
2.82\end{array}$ & $\begin{array}{l}12.23 \\
14.78\end{array}$ & $\begin{array}{l}0.058 \\
0.055\end{array}$ & $\begin{array}{l}0.550 \\
0.517\end{array}$ & $\begin{array}{l}0.797 \\
0.963\end{array}$ & 19.7 & $\begin{array}{l}0.233 \\
0.272\end{array}$ & $\begin{array}{l}0.0166 \\
0.0208\end{array}$ & $\begin{array}{l}0.0635 \\
0.0365\end{array}$ & $\begin{array}{l}2.04 \\
2.42\end{array}$ & $\begin{array}{l}10 \\
12\end{array}$ & $\begin{array}{l}65 \\
87\end{array}$ & $\begin{array}{l}2224 \\
220\end{array}$ & $\begin{array}{l}74 \\
80\end{array}$ & $\begin{array}{l}46 \\
46\end{array}$ & $\begin{array}{l}1387 \\
1138\end{array}$ & $\begin{array}{r}27 \\
414\end{array}$ & $\begin{array}{l}23 \\
28\end{array}$ & $\begin{array}{l}14 \\
13\end{array}$ & 6 \\
\hline $\begin{array}{l}30 \\
30 \\
30\end{array}$ & $\begin{array}{l}5 \\
5\end{array}$ & $\begin{array}{r}85 \\
108\end{array}$ & $\begin{array}{l}2777.35 \\
277.58\end{array}$ & $\begin{array}{l}277.35^{\circ} \\
277.50^{\circ}\end{array}$ & 0.24 & $\begin{array}{l}2.21 \\
1.25\end{array}$ & $\begin{array}{l}2.82 \\
1.27\end{array}$ & $\begin{array}{r}14.78 \\
8.22\end{array}$ & $\begin{array}{l}0.0522 \\
0.061\end{array}$ & $\begin{array}{l}0.517 \\
0.317\end{array}$ & $\begin{array}{l}0.963 \\
0.411\end{array}$ & $\begin{array}{l}17.2 \\
27.4\end{array}$ & $\begin{array}{l}0.272 \\
0.114\end{array}$ & $\begin{array}{l}0.0208 \\
0.0133\end{array}$ & $\begin{array}{l}0.0635 \\
0.0636\end{array}$ & $\begin{array}{l}2.42 \\
1.01\end{array}$ & $\begin{array}{l}12 \\
59\end{array}$ & $\begin{array}{r}87 \\
180\end{array}$ & $\begin{array}{l}220 \\
290\end{array}$ & $\begin{array}{r}80 \\
179\end{array}$ & $\begin{array}{l}46 \\
18\end{array}$ & $\begin{array}{l}1138 \\
1678\end{array}$ & $\begin{array}{l}414 \\
257\end{array}$ & $\begin{array}{l}28 \\
23\end{array}$ & $\begin{array}{l}13 \\
14\end{array}$ & 0 \\
\hline
\end{tabular}


Table 3. Site 731 XRF data. " indicates unreliable trace element abundances.

\begin{tabular}{|c|c|c|c|c|c|c|c|c|c|c|c|c|c|c|c|c|c|c|c|c|c|c|c|c|c|c|c|c|}
\hline $\begin{array}{l}\text { Core } \\
\text { no. }\end{array}$ & $\begin{array}{l}\text { Sec } \\
\text { no. }\end{array}$ & $\begin{array}{c}\text { Sec } \\
\text { depth } \\
\text { (cm) }\end{array}$ & $\begin{array}{l}\text { ODP } \\
\text { depth } \\
\text { (mbsf) }\end{array}$ & $\begin{array}{l}\text { True } \\
\text { depth } \\
\text { (mbsf) }\end{array}$ & $\begin{array}{l}\mathrm{Na} \\
(\%)\end{array}$ & $\begin{array}{l}\mathrm{Mg}_{(\%)} \\
(\%)\end{array}$ & $\begin{array}{l}\mathrm{Al} \\
(\%)\end{array}$ & $\begin{array}{c}\mathrm{Si} \\
(\%)\end{array}$ & $\begin{array}{c}P \\
(\%)\end{array}$ & $\begin{array}{l}\mathrm{S} \\
(\%)\end{array}$ & $\underset{(\%)}{\mathrm{K}}$ & $\begin{array}{l}\mathrm{Ca} \\
(\%)\end{array}$ & $\begin{array}{c}\mathrm{Ti} \\
(\%)\end{array}$ & $\begin{array}{c}\mathrm{Cr} \\
(\%)\end{array}$ & $\begin{array}{l}\mathrm{Mn} \\
(\%)\end{array}$ & $\begin{array}{l}\mathrm{Fe} \\
(\%)\end{array}$ & $\begin{array}{c}\text { Co } \\
(\mathrm{ppm})\end{array}$ & $\begin{array}{c}\mathrm{Ni} \\
(\mathrm{ppm})\end{array}$ & $\begin{array}{c}\mathrm{Cu} \\
\text { (ppm) }\end{array}$ & $\underset{(\mathrm{ppm})}{\mathrm{Zn}}$ & $\begin{array}{c}\mathrm{Rb} \\
(\mathrm{ppm})\end{array}$ & $\begin{array}{c}\mathrm{Sr} \\
(\mathrm{ppm})\end{array}$ & $\begin{array}{c}\mathrm{Ba} \\
(\mathrm{ppm})\end{array}$ & $\underset{(\mathrm{ppm})}{\mathbf{L a}}$ & $\begin{array}{c}\mathrm{Pb} \\
(\mathrm{ppm})\end{array}$ & $\begin{array}{c}\mathrm{Th} \\
(\mathrm{ppm})\end{array}$ & $\underset{(\mathrm{ppm})}{\mathrm{U}}$ & $\begin{array}{l}\text { Salt } \\
(\%)\end{array}$ \\
\hline \multicolumn{29}{|c|}{ Hole $731 \mathrm{~A}$} \\
\hline 32 & 1 & 33 & 299.53 & $299.53^{\circ}$ & 0.25 & 1.31 & 2.11 & 7.6 & 0.007 & 0.017 & 0.883 & 28.3 & 0.143 & 0 & 0.0146 & 1.5 & 5 & 12 & 239 & 32 & 32 & 1439 & 417 & 27 & 14 & 0 & 3 & 0.69 \\
\hline 32 & 1 & 60 & 299.8 & $299.8^{\circ}$ : & 0.22 & 1.54 & 1.89 & ${ }^{7}$ & 0 & 0.021 & 0.741 & 29.2 & 0.126 & 0 & 0.0157 & 1.32 & 1. & 12 & 238 & 40 & 27 & 1225 & $\begin{array}{r}69 \\
69\end{array}$ & 28 & 14 & 0 & 3 & 0.59 \\
\hline 32 & 1 & 90 & 300.1 & $300.1^{\circ}$ & 0.29 & 2.47 & 3.07 & 12.2 & 0.003 & 0.032 & 1.216 & 21 & 0.227 & 0.0048 & 0.0149 & 2.36 & 12 & 25 & 227 & 54 & 44 & 1290 & 189 & 19 & 13 & 3 & 3 & 0.74 \\
\hline 32 & 1 & 120 & 300.4 & $300.4^{\circ}$ & 0.17 & 0.81 & 1.16 & 3.9 & 0 & 0.014 & 0.425 & 34.1 & 0.073 & & 0.0011 & 0.67 & 1 & 13 & 247 & 7 & 18 & 1393 & 31 & 22 & 14 & 0 & 3 & 0.61 \\
\hline 32 & 1 & 149 & 300.69 & $300.69^{\circ}$ & 0.2 & 0.93 & 1.65 & 5.5 & 0 & 0.014 & 0.637 & 31.8 & 0.101 & 0 & 0.0017 & 1.07 & 1 & 13 & 244 & 16 & 26 & 1352 & 291 & 32 & 14 & 0 & 3 & 0.81 \\
\hline 32 & 2 & 30 & 301 & 301 & 0.16 & 0.9 & 1.29 & 4.5 & 0 & 0.017 & 0.468 & 33.3 & 0.086 & 0 & 0.0074 & 0.83 & 3 & 13 & 246 & 10 & 20 & 1279 & 31 & 32 & 14 & 1 & 3 & 0.83 \\
\hline 32 & 2 & 60 & 301.3 & $301.3^{\circ}$ & 0.33 & 2.49 & 3.36 & 13.3 & 0 & 0.033 & 1.363 & 19.5 & 0.264 & 0.0062 & 0.0073 & 2.72 & 15 & 59 & 225 & 61 & 52 & 1263 & 51 & 35 & 13 & 2 & 3 & 0.69 \\
\hline 32 & 2 & 90 & 301.6 & $301.6^{\circ}$ & 0.24 & 1.12 & 1.95 & 6.9 & 0 & 0.018 & 0.779 & 29.5 & 0.139 & & 0.0038 & 1.43 & 6 & 12 & 240 & 34 & 31 & 1258 & 329 & 39 & 14 & 0 & 3 & 0.56 \\
\hline 32 & 2 & 120 & 301.9 & $301.9^{\circ}$ & 0.32 & 1.44 & 2.5 & 9.4 & 0.005 & 0.031 & 1.054 & 25.8 & 0.194 & 0.0007 & 0.0099 & 2.05 & 11 & 12 & 235 & 59 & 36 & 1250 & 41 & 30 & 14 & 0 & 3 & 0.52 \\
\hline 32 & 2 & 149 & 302.19 & $302.19^{\circ}$ & 0.21 & 1.94 & 2.41 & 9.3 & 0.001 & 0.03 & 0.91 & 25.8 & 0.175 & 0.0015 & 0.0157 & 1.88 & 8 & 12 & 235 & 79 & 31 & 11 & 368 & 27 & 14 & 0 & 3 & 0.72 \\
\hline 32 & 3 & 30 & 302.5 & $302.50^{\circ}$ & 0.17 & 1.15 & 1.83 & $\begin{array}{l}6.5 \\
5.5\end{array}$ & 0 & 0.016 & 0.736 & 29.9 & 0.121 & 0 & 0.063 & 1.29 & 8 & 12 & 241 & 41 & 26 & 1363 & 30 & 28 & 14 & 0 & 3 & 0.64 \\
\hline 32 & 3 & $\begin{array}{l}60 \\
88\end{array}$ & 302.8 & $302.8^{\circ} 0^{\circ}$ & 0.15 & 0.97 & 1.64 & 5.5 & ${ }_{0}^{0}$ & 0.014 & 0.664 & 31.7 & 0.11 & 0 & 0.0056 & 1.08 & 5 & 13 & 244 & 17 & 22 & 1179 & 30 & 23 & 14 & 0 & 3 & 0.78 \\
\hline 32 & 3 & $\begin{array}{r}88 \\
118\end{array}$ & $\begin{array}{l}303.08 \\
303.38\end{array}$ & $\begin{array}{l}303.08^{\circ} \\
303\end{array}$ & 0.18 & 0.98 & $\begin{array}{l}1.64 \\
3.52\end{array}$ & 5.6 & ${ }^{0}$ & 0.016 & 0.657 & 31.5 & 0.108 & & 0.0084 & 1.06 & 4 & 13 & 243 & 34 & 25 & 1186 & 30 & 24 & 14 & 0 & 3 & 0.62 \\
\hline 32 & 3 & $\begin{array}{l}118 \\
180\end{array}$ & $\begin{array}{l}303.38 \\
303.7\end{array}$ & $\begin{array}{l}303.38^{\circ} \\
303 .\end{array}$ & 0.34 & $\begin{array}{l}1.78 \\
1.33\end{array}$ & $\begin{array}{l}3.52 \\
2.34\end{array}$ & $\begin{array}{l}12.4 \\
8.2\end{array}$ & 0.012 & $\begin{array}{l}0.013 \\
0.012\end{array}$ & $\begin{array}{l}1.445 \\
0.995\end{array}$ & 212 & 0.24 & 0.0046 & $\begin{array}{l}0.0124 \\
0.0058\end{array}$ & 2.63 & 14 & 12 & 227 & 68 & 56 & 1100 & 27 & 22 & ${ }^{13}$ & 0 & 3 & 0.57 \\
\hline 32 & 3 & $\begin{array}{l}150 \\
33\end{array}$ & $\begin{array}{l}303.7 \\
30403\end{array}$ & $303.70^{\circ}$ & 0.24 & 1.33 & 2.34 & $\begin{array}{l}8.2 \\
70\end{array}$ & 0.001 & 0.012 & 0.995 & 27.2 & 0.165 & & 0.0058 & 1.73 & $\begin{array}{r}5 \\
62\end{array}$ & 12 & 23 & 27 & 36 & 1184 & 29 & 27 & 14 & 2 & 3 & 0.55 \\
\hline 32 & $\begin{array}{l}4 \\
4\end{array}$ & $\begin{array}{l}33 \\
60\end{array}$ & $\begin{array}{l}304.03 \\
304.3\end{array}$ & $\begin{array}{l}304.03^{*} \\
3\end{array}$ & & $\begin{array}{l}1.31 \\
2.45\end{array}$ & $\begin{array}{l}2.14 \\
3.16\end{array}$ & $\begin{array}{r}7.9 \\
12.3\end{array}$ & $\begin{array}{l}0.005 \\
0.005\end{array}$ & $\begin{array}{l}0.02 \\
0.024\end{array}$ & $\begin{array}{l}0.928 \\
1.315\end{array}$ & $\begin{array}{l}27.9 \\
20.9\end{array}$ & $\begin{array}{l}0.145 \\
0.229\end{array}$ & $\begin{array}{l}0.0047 \\
0.01\end{array}$ & $\begin{array}{l}0.0204 \\
0.0357\end{array}$ & $\begin{array}{l}1.68 \\
2.57\end{array}$ & 62 & 172 & $\begin{array}{l}311 \\
300\end{array}$ & 180 & $\begin{array}{l}18 \\
17\end{array}$ & $\begin{array}{l}1096 \\
978\end{array}$ & 29 & $\begin{array}{l}27 \\
36\end{array}$ & ${ }_{13}^{14}$ & 0 & 3 & 0.51 \\
\hline $\begin{array}{l}32 \\
32\end{array}$ & $\begin{array}{l}4 \\
4\end{array}$ & $\begin{array}{l}60 \\
90\end{array}$ & $\begin{array}{l}304.3 \\
304.6\end{array}$ & $\begin{array}{l}304.3^{\circ} \\
3040^{\circ}\end{array}$ & 0.01 & $\begin{array}{l}2.45 \\
1.31\end{array}$ & $\begin{array}{l}3.16 \\
2.19\end{array}$ & $\frac{12.3}{8}$ & $\begin{array}{l}0.005 \\
0.002\end{array}$ & $\begin{array}{l}0.024 \\
0.018\end{array}$ & $\begin{array}{l}1.315 \\
0.946\end{array}$ & $\begin{array}{l}20.9 \\
27.7\end{array}$ & $\begin{array}{l}0.229 \\
0.147\end{array}$ & $\begin{array}{l}0.01 \\
0.0042\end{array}$ & $\begin{array}{l}0.0357 \\
0.0155\end{array}$ & $\begin{array}{l}2.57 \\
1.67\end{array}$ & $\begin{array}{l}65 \\
63\end{array}$ & $\begin{array}{l}192 \\
172\end{array}$ & $\begin{array}{l}300 \\
311\end{array}$ & $\begin{array}{l}190 \\
176\end{array}$ & $\begin{array}{l}17 \\
18\end{array}$ & $\begin{array}{r}978 \\
1247\end{array}$ & $\begin{array}{l}277 \\
29\end{array}$ & $\begin{array}{l}36 \\
38\end{array}$ & ${ }_{14}^{13}$ & $\begin{array}{l}0 \\
0\end{array}$ & 3 & 0.66 \\
\hline 32 & $\begin{array}{l}4 \\
4\end{array}$ & $\begin{array}{l}90 \\
120\end{array}$ & $\begin{array}{l}304.6 \\
304.9\end{array}$ & $\begin{array}{l}304.6 \% \\
304.9\end{array}$ & & $\begin{array}{l}1.31 \\
0.86\end{array}$ & $\begin{array}{l}2.19 \\
1.27\end{array}$ & $\begin{array}{l}8 \\
4.6\end{array}$ & $\begin{array}{l}0.002 \\
0\end{array}$ & $\begin{array}{l}0.018 \\
0.016\end{array}$ & $\begin{array}{l}0.946 \\
0.553\end{array}$ & $\begin{array}{l}27.7 \\
32.9\end{array}$ & $\begin{array}{l}0.147 \\
0.087\end{array}$ & $\begin{array}{l}0.00022 \\
0.0016\end{array}$ & $\begin{array}{l}0.01155 \\
0.013\end{array}$ & $\begin{array}{l}1.67 \\
0.98\end{array}$ & $\begin{array}{l}63 \\
59\end{array}$ & $\begin{array}{l}172 \\
141\end{array}$ & $\begin{array}{l}311 \\
311\end{array}$ & $\begin{array}{l}176 \\
157\end{array}$ & $\begin{array}{l}18 \\
18\end{array}$ & $\begin{array}{l}1247 \\
1224\end{array}$ & $\begin{array}{l}29 \\
31\end{array}$ & $\begin{array}{l}38 \\
23\end{array}$ & $\begin{array}{l}14 \\
14\end{array}$ & $\begin{array}{l}0 \\
0\end{array}$ & 3 & \\
\hline 32 & 4 & 149 & 305.19 & $305.19^{\circ}$ & & 0.83 & 1.17 & 4.1 & 0 & 0.017 & 0.46 & 33.9 & 0.071 & 0.001 & 0.016 & 0.81 & $\begin{array}{l}59 \\
58\end{array}$ & $\begin{array}{l}141 \\
131\end{array}$ & $\begin{array}{l}311 \\
304\end{array}$ & 144 & $\begin{array}{l}18 \\
18\end{array}$ & 1203 & $\begin{array}{l}31 \\
31\end{array}$ & $\begin{array}{l}23 \\
22\end{array}$ & $\begin{array}{l}14 \\
14\end{array}$ & 0 & ${ }_{3}^{3}$ & $\begin{array}{l}0.48 \\
0.53\end{array}$ \\
\hline 32 & 5 & 30 & 305.5 & $305.5^{\circ}$ & & 1.72 & 2.16 & 8 & 0 & 0.02 & 0.875 & 27.4 & 0.142 & 0.0057 & 0.033 & 1.58 & 60 & 168 & 293 & 162 & 18 & 1130 & 81 & 23 & 14 & 0 & 3 & 0.47 \\
\hline 32 & 5 & 60 & 305.8 & $305.8^{-}$ & & 1.37 & 1.88 & 6.9 & 0 & 0.02 & 0.785 & 29.1 & 0.124 & 0.0042 & 0.0206 & 1.29 & 61 & 171 & 341 & 175 & 18 & 1345 & 30 & 22 & 14 & 0 & 3 & 0.47 \\
\hline 32 & 5 & 90 & 306.1 & $306.1^{-}$ & & 0.71 & 0.97 & 3.2 & 0 & 0.016 & 0.358 & 35 & 0.053 & 0.0008 & 0.0255 & 0.6 & 59 & 145 & 304 & 146 & 18 & 1305 & 31 & 23 & 14 & 0 & 3 & 0.87 \\
\hline 32 & 5 & 120 & 306.4 & $306.4^{\circ}$ & & 0.72 & 1.02 & 3.3 & 0 & 0.016 & 0.387 & 34.7 & 0.059 & 0.0013 & 0.0312 & 0.64 & 59 & 136 & 299 & 151 & 18 & 1291 & 31 & 21 & 14 & 0 & 3 & 0.82 \\
\hline 32 & 5 & 149 & 306.69 & $306.69^{\circ}$ & & 0.57 & 0.74 & 2.3 & 0 & 0.013 & 0.259 & 36.7 & 0.042 & 0 & 0.0235 & 0.46 & 57 & 118 & 290 & 135 & 18 & 1306 & 32 & 23 & 14 & 0 & 3 & 0.61 \\
\hline 32 & 6 & 30 & 307 & 307 - & & 0.55 & 0.68 & 2.1 & 0 & 0.014 & 0.211 & 37.1 & 0.036 & 0 & 0.0249 & 0.41 & 58 & 138 & 291 & 135 & 18 & 1283 & 32 & 22 & 14 & 0 & 3 & 0.75 \\
\hline 32 & 6 & 60 & 307.3 & $307.3^{\circ}$ & & 0.62 & 0.81 & 2.6 & 0 & 0.016 & 0.268 & 36.1 & 0.044 & 0.0011 & 0.0281 & 0.5 & 59 & 131 & 323 & 145 & 18 & 1281 & 32 & 29 & 14 & 0 & 3 & 0.65 \\
\hline 32 & 6 & 90 & 307.6 & $307.6^{\circ}$ & 0.39 & 2.75 & 5.76 & 20.9 & 0.023 & 0.015 & 2.261 & 9.4 & 0.406 & 0.0175 & 0.039 & 4.61 & 73 & 270 & 290 & 264 & 16 & 504 & 177 & 31 & 12 & 0 & 3 & 0.53 \\
\hline 32 & 6 & 120 & 307.9 & $307.9^{\circ}$ & & 0.69 & 0.91 & 3 & 0 & 0.015 & 0.331 & 35.5 & 0.049 & 0.0005 & 0.0291 & 0.56 & 57 & 131 & 284 & 141 & 18 & 1295 & 31 & 41 & 14 & 0 & 3 & 0.55 \\
\hline 32 & CC & 30 & 308.5 & $308.5^{\circ}$ & 0.16 & 0.7 & 1.06 & 3.4 & 0 & 0.013 & 0.366 & 35.1 & 0.069 & & 0.015 & 0.66 & 0 & 13 & 249 & 3 & 18 & 1324 & 59 & 33 & 14 & 0 & 3 & 0.61 \\
\hline 37 & 1 & 30 & 341.6 & $341.6^{\circ}$ & 0.49 & 3.25 & 7.43 & 24.4 & 0.024 & 0.014 & 2.815 & 4.3 & 0.52 & 0.0238 & 0.1697 & 6.11 & 79 & 329 & 316 & 290 & 15 & 257 & 22 & 18 & 12 & 0 & 3 & 0.57 \\
\hline 37 & 1 & 60 & 341.9 & $341.9^{\circ}$ & 0.53 & 3.38 & 7.72 & 25 & 0.025 & 0.014 & 2.941 & 2.7 & 0.535 & 0.0254 & 0.2723 & 7.02 & 84 & 343 & 34 & 291 & 15 & 124 & 22 & 17 & 12 & 0 & 2 & 0.59 \\
\hline 37 & 1 & 90 & 342.2 & $342.2^{\circ}$ & 0.35 & 2.64 & 5.35 & 18.2 & 0.029 & 0.022 & 2.042 & 12 & 0.389 & 0.0162 & 0.2577 & 5.09 & 74 & 23 & 3 & 233 & 16 & 9 & 123 & 23 & 13 & 0 & 3 & 0.68 \\
\hline 37 & 1 & 120 & 342.5 & $342.5^{\circ}$ & 0.15 & 2.56 & 4.61 & 16.2 & 0.012 & 0.021 & 1.78 & 14.8 & 0.321 & 0.0128 & 0.4328 & 3.6 & 51 & 2. & 2 & 22 & 16 & 10 & 71 & 27 & 13 & 0 & 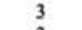 & 0.63 \\
\hline 37 & 1 & 150 & 342.8 & $342.8^{\circ}$ & 0.43 & 3.11 & 6.93 & 22.6 & 0.0 & 0.014 & 2.7 & 6.1 & 0.465 & 0.02 & 0.1 & 6.05 & 66 & 3 & 3 & 27 & 16 & 32 & 242 & 21 & 12 & 0 & 3 & 0.56 \\
\hline 37 & 2 & 30 & 343.1 & $343.1^{\circ}$ & 0.44 & 3.4 & 7.75 & 23.6 & 0.013 & 0.013 & 3.084 & 3.9 & 0.492 & 0.0256 & 0.1672 & 6. & 70 & 3 & $3 c$ & 2 & 15 & 1 & 22 & 15 & 12 & 0 & 3 & 0.58 \\
\hline 37 & 2 & 60 & 343.4 & $343.4^{\circ}$ & 0.48 & 3.5 & 6.71 & 22. & & 0.017 & & 6.6 & 0.464 & & & & 66 & , & & 3 & 16 & & 23 & 15 & 12 & 0 & 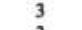 & 0.59 \\
\hline 37 & 2 & 90 & 343.7 & $343.7^{\circ}$ & 0.41 & 2.82 & 6.34 & 20.7 & $0 . c$ & 0.019 & 2. & 8.5 & 0.432 & 0.0 & 0.2 & & 63 & 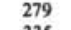 & 3 & 2 & 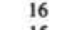 & 53 & 97 & 16 & 2 & 0 & 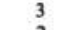 & 0.07 \\
\hline 37 & 2 & 120 & 344 & $344:$ & 0.65 & 3.2 & 7.57 & 25.7 & 0. & 0.013 & 2.78 & 3 & 0.552 & 0.0263 & 0.2 & & 84 & 33 & 31 & 2 & 5 & 111 & 41 & 16 & 12 & 0 & 2 & 0.51 \\
\hline 37 & 2 & 150 & 344.3 & $344.3^{\circ}$ & 0.53 & 3.22 & 7.41 & 24.1 & 0.027 & 0.014 & 2.794 & 4 & 0.508 & 0.0228 & 0.2198 & 6. & 82 & 3 & 3 & 28 & 15 & 252 & 22 & 21 & 12 & 0 & 3 & .57 \\
\hline 37 & 3 & 30 & 344.6 & $344.6^{\circ}:$ & 0.61 & 3.53 & 8.08 & 25.7 & 0.029 & 0.013 & $\begin{array}{l}3.039 \\
2.605\end{array}$ & 1.7 & 0.55 & 0.0261 & 0.2 & 7. & 87 & 33 & 3 & 2 & 15 & & 50 & 20 & 12 & 0 & 2 & 0.65 \\
\hline 37 & 3 & 60 & 344.9 & $344.9^{\circ}:$ & 0.6 & 3.17 & $\begin{array}{l}7.14 \\
678\end{array}$ & 24.9 & 0.032 & 0.015 & 2.605 & 4.1 & 0.526 & 0.0229 & 0.1 & 5. & 80 & 29 & 3 & 31 & 15 & 224 & 22 & 29 & 12 & 0 & 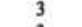 & 56 \\
\hline 37 & 3 & 90 & 345.2 & $345.2^{\circ}$ & 0.64 & 3.01 & $\begin{array}{l}6.78 \\
5.55\end{array}$ & 26 & $\begin{array}{l}0.041 \\
0.019\end{array}$ & $\begin{array}{l}0.012 \\
0.024\end{array}$ & 2.384 & 4 & $\begin{array}{l}0.534 \\
0.03\end{array}$ & $\begin{array}{l}0.0237 \\
0.0163\end{array}$ & 0.1 & 5. & 75 & 2 & 27 & 2 & 15 & 10 & 114 & 29 & 12 & 0 & 3 & 37 \\
\hline 37 & 3 & 120 & $\begin{array}{l}345.5 \\
3457\end{array}$ & $345.5^{\circ}$ & $\begin{array}{l}0.5 \\
0.93\end{array}$ & $\begin{array}{l}3.14 \\
3.18\end{array}$ & 5.55 & $\begin{array}{l}19.4 \\
25.3\end{array}$ & 0.019 & 0.024 & $\begin{array}{l}1.973 \\
2.278\end{array}$ & 11.2 & $\begin{array}{l}0.403 \\
0.59\end{array}$ & 0.0163 & 0.3 & 4. & 74 & 2 & $\begin{array}{l}308 \\
3,32\end{array}$ & 2 & 16 & 951 & $\begin{array}{l}593 \\
257\end{array}$ & 14 & ${ }_{12}^{12}$ & 0 & 3 & 91 \\
\hline $\begin{array}{l}37 \\
37\end{array}$ & 3 & $\begin{array}{r}149 \\
30\end{array}$ & $\begin{array}{l}345.79 \\
346.1\end{array}$ & $\begin{array}{l}345.79^{*} \\
346,\end{array}$ & $\begin{array}{l}0.93 \\
0.46\end{array}$ & $\begin{array}{l}3.18 \\
2.63\end{array}$ & $\begin{array}{l}6.23 \\
6.12\end{array}$ & $\begin{array}{l}25.3 \\
20.1\end{array}$ & $\begin{array}{l}0.06 \\
0.033\end{array}$ & $\begin{array}{l}0.015 \\
0.019\end{array}$ & $\begin{array}{l}2.278 \\
2.291\end{array}$ & $\begin{array}{l}5.4 \\
9.8\end{array}$ & $\begin{array}{l}0.559 \\
0.426\end{array}$ & $\begin{array}{l}0.0239 \\
0.0173\end{array}$ & $\begin{array}{l}0.1588 \\
0.2861\end{array}$ & 4. & ${ }^{73}$ & 2 & 3. & 27 & 15 & 194 & 257 & ${ }_{25}^{14}$ & ${ }_{12}^{12}$ & 0 & 3 & 54 \\
\hline $\begin{array}{l}37 \\
37\end{array}$ & $\begin{array}{l}4 \\
4\end{array}$ & $\begin{array}{l}30 \\
60\end{array}$ & $\begin{array}{l}3466.1 \\
346.4\end{array}$ & $\begin{array}{l}346.1 \\
346.4\end{array}$ & $\begin{array}{l}0.46 \\
0.45\end{array}$ & $\begin{array}{l}2.63 \\
3.38\end{array}$ & $\begin{array}{l}6.12 \\
7.73\end{array}$ & $\begin{array}{l}20.1 \\
24.6\end{array}$ & $\begin{array}{l}0.0 .023 \\
0.025\end{array}$ & $\begin{array}{l}0.019 \\
0.013\end{array}$ & $\begin{array}{l}2.291 \\
2.963\end{array}$ & $\begin{array}{l}9.8 \\
3.7\end{array}$ & $\begin{array}{l}0.426 \\
0.517\end{array}$ & $\begin{array}{l}0.01733 \\
0.0243\end{array}$ & $\begin{array}{l}0.2861 \\
0.1869\end{array}$ & $\begin{array}{l}5.35 \\
6.56\end{array}$ & $\begin{array}{l}77 \\
82\end{array}$ & $\begin{array}{l}2644 \\
343\end{array}$ & ${ }_{36}^{37}$ & $\begin{array}{l}258 \\
293\end{array}$ & $\begin{array}{l}16 \\
15\end{array}$ & $\begin{array}{l}611 \\
111\end{array}$ & $\begin{array}{r}434 \\
22\end{array}$ & $\begin{array}{l}25 \\
19\end{array}$ & $\begin{array}{l}12 \\
12\end{array}$ & $\begin{array}{l}0 \\
0\end{array}$ & $\begin{array}{l}3 \\
2\end{array}$ & $\begin{array}{l}.74 \\
.55\end{array}$ \\
\hline 37 & 4 & 88 & 346.68 & $346.68^{\circ}$ & 0.55 & 3.1 & 7.22 & 23.4 & 0.034 & 0.015 & 2.634 & 5.1 & 0.511 & 0.0226 & 0.3345 & 6.5 & 83 & 305 & 34 & 282 & is & 406 & 401 & 15 & 12 & 0 & 3 & 0.68 \\
\hline 37 & 5 & 30 & 347.6 & $347.27^{\circ}$ & 0.57 & 3.4 & 7.58 & 25.5 & 0.027 & 0.013 & 2.835 & 3.1 & 0.527 & 0.0249 & 0.2551 & 6.29 & 81 & 324 & 384 & 294 & 15 & 87 & 22 & 14 & 12 & 0 & 2 & 0.56 \\
\hline 37 & 5 & 59 & 347.89 & $347.56^{\circ}$ & 0.38 & 3.12 & 5.77 & 20.4 & 0.027 & 0.023 & 2.161 & 9.1 & 0.414 & 0.0185 & 0.3964 & 5.15 & 77 & 271 & 366 & 252 & 16 & 570 & 576 & 19 & 13 & 0 & 3 & 0.68 \\
\hline 37 & 5 & 90 & 348.2 & $347,87^{*}$ & 0.51 & 3.3 & 7.55 & 24.8 & 0.024 & 0.013 & 2.904 & 3.5 & 0.521 & 0.0243 & 0.131 & 6.54 & 83 & 326 & 305 & 294 & 15 & 101 & 22 & 29 & 12 & 0 & 3 & 0.49 \\
\hline 37 & 5 & 120 & 348.5 & $348.17^{*}$ & 0.53 & 3.09 & 7.12 & 23.4 & 0.014 & 0.015 & 2.745 & 5.6 & 0.485 & 0.0227 & 0.2227 & 5.81 & 82 & 328 & 319 & 288 & 15 & 331 & 23 & 30 & 12 & 0 & 3 & 0.54 \\
\hline
\end{tabular}



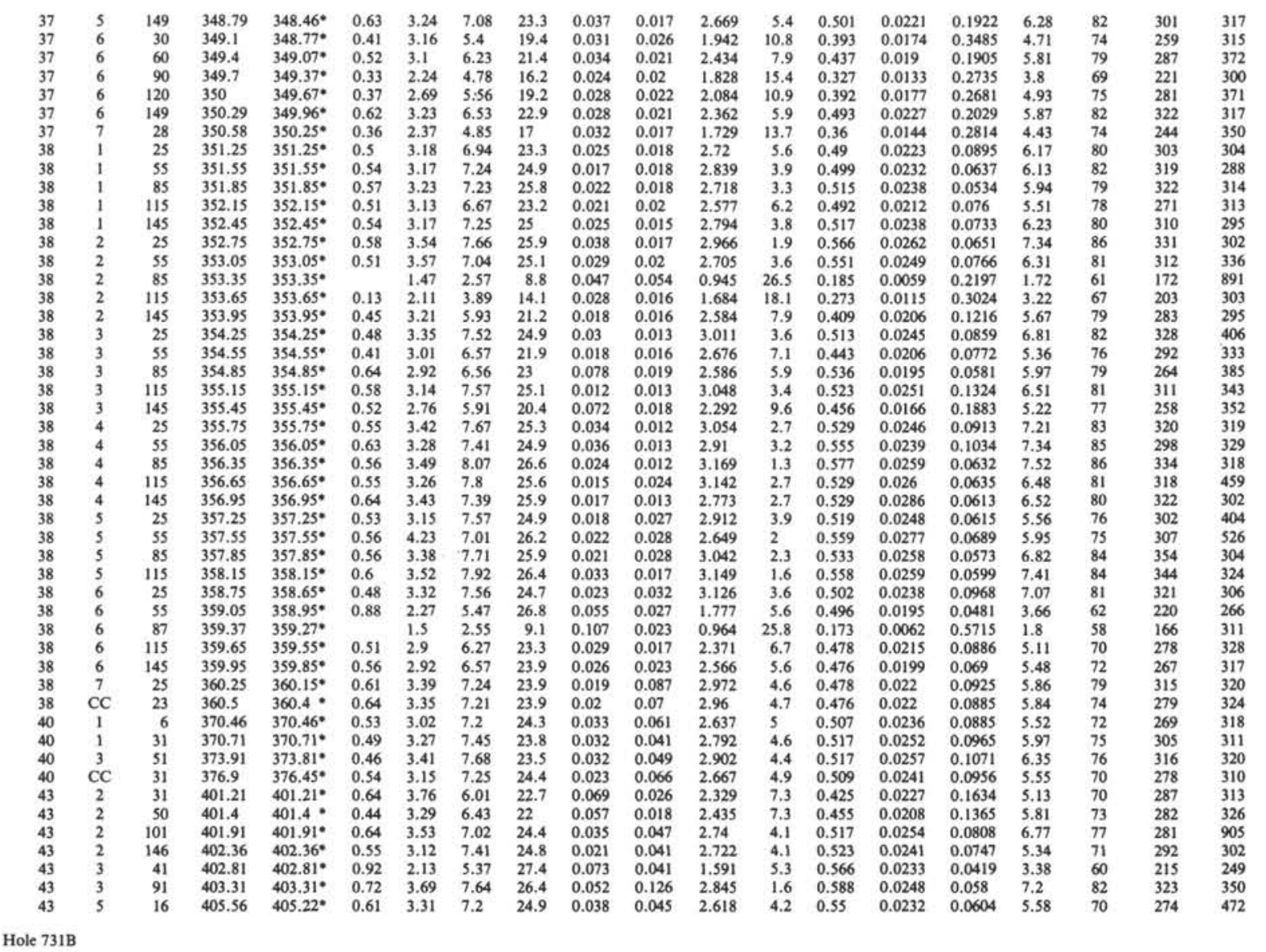

$\begin{array}{rrrrrrlll}287 & 15 & 393 & 331 & 24 & 12 & 0 & 3 & 0.78 \\ 246 & 16 & 827 & 509 & 22 & 12 & 0 & 3 & 0.71 \\ 266 & 16 & 496 & 552 & 25 & 12 & 0 & 3 & 0.64 \\ 223 & 16 & 1213 & 26 & 34 & 13 & 0 & 3 & 0.61 \\ 252 & 16 & 817 & 600 & 32 & 13 & 0 & 3 & 0.59 \\ 287 & 16 & 489 & 569 & 30 & 12 & 0 & 3 & 0.75 \\ 244 & 16 & 1057 & 25 & 23 & 13 & 0 & 3 & 0.64 \\ 271 & 15 & 442 & 157 & 16 & 12 & 0 & 3 & 0.69 \\ 272 & 15 & 156 & 22 & 14 & 12 & 0 & 3 & 0.51 \\ 311 & 15 & 151 & 71 & 14 & 12 & 0 & 2 & 0.62 \\ 276 & 15 & 543 & 284 & 16 & 12 & 0 & 3 & 0.72 \\ 291 & 15 & 283 & 22 & 19 & 12 & 0 & 3 & 0.62 \\ 306 & 15 & 90 & 22 & 23 & 12 & 0 & 2 & 0.77 \\ 284 & 15 & 264 & 22 & 20 & 12 & 0 & 3 & 0.81 \\ 185 & 17 & 1169 & 29 & 27 & 14 & 0 & 3 & 0.76 \\ 212 & 17 & 1249 & 27 & 28 & 13 & 0 & 3 & 0.62 \\ 264 & 16 & 404 & 23 & 31 & 12 & 0 & 3 & 0.68 \\ 274 & 15 & 105 & 22 & 19 & 12 & 0 & 2 & 0.47 \\ 282 & 16 & 384 & 23 & 20 & 12 & 0 & 3 & 0.55 \\ 281 & 16 & 525 & 128 & 30 & 12 & 0 & 3 & 0.82 \\ 279 & 15 & 135 & 410 & 15 & 12 & 0 & 2 & 0.49 \\ 266 & 16 & 692 & 24 & 15 & 12 & 0 & 3 & 0.73 \\ 292 & 15 & 109 & 22 & 14 & 12 & 0 & 2 & 0.53 \\ 293 & 15 & 204 & 22 & 17 & 12 & 0 & 3 & 0.63 \\ 299 & 15 & 26 & 21 & 18 & 12 & 0 & 2 & 0.5 \\ 290 & 15 & 67 & 80 & 29 & 12 & 0 & 2 & 0.46 \\ 2281 & 15 & 87 & 22 & 17 & 12 & 0 & 2 & 0.5 \\ 289 & 15 & 88 & 62 & 15 & 12 & 0 & 3 & 0.53 \\ 282 & 15 & 102 & 22 & 18 & 12 & 0 & 2 & 0.89 \\ 293 & 15 & 50 & 22 & 15 & 12 & 0 & 2 & 0.58 \\ 299 & 15 & 43 & 21 & 14 & 12 & 0 & 2 & 0.71 \\ 274 & 15 & 124 & 22 & 15 & 12 & 0 & 2 & 0.59 \\ 2211 & 15 & 128 & 169 & 15 & 12 & 0 & 3 & 0.45 \\ 156 & 17 & 1374 & 29 & 24 & 14 & 0 & 3 & 0.7 \\ 266 & 15 & 417 & 84 & 24 & 12 & 0 & 3 & 0.53 \\ 269 & 15 & 365 & 118 & 16 & 12 & 0 & 3 & 0.52 \\ 273 & 15 & 155 & 23 & 14 & 12 & 0 & 3 & 0.5 \\ 270 & 15 & 158 & 22 & 17 & 12 & 0 & 3 & 0.47 \\ 260 & 15 & 104 & 339 & 29 & 12 & 0 & 3 & 0.44 \\ 267 & 15 & 94 & 430 & 21 & 12 & 0 & 3 & 0.48 \\ 272 & 15 & 95 & 286 & 21 & 12 & 0 & 3 & 0.5 \\ 254 & 15 & 109 & 126 & 18 & 12 & 0 & 3 & 0.47 \\ 270 & 15 & 235 & 33 & 15 & 12 & 0 & 3 & 0.52 \\ 266 & 16 & 567 & 140 & 16 & 12 & 0 & 3 & 0.57 \\ 276 & 15 & 229 & 22 & 23 & 12 & 0 & 3 & 0.54 \\ 276 & 15 & 96 & 22 & 21 & 12 & 0 & 3 & 0.5 \\ 217 & 15 & 121 & 53 & 18 & 12 & 0 & 3 & 0.46 \\ 298 & 15 & 70 & 21 & 17 & 12 & 0 & 2 & 0.64 \\ 285 & 15 & 210 & 22 & 21 & 12 & 0 & 3 & 0.59\end{array}$

$\begin{array}{llllllllllllllllll}3 & 1 & 29 & 428.39 & 428.39^{*} & 0.73 & 3.6 & 4.72 & 23.5 & 0.086 & 0.295 & 1.316 & 7.6 & 0.478 & 0.0572 & 0.1786 & 3.99 & 61 \\ 3 & 1 & 60 & 428.7 & 428.7 & 0.48 & 3.28 & 7.06 & 23.7 & 0.083 & 0.039 & 2.513 & 5.7 & 0.497 & 0.0245 & 0.0803 & 5 & 68 \\ 3 & C C & 10 & 430.13 & 429.77^{*} & 0.78 & 2.87 & 5.61 & 25.5 & 0.035 & 0.09 & 1.743 & 5.3 & 0.445 & 0.0264 & 0.0406 & 3.75 & 60 \\ 5 & 1 & 51 & 447.91 & 447.91^{*} & 0.55 & 3.24 & 7.48 & 24.3 & 0.029 & 0.026 & 2.698 & 4.5 & 0.058 & 0.0242 & 0.0794 & 5.77 & 71 \\ 5 & 1 & 71 & 448.11 & 448.11^{*} & 0.53 & 3.11 & 7.34 & 24.1 & 0.034 & 0.03 & 2.655 & 4.9 & 0.49 & 0.0229 & 0.0904 & 5.65 & 70\end{array}$ Hole $731 \mathrm{C}$

$\begin{array}{lllllllllllllllll}12 & 1 & 89 & 792.39 & 792.39^{*} & 0.87 & 2.31 & 5.28 & 25.6 & 0.034 & 0.026 & 1.578 & 5 & 0.408 & 0.0204 & 0.0359 & 4.23\end{array}$

\begin{tabular}{lll}
169 & 254 \\
\hline 0 & 255 & 303
\end{tabular} 


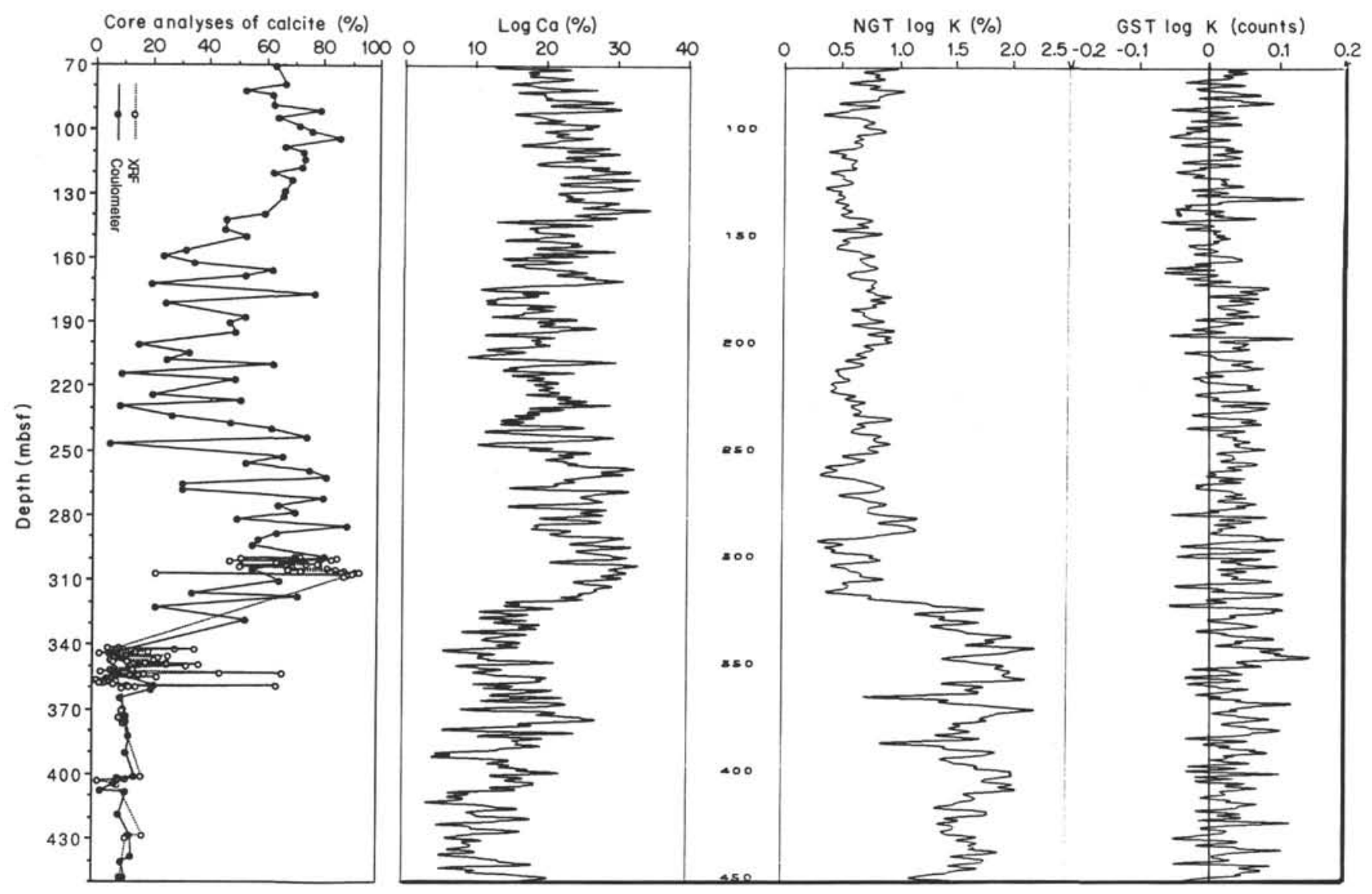

Figure 1. Comparisons among geochemical indicators at ODP Site 731, for 70-450 mbsf. Discrete core measurements of calcite percentage (Prell, Niitsuma, et al., 1989; this paper) confirm the overall accuracy of the calcium log; detailed comparison is not possible because of very different sampling volumes. The change from carbonates above 320 mbsf to terrigenous turbidites below 320 mbsf is evident on both the calcium and NGT potassium log. The GST potassium log (far right) appears to be unreliable.

The repartitioning of $\mathrm{Cl}$ and $\mathrm{K}$ has a significant but not overwhelming effect on the character of the other GST elements Ca, $\mathrm{Si}, \mathrm{Fe}, \mathrm{Ti}$, and $\mathrm{S}$ (e.g., Fig. 2). This repartitioning is expected to slightly improve the reliability of the geochemical logs.

Following repartitioning, the yields of $\mathrm{Ca}, \mathrm{Si}, \mathrm{Fe}, \mathrm{Ti}, \mathrm{S}$, and Gd were divided by (unfortunately proprietary) Schlumberger sensitivity coefficients, which convert from spectral yields to approximate elemental concentrations. For example, Gd yields are similar to those for $\mathrm{Ti}$, because of the huge $\mathrm{Gd}$ capture cross section; the sensitivity coefficients reduce the apparent Gd concentrations to $\mathrm{ppm}$ and $\mathrm{Ti}$ concentrations to $0 \%-1 \%$. Occasional negative concentrations are truncated at zero; except for $\mathrm{Ti}$ and $\mathrm{S}$ yields, this truncation was rarely needed. Finally, elemental concentrations were normalized by dividing by the sum of the oxide concentration. For this final step, $\mathrm{K}$ and $\mathrm{Al}$ oxides were included when available. For Sites 728 and 731 at which $\mathrm{Al}$ was not logged, an $\mathrm{Al} / \mathrm{Ti}$ ratio of 11.6 was used in this step; its effect on the character of resulting element logs is minor.

The log processing steps described above differ from the normal geochemical processing, in order to maximize the reliability obtainable independently of any XRF information. This difference decreases the direct applicability of our subsequent log/ XRF comparison to other ODP holes at which "normal" processing was utilized. However, a processing procedure similar to ours could be used on the other holes as well, if a similar type of analysis shows that it is appropriate.

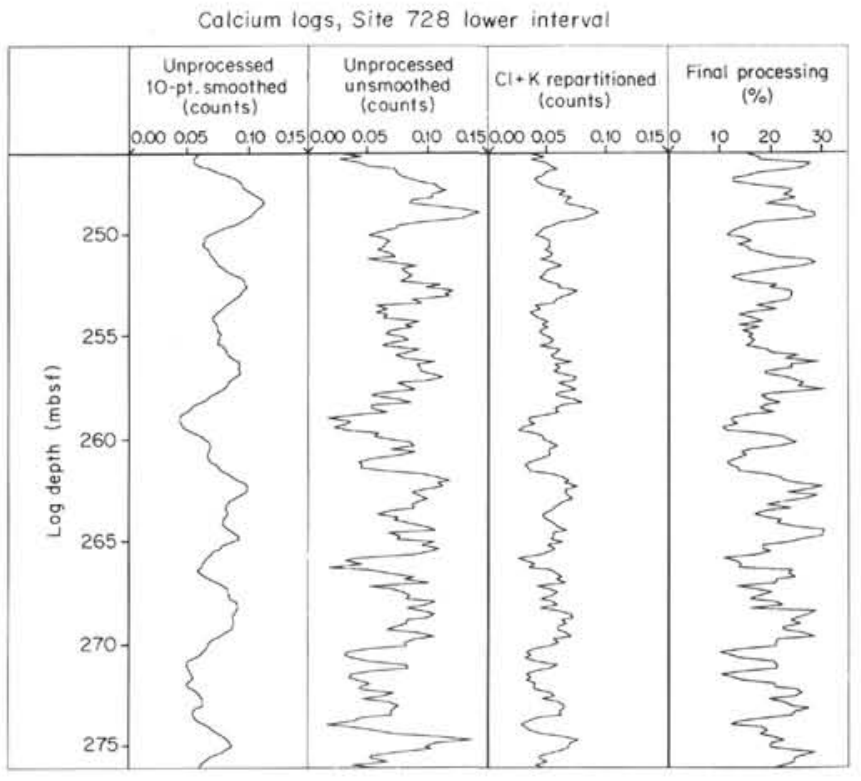

Figure 2. Effect of processing technique on the character of the calcium log. The leftmost curve is the normal processing employed for GST data; the rightmost curve is the final processing used here for comparison to XRF data. 


\section{COMPARISON OF XRF TO LOGS AS A FUNCTION OF DEPTH}

High-resolution sampling for XRF was undertaken for seven depth intervals, varying from 5 to $30 \mathrm{~m}$ in length. Three intervals were sampled at Site 723; two intervals were sampled at both Site 728 and Site 731. The number of XRF samples per interval (Table 4) varies dramatically, from a minimum of 18 samples in interval 4 to 123 samples in interval 5. Figures 3-9 show overlays of $\log$ and XRF measurements as a function of depth for each of the seven intervals, for each of the elements $\mathrm{Ca}, \mathrm{Si}$, $\mathrm{K}, \mathrm{Ti}, \mathrm{Fe}, \mathrm{S}$, and $\mathrm{Al}$.

The focus of these comparisons is on the character of geochemical variations as a function of depth, not on the absolute magnitudes of log-based elemental abundance in comparison to XRF-based abundance. Except perhaps for $\mathrm{K}$ and $\mathrm{Al}$ (which are reputed to be directly determined in volume percent), we do not expect our processing procedure to have accurately estimated concentrations. For example, by repartitioning all $\mathrm{K}$ counts rather than using the NGT to predict an average K count and repartitioning the residual from this average, we have effectively imparted a baseline rise in estimated abundances of $\mathrm{Ca}, \mathrm{Si}, \mathrm{S}$, and $\mathrm{Gd}$ and a drop in estimated abundances of $\mathrm{Ti}$ and $\mathrm{Fe}$. In the last portion of this section, we briefly examine the consistency of $\log$ and XRF magnitudes.

Depth uncertainty is the major limitation to almost any comparison of log and core data. Ship heave causes some uncertainty in both core and log depths; fortunately, seas were extremely calm during coring and logging of Sites 723,728 , and 731. Drill pipe stretch and particularly logging-cable stretch cause further mismatch; this mismatch is reduced by adjusting all initial log depths to a pipe standard, based on the log response to the base of pipe. Further correction for cable stretch can be accomplished by depth-shifting an entire log, based on matching similar features in logs and cores. For the comparisons of log and XRF abundance variations shown in Figures 39 and considered subsequently, we have depth-shifted all logs with respect to the core XRF data by a constant amount within each interval (Table 4). This slight depth shift (average $1.6 \mathrm{~m}$, maximum $3.8 \mathrm{~m}$ ) is based on matching of apparently correlative portions of XRF and $\log$ Ca peaks. Shift estimates are more similar for different intervals within a site than between sites, as expected because differential cable stretch is much less over short intervals than between holes with different water depths.

Incomplete core recovery is probably the biggest source of depth mismatch. Even with XRF sampling confined to highest recovery intervals, depth shifts of a meter or sometimes more are expected. Susceptibility correlations between overlapping piston cores show that " $100 \%$ " recovery cores are actually missing the top 10-30 cm of material (P. deMenocal, pers. comm.); apparently the piston coring process causes some core stretching. No comparable analysis of XCB cores has been undertaken. The ODP recording convention is to "hang" cores from the top of their cored interval, thereby assuming that any miss-

Table 4. Intervals with high-density XRF sampling.

\begin{tabular}{ccrrl}
\hline $\begin{array}{c}\text { Interval } \\
\text { no. }\end{array}$ & Hole & \multicolumn{1}{c}{$\begin{array}{c}\text { Core depths } \\
\text { (mbsf) }\end{array}$} & $\begin{array}{c}\text { Number } \\
\text { of XRD } \\
\text { samples }\end{array}$ & \multicolumn{1}{c}{ Lithology } \\
\hline 1 & $723 \mathrm{~B}$ & $92.74-102.85$ & 30 & Clayey carbonate \\
2 & $723 \mathrm{~B}$ & $208.65-233.68$ & 93 & Clayey carbonate \\
3 & $723 \mathrm{~B}$ & $381.25-386.10$ & 18 & Clayey carbonate \\
4 & $728 \mathrm{~B}$ & $178.55-182.80$ & 18 & Clayey carbonate \\
5 & $728 \mathrm{~B}$ & $247.75-277.35$ & 122 & Clayey carbonate \\
6 & $731 \mathrm{~A}$ & $299.53-308.50$ & 30 & Carbonate ooze \\
7 & $731 \mathrm{~A}$ & $341.60-360.15$ & 59 & Silty clay \\
\hline
\end{tabular}

ing material was lost from the bottom of the cored interval. As previously discussed, this procedure seems particularly appropriate for Site 723. Yet a comparison of XRF and log Ca concentrations for the middle interval from Site 723 (Fig. 10) shows that this assumption is only a good first approximation. Because of the high-resolution character of the XRF and log measurements, one can see that the top core in this interval needs to be shifted upward, indicating that the missing material comes from the top of the core rather than from the bottom. This interval is the only one in which a differential shift of XRF data was applied within an interval. Similar but perhaps smaller differential shifts are certainly present within the other six intervals; none were applied because the evidence was less compelling. Nevertheless, when visually comparing the character of $\mathrm{XRF}$ and $\log$ elemental-abundance variations on any of the plots of Figures 3-9, one must keep in mind that slight depth mismatches between peaks may or may not be caused by core recovery problems.

Our comparison is less hampered by the depth-shift problem than are some studies which relied on widely-spaced XRF measurements, because the high-resolution XRF character allows some refinement of depths. However, the best known comparison-the Conoco test well (Chapman et al., 1987) - was even less affected by depth shifts, because the stratigraphy there is characterized by several thick and relatively uniform-mineralogy beds, rather than our rapidly varying lithologies.

One further complication to a comparison of XRF and log geochemistry is the much different measurement volumes of the two methods. Our XRF measurement volumes were $10 \mathrm{~cm}^{3}$. In contrast, the geochemical tools average a volume of about $1 \mathrm{~m}^{3}$ and have a vertical averaging distance of about $0.5 \mathrm{~m}$. ODP GST logs routinely have a 10 -point $(1.52 \mathrm{~m})$ averaging applied for suppression of random noise, and the NGT logs use a variable-length (but generally shorter) Kalman filter. Intervals such as 4 and 5 exhibit a strong cyclicity with a wavelength of only about $2 \mathrm{~m}$, based on visual core descriptions, susceptibility core logging (Prell, Niitsuma, et al., 1989), and our XRF data. A 1.5 $\mathrm{m}$ smoothing is clearly too long for such intervals and seriously obscures detection of the major geochemical variability. Consequently, we use only a 3-point $(0.45 \mathrm{~m}) \log$ smoothing in Figures 3-9 and no smoothing at all in subsequent principal components analysis. The very mild log smoothing, high-resolution $(0.25-0.30 \mathrm{~m}) \mathrm{XRF}$ sampling, and gradual $(\mathrm{m})$ lithologic changes in the cores result in minimal problems of incompatible vertical resolutions between cores and logs. Examination of Figures 3-9 shows that almost all XRF peaks and troughs are defined by several adjacent XRF measurements rather than by a single measurement, so that these peaks and troughs are at least theoretically resolvable by the logs.

In the remainder of this section, we consider the comparison of XRF data with geochemical logs on an element-by-element basis. The largest lithologic change within the three sites is the downhole change from nannofossil ooze to terrigenous turbidites at Site 731. We begin discussion of each element with an examination of whether the geochemical logs reliably detect this major change. We then discuss the extent to which the logs have reproduced the small-scale variability within each of the seven intervals of Figures 3-9 and Table 4.

The primary criterion for this comparison is qualitative character match of this small-scale variability. In general, we neither expect nor obtain similar means or similar ranges of concentrations between XRF and geochemical logs, because proper scaling of the logs to elemental percentages is not possible unless all major elements are determined. However, Figures 3-8 are not scaled to facilitate character match; instead they have identical vertical scales for log and core data to optimize comparisons of means and standard deviations. A secondary and more quanti- 
tative comparison within each interval is the correlation coefficient. For calculation of the correlation coefficient, the reprocessed but unsmoothed element logs were resampled at the same depths as the XRF measurements. We omitted the first four XRF measurements from interval 1 and the first three XRF measurements from interval 3. XRF sampling for interval 1 was originally thought to have begun exactly at the start of openhole geochemical logging; however, the subseqently determined 1.2 $m$ depth shift between the two data sets means that no openhole logs are available at the top of interval 1. The element logs at the very top of interval 3 exhibit excursions to bizarre concentrations, caused by negative silicon yields which exaggerate excursions of all elements during the oxide normalization step. These obviously unreliable data are omitted in the statistical evaluation of the remainder of interval 3.

\section{Calcium}

Calcium is the only element for which a substantial corebased dataset exists in addition to our XRF data. Shipboard measurements of calcium carbonate percentage were made by coulometer at approximately 5-10 m intervals throughout Sites 723 and 728 and in the upper half of Site 731. The Site 723 and 728 carbonate data are of little value for our purposes, because both logs and XRF indicate that the vast majority of the carbonate variance resides in wavelengths of less than $5 \mathrm{~m}$. However, broad variations in carbonate are seen in the coulometer results from Site 731 .

Figure 1 plots both the 87 coulometer measurements and 106 XRF measurements of carbonate for the interval $70-450$ mbsf; 70 mbsf is just below the base of pipe for geochemical logging, and 450 mbsf is the change from continuous coring to spot coring. The $\mathrm{Ca} \log$ for this interval (Fig. 1) exhibits a reasonable agreement with the core data. The base of the carbonates, at $320 \mathrm{mbsf}$, is obvious in both log and core data. The wide variability of discrete coulometer measurements makes this boundary appear more gradual in core than in log data. Visual core descriptions (Prell, Niitsuma, et al., 1989) also suggest a gradual transition, but other logs (e.g., velocity and neutron porosity) confirm that a sharp lithologic change occurs at 320 mbsf, with a continuing and more gradual transition below that depth. Within the carbonates, the logs and cores indicate that the intervals $70-140$ mbsf and $240-315$ mbsf have the highest carbonate content. In the intervening interval 140-240 mbsf, visual core descriptions indicate numerous beds of diatomaceous mud and a general dilution of the nannofossil ooze carbonate by diatoms. As a result, both the log and coulometer calcium records exhibit very high variability and generally lower calcium than in adjacent depth intervals.

Both the $\log \mathrm{Ca}$ and core carbonate data indicate that the lowest average $\mathrm{CaCO}_{3}$ is in the turbidites, below 320 mbsf. However, the $\mathrm{Ca} \log$ shows values for $350-405$ mbsf thatthough quite variable-are almost as high as 140-240 mbsf; in contrast, the core carbonate data are only rarely as low in the interval 140-240 mbsf as in the turbidites. The raw, unreprocessed Ca log (Prell, Niitsuma, et al., 19S9) is more consistent with core data in this respect; it is consistently lower in the turbidites than in the diatom-rich carbonates of 140-240 mbsf. This baseline difference between raw and reprocessed Ca logs is probably attributable to our arbitrary choice of a chlorine baseline for the chlorine repartitioning. The upper turbidites had perhaps the greatest variation in hole diameter of any sediments logged on Leg 117. Consequently, this interval is subject to major uncertainty concerning the relative proportions of the $\mathrm{Cl}$ smallscale variability that are due to hole size and partitioning problems. Normally, a caliper log would help to resolve this question, but no reliable caliper was obtained at Site 731. Indeed, none has been available to ODP until Leg 125 .
Of the two Site 731 intervals with high-resolution XRF sampling, the upper interval (interval 6 of Table 4) is in nannofossil ooze and the lower interval (interval 7) is in the low-carbonate terrigenous turbidites. The ratio of mean $\mathrm{Ca}$ concentration for the lower interval to that of the upper interval is $\mathbf{0 . 2 2}$. For comparison, the $\mathrm{Ca} \log$ yields a ratio of 0.43 . Clearly, the $\mathrm{Ca} \log$ readily detects the major difference in $\mathrm{Ca}$ concentration between these two intervals. The difference in ratios is probably attributable to the overestimation of the relative amount of $\mathrm{Ca}$ in the turbidites.

The results from the Conoco test well indicated a Ca precision of 2\% (Chapman et al., 19S7). As previously discussed, ODP geochemical logging precision might be expected to be less than at the Conoco test well. Six of our seven intervals have a standard deviation of XRF Ca concentrations of more than $2 \%$ (Table 5). Thus we might anticipate that the general character of XRF Ca variations would be detectable by the $\mathrm{Ca}$ log. Figure 3 compares the two data types for the seven depth intervals. Qualitatively, the character match between core and log $\mathrm{Ca}$ variations ranges from fair to very good (Table 5).

The correlation coefficient $(R)$ between the two data types is a more quantitative comparison. However, $\mathrm{R}$ is severely degraded by any residual depth shifts. Longer depth intervals are more likely to contain differential depth shifts within the XRF data due to incomplete core recovery. As a result, the longer depth intervals generally have lower $\mathrm{R}$ (Table 5), particularly for our best determined log: $\mathrm{Ca}$. In particular, the longest interval (interval 5) shows a very good match of XRF and log Ca below $257 \mathrm{mbsf}$ but a poor match above this depth (Fig. 3). The resulting correlation coefficient is only 0.17 . A residual depth shift of about $1 \mathrm{~m}$ for the XRF data above $257 \mathrm{mbsf}$ would substantially improve the match. We note that $257 \mathrm{mbsf}$ occurs within a core with nominal $101 \%$ recovery and with some core disturbance, but we do not feel that the character match between XRF and logs above 257 mbsf is sufficient to confidently infer that a shift is present.

Based on the correlation coefficient between XRF and log $\mathrm{Ca}$, intervals 1-4 strongly confirm the ability of the Ca $\log$ to reliably detect $\mathrm{Ca}$ variations of $2 \%-7 \%$. The correlation coefficient calculations of Table 5 exclude the first three XRF measurements of interval 3; the previously discussed log normalization problem at these depths yields absurdly high log $\mathrm{Ca}$ values there (Fig. 3). Intervals 4 and 7 exhibit a good character match, but gradual drift in the $\mathrm{Ca} \log$ baseline is evident as a gradual downhole increase in $\mathrm{Ca}$ values that is not confirmed by the $\mathrm{XRF}$ data. A single very low-Ca XRF point in interval 6 is not detected by the log. This anomalous point is evident on plots of other elements as well (Figs. 4-9) without a corresponding log response, and we suspect that it is a very thin bed below the resolving power of the logs.

Based on these comparisons of $\mathrm{Ca}$ logs with both coulometer measurements of $\mathrm{CaCO}_{3}$ and XRF measurements of $\mathrm{Ca}$, we conclude that the $\mathrm{Ca} \log$ does detect $\mathrm{Ca}$ variations larger than about $2 \%$. However, some residual repartioning problem can cause drift of the $\mathrm{Ca} \log$. We suspect that partitioning between $\mathrm{Cl}$ and $\mathrm{Ca}$ yields is the problem, and we think that further processing tests on these or other geochemical logs are warranted.

\section{Silicon}

The change from nannofossil ooze in interval 6 to terrigenous turbidites in interval 7 causes an increase in average silicon abundance from $7 \%$ to $23 \%$ (Table 5). This increase is much larger than the estimated $\mathrm{Si} \log$ precision of $1.5 \%$ (Chapman et al., 1987) and should be quite evident on the Si log. The Si log exhibits a factor of 2.1 increase, quite significant but less than the factor of 3.2 increase in XRF Si abundance. 
Table 5. XRF elemental results by interval. Both subjective (fit) and objective (correlation coefficient $\mathbf{R}$ ) matches to logs are shown.

\begin{tabular}{|c|c|c|c|c|c|c|c|c|}
\hline \multicolumn{2}{|c|}{$\begin{array}{l}\text { Top depth } \\
\text { End depth } \\
\text { Number }\end{array}$} & \multirow{2}{*}{$\begin{array}{c}92.74 \\
102.85 \\
30 \\
20.5\end{array}$} & \multirow{2}{*}{$\begin{array}{c}208.65 \\
233.68 \\
93\end{array}$} & \multirow{2}{*}{$\begin{array}{c}381.25 \\
386.10 \\
17\end{array}$} & \multirow{2}{*}{$\begin{array}{c}178.55 \\
182.80 \\
18 \\
21.4\end{array}$} & \multirow{2}{*}{$\begin{array}{l}247.75 \\
277.35 \\
122 \\
21.5\end{array}$} & \multirow{2}{*}{$\begin{array}{c}299.53 \\
308.50 \\
30 \\
29.3\end{array}$} & \multirow{2}{*}{$\begin{array}{c}341.60 \\
360.15 \\
59 \\
6.6\end{array}$} \\
\hline $\mathrm{Ca}$ & Mean & & & & & & & \\
\hline & $\sigma$ & 1.6 & 2.6 & 7.3 & 3.3 & 3.9 & 6.2 & 5.2 \\
\hline & fit & G & VG & F & VG & G & F & G \\
\hline & $\mathbf{R}$ & 0.47 & 0.61 & 0.72 & 0.56 & 0.17 & 0.23 & 0.26 \\
\hline \multirow[t]{4}{*}{$\mathrm{Si}$} & Mean & 11.6 & 9.9 & 12.2 & 10.9 & 11.6 & 7.0 & 22.7 \\
\hline & $\sigma$ & 1.2 & 2.5 & 6.0 & 2.2 & 2.5 & 4.1 & 3.9 \\
\hline & fit & $\mathrm{F}$ & G & $\mathbf{P}$ & G & F & $\mathbf{F}$ & F \\
\hline & $\mathbf{R}$ & 0.54 & 0.49 & 0.33 & 0.27 & 0.04 & 0.14 & 0.32 \\
\hline \multirow[t]{4}{*}{$\mathrm{K}$} & Mean & 0.89 & 0.74 & 0.71 & 0.76 & 0.79 & 0.78 & 2.55 \\
\hline & $\sigma$ & 0.21 & 0.35 & 0.84 & 0.51 & 0.58 & 0.90 & 1.24 \\
\hline & fit & $\mathrm{F}$ & $\mathrm{F}$ & G & G & F & F & $\mathbf{P}$ \\
\hline & $\mathbf{R}$ & 0.26 & 0.14 & 0.08 & 0.37 & 0.14 & -0.21 & -0.05 \\
\hline \multirow[t]{4}{*}{$\mathrm{Ti}$} & Mean & 0.27 & 0.21 & 0.21 & 0.21 & 0.22 & 0.13 & 0.47 \\
\hline & $\sigma$ & 0.03 & 0.04 & 0.09 & 0.05 & 0.06 & 0.08 & 0.09 \\
\hline & fit & P & F & F & $\mathrm{G}$ & $\mathrm{F}$ & $\mathbf{P}$ & P \\
\hline & $\mathbf{R}$ & -0.01 & 0.09 & 0.64 & 0.46 & 0.11 & 0.06 & -0.05 \\
\hline \multirow[t]{4}{*}{$\mathrm{Fe}$} & Mean & 1.91 & 1.77 & 1.82 & 1.91 & 1.93 & 1.42 & 5.74 \\
\hline & $\sigma$ & 0.08 & 0.16 & 0.29 & 0.18 & 0.22 & 0.44 & 0.50 \\
\hline & fit & $\mathrm{F}$ & $\mathbf{P}$ & $\mathbf{P}$ & $\mathrm{F}$ & $\mathrm{F}$ & G & $\mathbf{P}$ \\
\hline & $\mathrm{R}$ & 0.04 & -0.19 & 0.23 & 0.48 & -0.04 & 0.28 & -0.37 \\
\hline \multirow[t]{4}{*}{$\mathrm{S}$} & Mean & 0.59 & 0.84 & 1.22 & 0.61 & 0.47 & 0.018 & 0.020 \\
\hline & $\sigma$ & 0.10 & 0.16 & 0.29 & 0.09 & 0.11 & 0.006 & 0.011 \\
\hline & fit & P & P & G & G & F & $?$ & $?$ \\
\hline & $\mathrm{R}$ & 0.48 & 0.02 & 0.69 & 0.33 & 0.12 & 0.28 & 0.27 \\
\hline \multirow[t]{4}{*}{$\mathrm{Al}$} & Mean & 2.74 & 2.27 & 2.21 & & & & \\
\hline & $\sigma$ & 0.23 & 0.48 & 0.87 & & & & \\
\hline & fit & $\mathrm{F}$ & G & F & & & & \\
\hline & $\mathbf{R}$ & 0.18 & 0.56 & 0.55 & & & & \\
\hline
\end{tabular}

Based on XRF, six of the seven intervals of Table 5 have standard deviations of $\mathrm{Si}$ abundance greater than $1.5 \%$. Thus one anticipates that the $\mathrm{Si}$ log should be able to capture much of the small-scale variability detected by XRF in these intervals. The qualitative match between XRF and $\log \mathrm{Si}$ character in these intervals (Fig. 4) ranges from fair to good. Correlation coefficients are moderately good, with the exceptions of intervals 5 and 6 . Interval 5 does not exhibit a very convincing match between XRF and $\log \mathrm{Si}$, even below the previously discussed zone with a possible depth-shift problem. Intervals 2,6 , and 7 show several $\mathrm{Si} \log$ drops to near zero that are inconsistent with the XRF results; these excursions are probably indicative of a partitioning problem in the inversion of GST spectra. In summary, Figure 4 shows that core silicon variations of $2 \%-6 \%$ are often but not consistently detected by the $\mathrm{Si} \log$.

\section{Potassium}

The change in potassium content at the base of the carbonates at Site 731 is clearly detectable in the NGT potassium log (Fig. 1). The mean XRF K content for the lower XRF interval at this site is 3.3 times that for the upper interval. The corresponding $\mathrm{K} \mathrm{log}$ intervals give a ratio of $\mathbf{2 . 6}$, demonstrating that this major change in $\mathrm{K}$ is recorded by the $\log$ with reasonable fidelity.

Six of the seven intervals of Figure 5 have a higher standard deviation of XRF-based $\mathrm{K}$ than the nominal $0.25 \%$ precision cited by Chapman et al. (1987). Thus one might anticipate a relatively good match of XRF and log small-scale character for K. However, the character match ranges from poor to good and is mostly only fair (Fig. 5). Correlation coefficients (Table 5) are generally low and positive, with the poorest fit occurring in interval 7, which has an XRF standard deviation 5 times the nominal resolution limit. We suspect that the poor $\mathrm{K} \log$ for interval 7 results from borehole effect. This interval has wide swings in borehole diameter due to washouts, but we are unable to apply the normal hole-size corrections to the NGT logs because no reliable caliper was obtained on Leg 117.
Intervals 1-3, from Site 723, exhibit only a fair visual match of $\mathrm{K} \log$ to XRF K; correlation coefficients are also low. Further, replicate NGT logging passes had only a fair agreement. In contrast, replicate uranium logs obtained simultaneously by the NGT showed very good agreement and the highest $U$ concentrations that we have seen (Prell, Niitsuma, et al., 1989). Apparently the anomalously high $\mathrm{U}$, associated with extremely high concentrations of organic matter at this site, exaggerates slight problems in the inversion of the natural gamma spectrum for $\mathbf{K}$, $\mathrm{Th}$, and $\mathrm{U}$. This possible inversion problem, coupled with a $\mathrm{K}$ variation only slightly higher than the nominal resolution of the NGT, results in the low-quality match with XRF.

Interval 6 has a visually good match of $\log \mathrm{K}$ with XRF with an indication of a slight, half-meter depth shift between cores and logs; this depth shift is sufficient to degrade the correlation coeffient to a negative value. The $\mathrm{K} \log$ from interval 7 is the only element log from this interval that shows some evidence of partly detecting the very thin bed represented by one XRF sample (Fig. 5). However, the K logs of Figure 5 generally have lower vertical resolution than the GST element logs of Figures 3, 4, 6, and 7 , because of the variable-length Kalman filter applied by Schlumberger processing. For example, the 2-m cyclicity of interval 5 appears to be only marginally resolved by the $\mathrm{K} \log$.

The surprisingly low quality of our $\mathrm{K}$ log match with XRF indicates a strong need for further evaluation of NGT K logs, on wells with either a relatively uniform hole size (e.g., basalt) or a reliable caliper log of hole size.

\section{Titanium}

The transition from carbonates to turbidites at Site 731 causes an increase in XRF titanium by a factor of 3.6. The Ti $\log$ gives a much lower ratio of 2.0 , but this difference is not surprising because the XRF-based difference is only $0.3 \%$, compared to a nominal $0.1 \%$ resolution of $\mathrm{Ti}$ logs (Chapman et al., 1987).

All seven intervals of Figure 6 are lower to much lower in XRF Ti than the nominal $0.1 \%$ resolution, so little to no match 

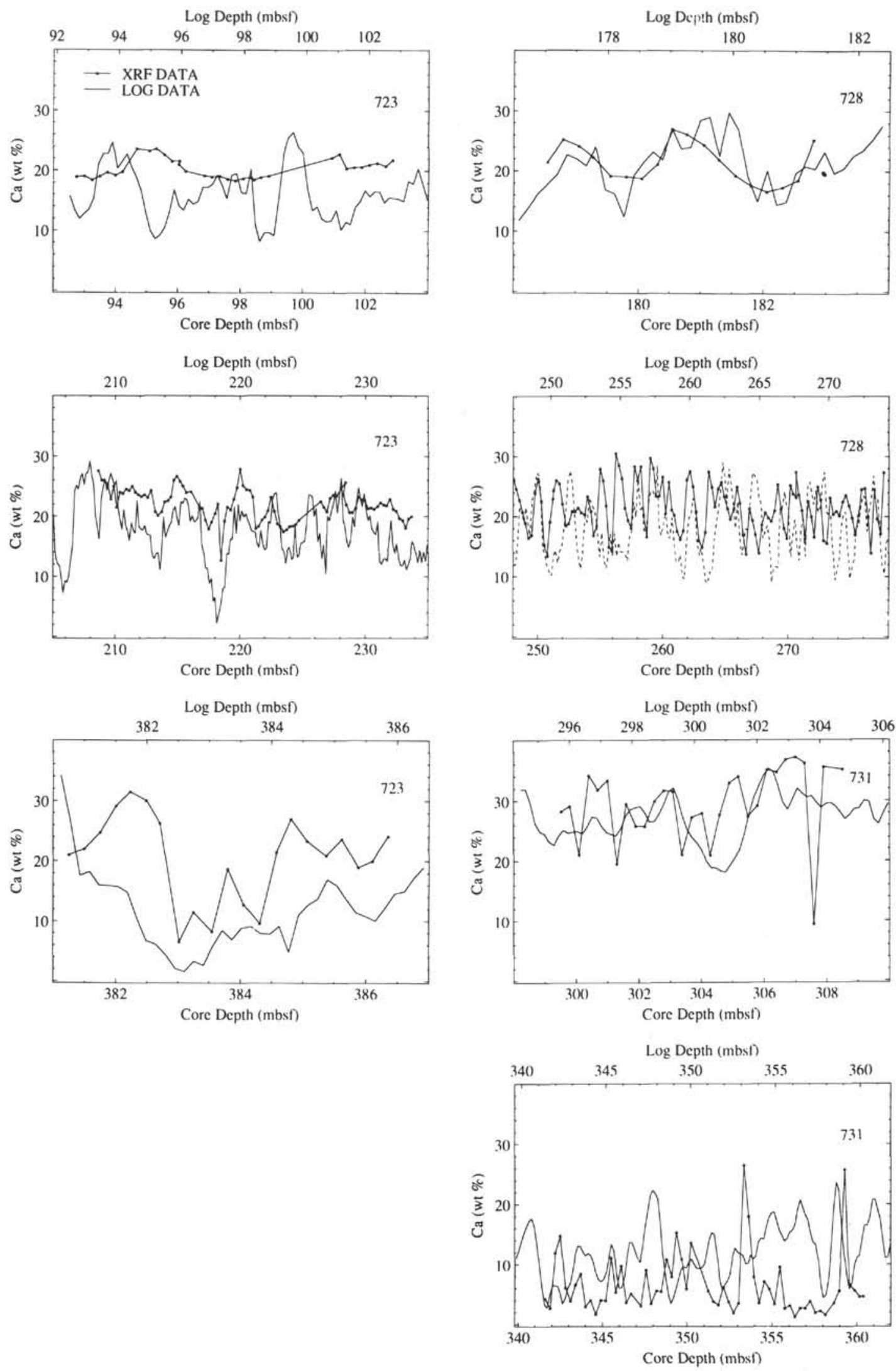

Figure 3. Comparison of XRF and geochemical log determinations of calcium, for the seven intervals of Table 1. Log data are indicated by solid or dashed lines with no symbols. Solid squares connected by solid lines indicate XRF data. The focus here is on the extent to which the character of the two data types agrees, not on absolute percentages. 

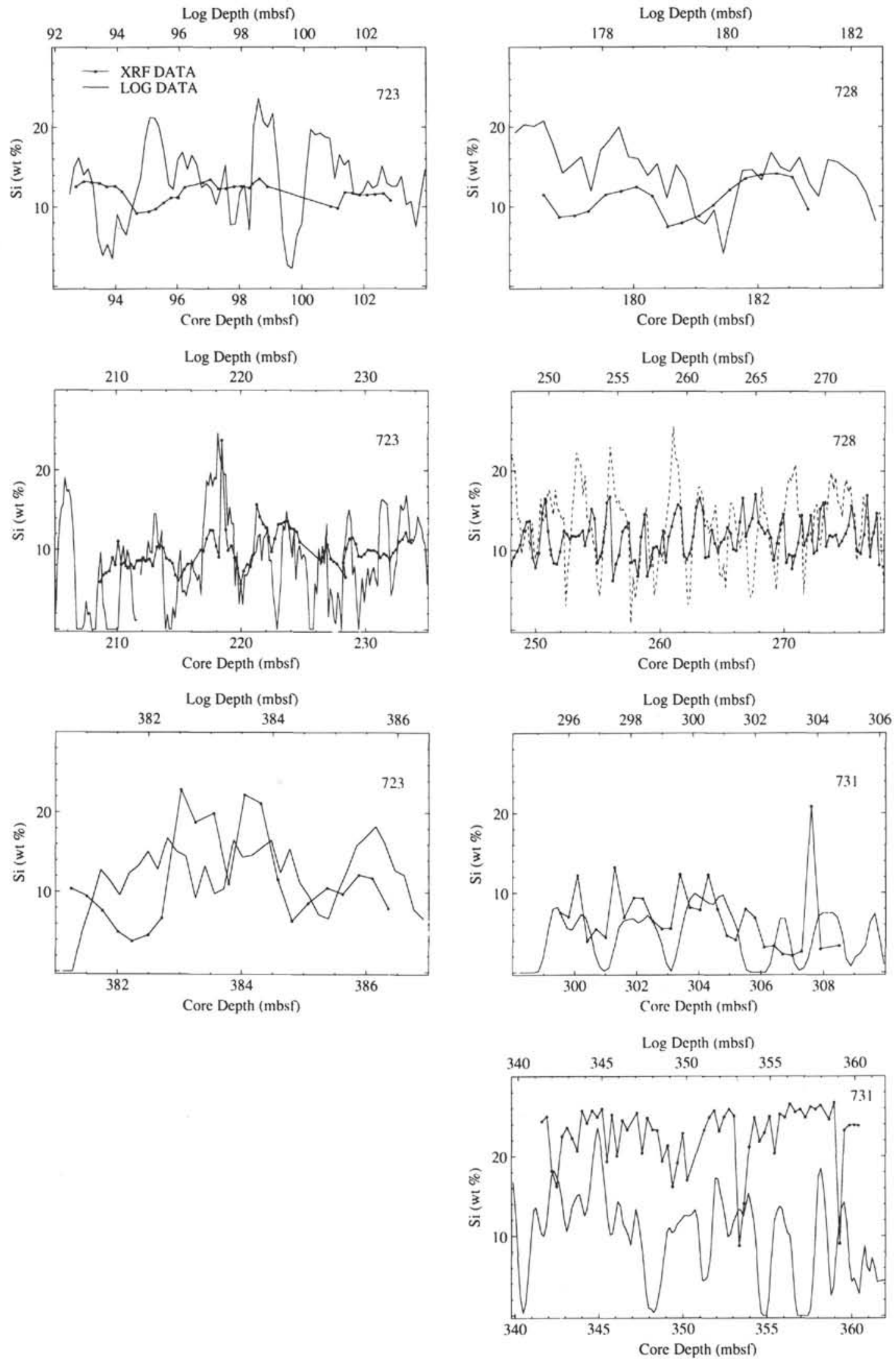

Figure 4. Comparison of XRF and geochemical log determinations of silicon, for the seven intervals of Table 1. See Figure 3 for explanation. 

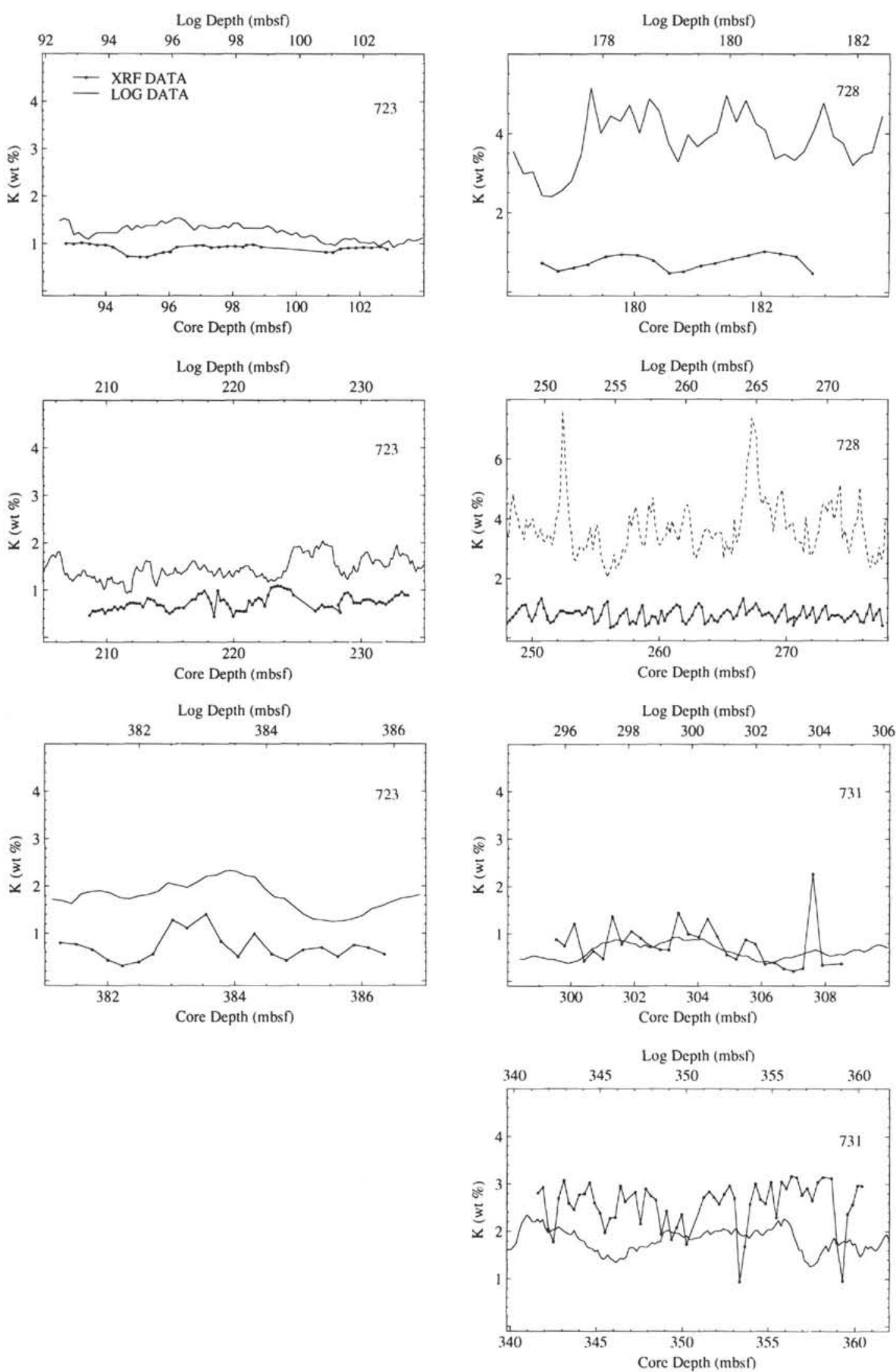

Figure 5. Comparison of XRF and geochemical log determinations of potassium, for the seven intervals of Table 1. See Figure 3 for explanation. 

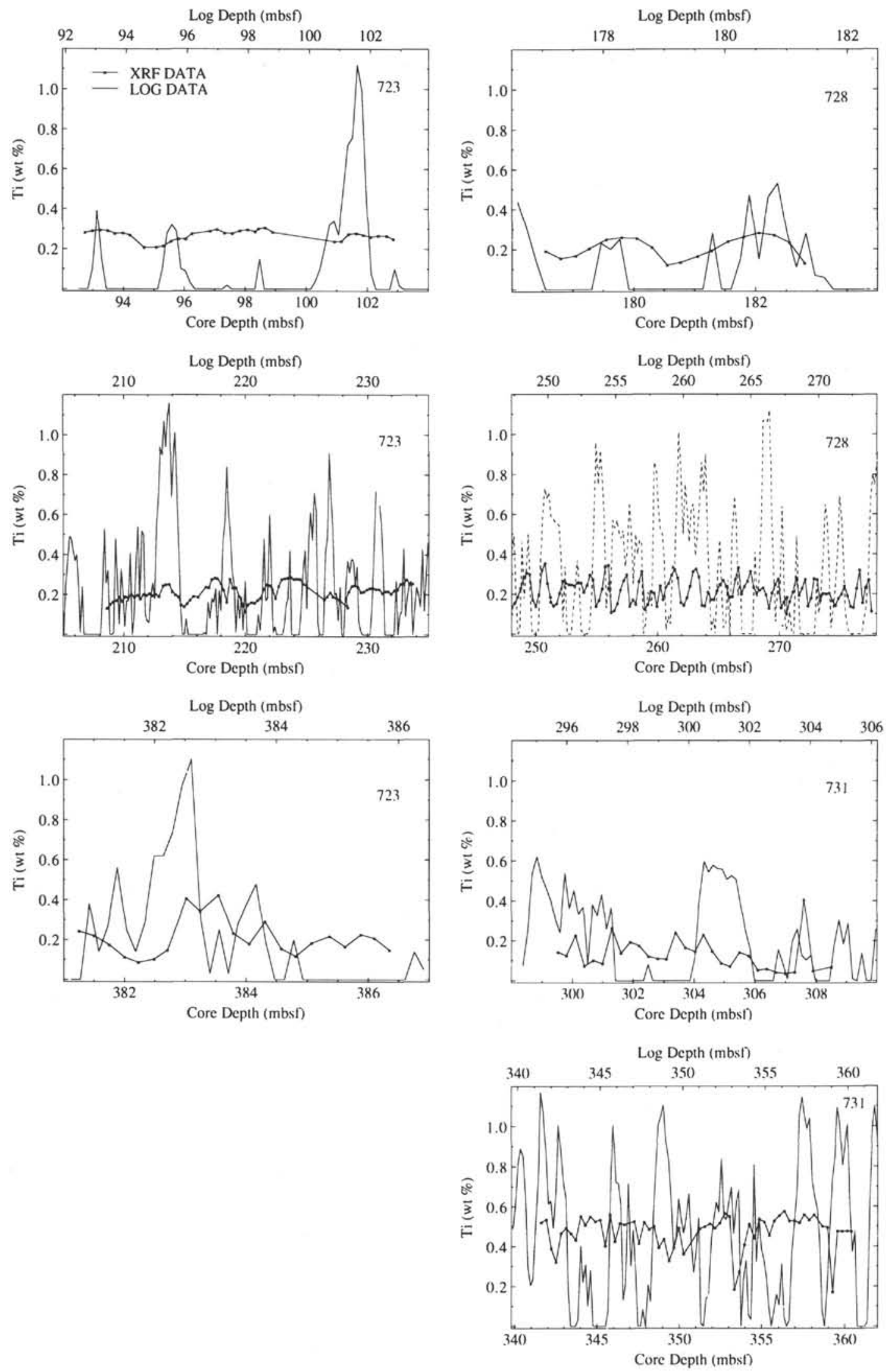

Figure 6. Comparison of XRF and geochemical log determinations of titanium, for the seven intervals of Table 1. See Figure 3 for explanation. 
of $\log$ to XRF is anticipated. The actual match is mostly poorfair, with near-zero correlation coefficients. Exceptions are intervals 3 and 4 , with good visual matches and high correlation coefficients of $0.5-0.6$, though the XRF-based variation in $\mathrm{Ti}$ is no higher in these intervals than in interval 7, where the match is only poor.

Titanium counts after $\mathrm{Cl}$ and $\mathrm{K}$ repartitioning often go negative; these negative counts are truncated at zero in Figure 6 . The repartitioning of all $\mathrm{K}$ that we employed lowered $\mathrm{Ti}$ counts too much, by effectively assuming that true $\mathrm{K}$ concentration is zero. The normal Schlumberger processing, which uses the NGT K log to estimate the amount of GST K to be repartitioned, probably would have resulted in a more reliable Ti log. However, the unusually low accuracy of our NGT K log might have limited this improvement, even if we had the complete geochemical suite of logs required for this type of processing. We conclude that geochemical logging has promise for detecting $\mathrm{Ti}$ variations of $<0.1 \%$, if partitioning interference with $\mathrm{K}$ (and possibly S) can be reduced with a reliable NGT K log. Stacking of replicate NGT runs might accomplish part of this needed NGT improvement; a reliable caliper for borehole correction probably would help even more.

\section{Iron}

The transition from carbonates to turbidites at Site 731 causes an increase in XRF iron by a factor of 4.0. The Fe log increases by a factor of only 1.6 over the same intervals. The major change is thus reliably detected in a qualitative sense, but an Fe calibration problem is suggested.

Within the two intervals of Site 731 (intervals 6 and 7), the variation observed in XRF measurements of $\mathrm{Fe}$ is about the same as the nominal Fe resolution of $0.5 \%$ (Chapman et al., 19S7) and the variations are expected to be marginally resolvable by the log. In contrast, intervals 1-5 (Sites 723 and 728) have much lower XRF Fe variability than $0.5 \%$ and are not expected to be resolvable. The qualitative match between XRF and $\log \mathrm{Fe}$ is poor to fair (Fig. 7), with correlation coefficients low and as often negative as positive (Table 5). Exceptions are interval 6 with a good visual fit and $R=0.28$ and interval 4 with a fair fit and $\mathrm{R}=0.48$. Thus the $0.5 \%$ resolution estimate of Chapman et al. (1987) appears to be reasonable for our ODP $\mathrm{Fe}$ logs.

\section{Sulfur}

The transition from carbonates to turbidites at Site 731 has no significant change in XRF sulfur content. Further, the XRF $\mathrm{S}$ at Site 731 is two orders of magnitude lower than the nominal $1.5 \% \log$ resolution for sulfur of Chapman et al. (1987). Thus, unlike the case for the other elements, this transition is not useful as a test of $S \log$ reliability.

Intervals 1-7 all have XRF S variations that are 1-2 orders of magnitude lower than the nominal $\mathrm{S} \log$ resolution. Thus it is unsurprising that intervals 1 and 2 have a poor character match of XRF and $\log \mathrm{S}$ (Fig. 8), as well as zero to negative correlation coefficients (Table 5). It is surprising that intervals $3-5$ have a fair to good character match, with fair to very good correlation coefficients (e.g., 0.69 for interval 3). This agreement may be an indication of poor partitioning rather than of unexpectedly very high resolving power of the $S \log$. Intervals 1 and 2 , with a poor match, have no XRF correlation between $\mathrm{S}$ and the noncarbonate elements, whereas intervals 3 and 4 with a good match do have a good XRF correlation between $\mathrm{S}$ and the noncarbonate elements. The sulfur concentrations from logs are much higher than the XRF sulfur concentrations, further suggesting a partitioning problem. Thus the "S log" may actually represent a poorly partitioned composite of at least one other non-carbonate element. However, a pilot repartitioning of all $\mathrm{S}$ to other ele- ments for Site 728 yielded revised $\mathrm{Si}, \mathrm{Fe}, \mathrm{Ti}$, and $\mathrm{Ca}$ logs that were almost identical to those of Figures 3, 4, 5, and 6; only the revised $\mathrm{Ca}$ log had any noticeable (but trivial) change in character.

\section{Aluminum}

Aluminum logging was undertaken only at Site 723. The XRF Al variations in intervals 2 and 3 (Table 5) are slightly less than the nominal $1 \% \mathrm{Al}$ resolution of Chapman et al. (1987), while variations within interval 1 are much less than we can expect to resolve. The character match of XRF and log $\mathrm{Al}$ ranges from fair to very good among the three intervals; high correlation coefficients of 0.56 and 0.55 are found for intervals 2 and 3. These data suggest that $\mathrm{Al}$ character is useful for variations of $>0.2 \%-0.4 \%$. However, our absolute accuracy is poor: both the mean $\mathrm{Al}$ and range of $\mathrm{Al}$ values based on logs are much too high in all three intervals of Site 723. Our absolute accuracy for $\mathrm{Al}$ is degraded by lack of a caliper and therefore of hole-size correction to the aluminum log.

\section{Geochemical Log Accuracy}

The element-by-element analyses above focus on the fidelity with which geochemical logs detect the character of geochemical variations within the formations. They do not consider accuracy, the extent to which the magnitudes of XRF abundances are duplicated by the geochemical logs. In a preceding section on geochemical log processing, we noted that the Leg 117 geochemical logs cannot be converted to weight percentages, because this conversion requires information on all major elements, obtainable only with both a $\log$ of photoelectric effect and the full suite of geochemical logs. Because many sites other than those on Leg 117 do not obtain all logs, a brief discussion is warranted of the accuracy resulting from the less reliable alternative processing utilized here. We focus on a comparison between logs and XRF of the average and within-interval variability for each element. Means and standard deviations could be used, but qualitative examination of Figures 3-9 is adequate.

Calcium concentrations (Figs. 3 and 10) appear to be estimated fairly well by our processing procedure. Both the average concentration and the intra-interval ranges of concentrations are generally comparable for logs and XRF. Exceptions are log variations that are too high in interval 1 , concentrations that are too low in interval 3, and the previously-discussed too-high $\mathrm{Ca}$ concentrations of interval 7.

Average silicon concentrations (Fig. 4) from logs also agree with XRF. Notable exceptions are the drops to zero in intervals 2 , 6, and 7, attributable to occasional partitioning problems. Also, silicon concentrations are too low in interval 7, because of the too-high $\mathrm{Ca}$ concentrations. The range of $\mathrm{Si}$ log variations within each interval is too high, particularly in interval 1 and whenever Si drops out to zero.

Potassium concentrations (Fig. 5) are too low in interval 6 and especially in interval 7 , where variable and enlarged hole sizes degrade the reliability of the $\mathrm{K} \log$ and introduce large, spurious variations in apparent $\mathrm{K}$ concentration. $\mathrm{K}$ concentrations are too high at Site 723 , possibly a result of the NGT inversion problem introduced by anomalously high uranium at this site.

Titanium log variations (Fig. 6) are much too high, and log abundances swing between values that are much too high and dropouts to zero concentration. An identical problem is evident for sulfur (Fig. 8), which is actually present in concentrations so low that it should yield constant and near-zero GST counts in all intervals.

Iron concentrations in logs (Fig. 7) are much too high, by a factor of 1.5-4. The inter-element range of $\mathrm{Fe}$ is reasonable in most intervals (an exception is interval 1). A baseline shift is implied, but its source is unknown. 

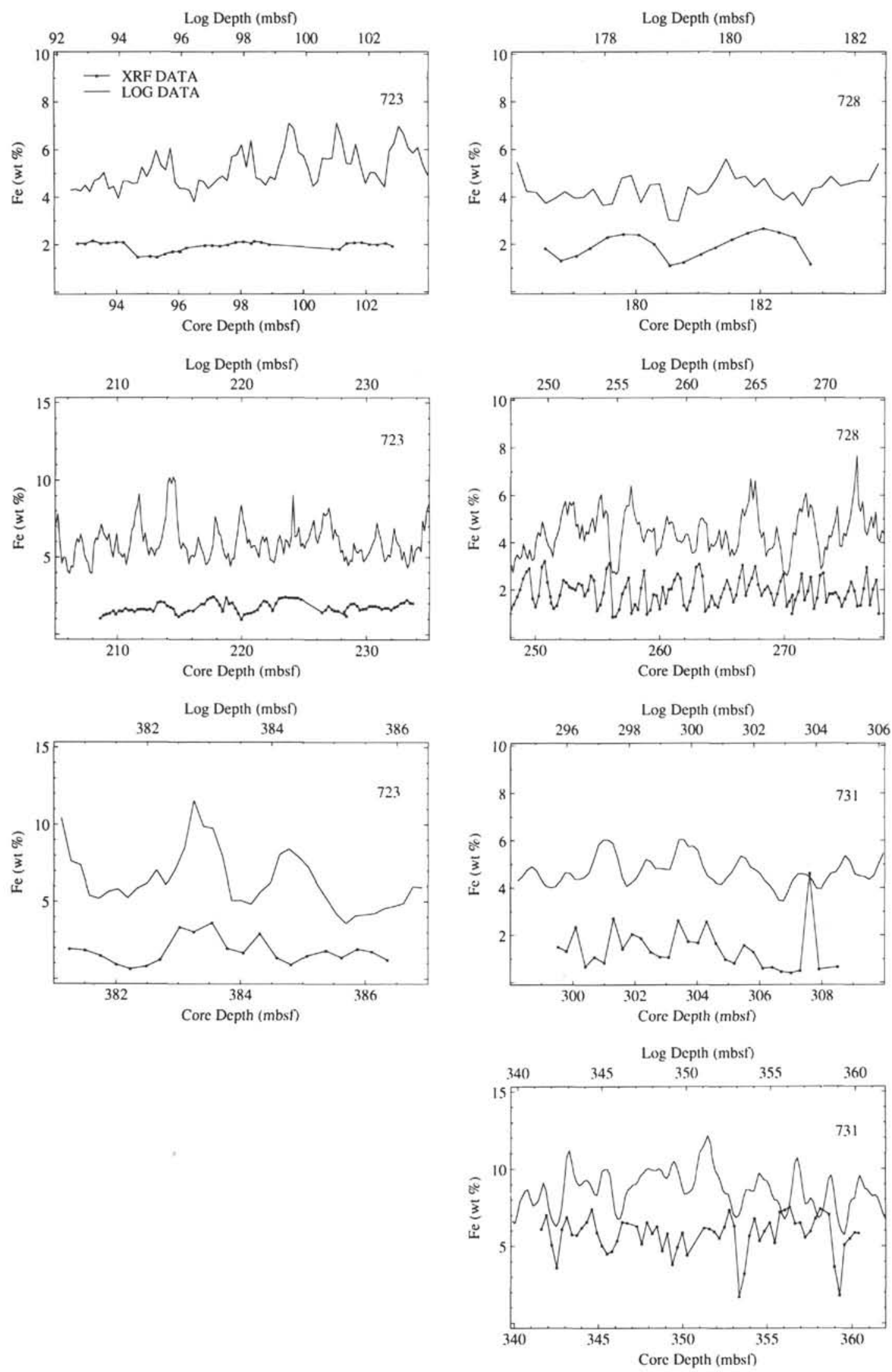

Figure 7. Comparison of XRF and geochemical log determinations of iron, for the seven intervals of Table 1. See Figure 3 for explanation. 

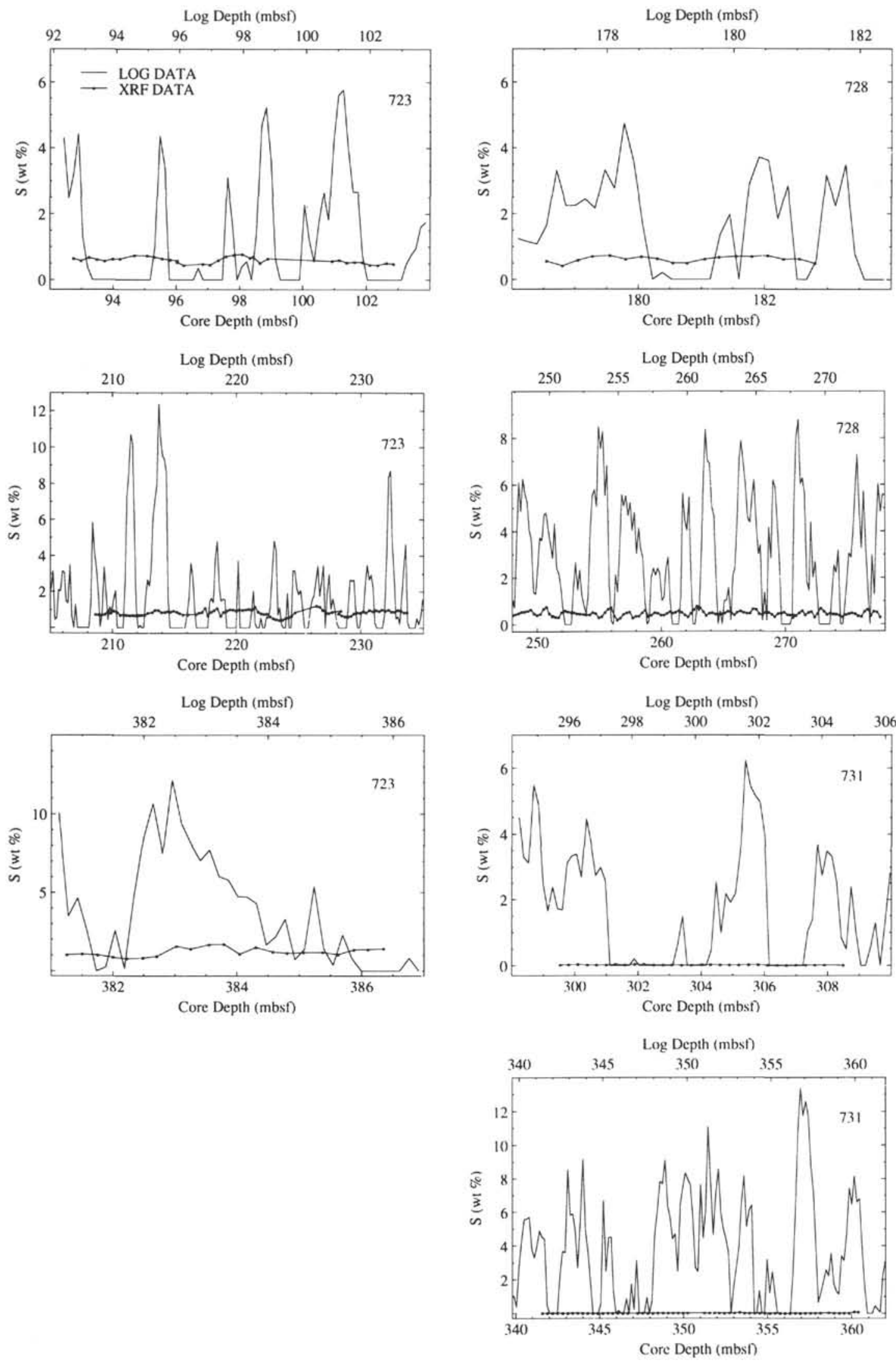

Figure 8. Comparison of XRF and geochemical log determinations of sulfur, for the seven intervals of Table 1. See Figure 3 for explanation. 

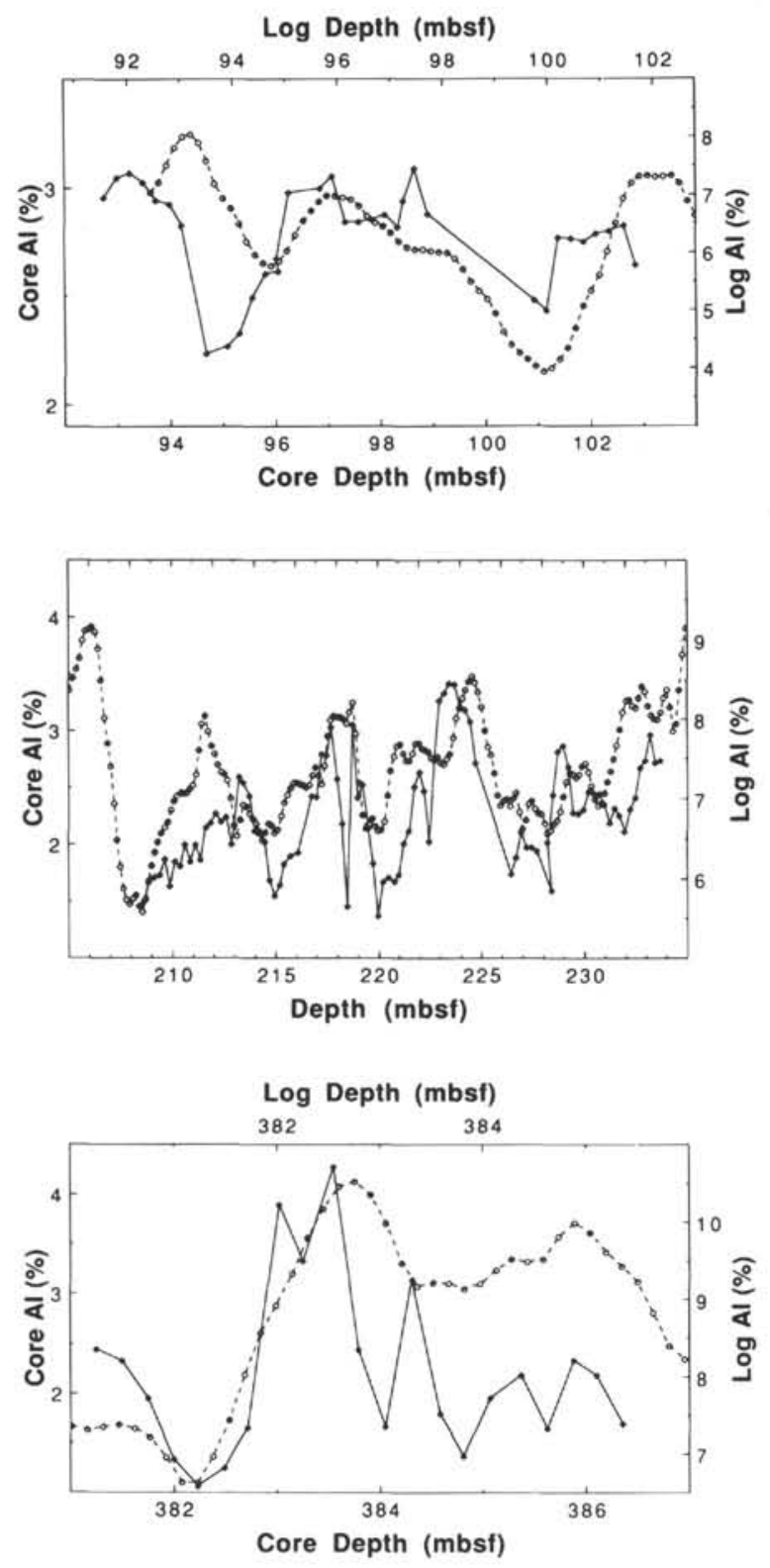

Figure 9. Comparison of XRF (solid lines) and geochemical log (dashed lines) determinations of aluminum, for the first three intervals of Table 1.

Aluminum concentrations (Fig. 9) are independent of the processing procedures used to refine GST elements. The aluminum clay tool is a tool very recently developed by Schlumberger, obtained by ODP partly because ODP provides a fruitful environment for testing its reliability. It is claimed to directly determine volume percent aluminum. However, at Site 723 the range of $\mathrm{Al}$ log values is much too high, and average $\mathrm{Al}$ log values are too high by a factor of $2-4$. In view of the tool's demonstrated ability to reliably capture the character of small variations in formation aluminum, further calibration of the tool is certainly warranted. We cannot conclude on the basis of only one site that the log always overestimates formation aluminum concentrations.

With a combination of geochemical logs and a few XRF samples from the same site, one can greatly improve the geochemical log accuracy by calibrating the logs. Such a calibration is clearly warranted for the three sites of this study. However, it
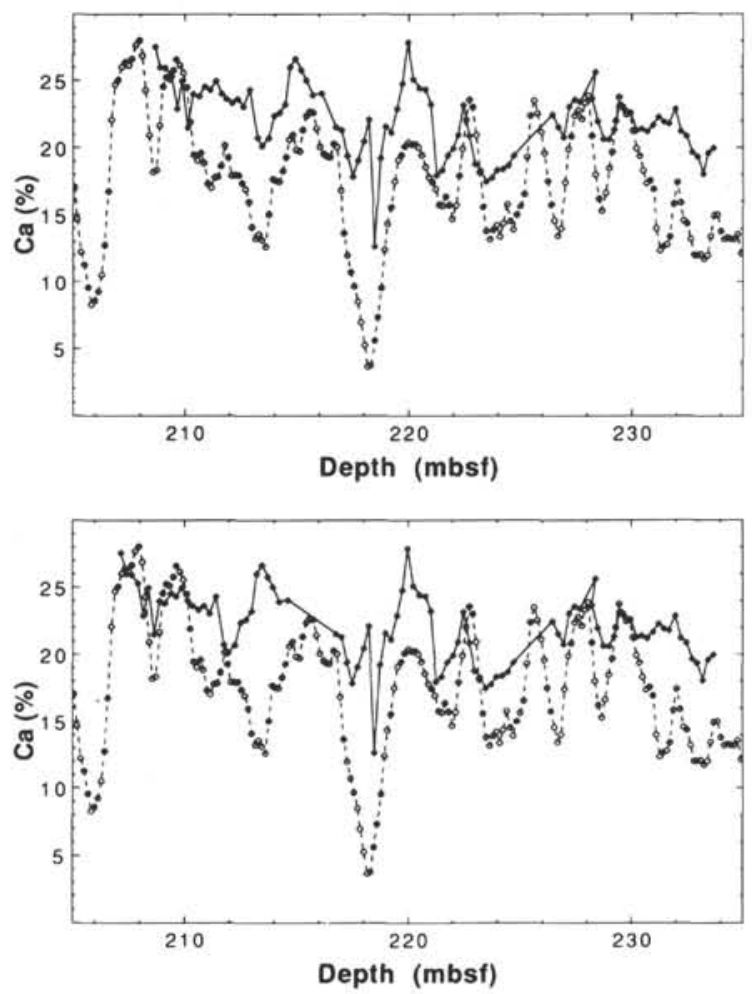

Figure 10. Comparison of XRF (solid lines) and geochemical log (dashed lines) determinations of calcium, for interval 2 of Table 1. Bottom: using the ODP convention of "hanging" core recovery from the top of the cored interval leads to a systematic mismatch of XRF and log character above 216.5 mbsf. Instead of assuming that incomplete core recovery is confined to the bottom of the cored interval, the top figure assumes that missing core was from the top of the cored interval.

is beyond the scope of the present study, and ideally it should be undertaken only after alternative processing schemes for GST data are compared, in order to reduce GST partitioning problems.

\section{INTER-ELEMENT VARIATIONS}

Examination of inter-element variations within both the XRF and $\log$ datasets offers a second method of evaluating geochemical log quality. This approach avoids a perennial problem of overlays of logs and core data as a function of depth: uncertain and variable depth shifts between cores and logs. It also reduces the problem of comparing character of log and XRF data, when the two datasets have different means and standard deviations (this difference does not affect the correlation coefficients of Table 5). A disadvantage of the method that we will use is that it does not consider the magnitude of elemental variances and whether they are much lower or higher than nominal log resolving power.

We applied principal components analysis to the XRF abundances of $\mathrm{Ca}, \mathrm{Si}, \mathrm{Fe}, \mathrm{Ti}, \mathrm{K}$, and (at Site 723) Al. We chose these elements because they are the same ones available from logs, with one exception. We excluded sulfur, because XRF analyses show that it often moves independently of the other elements and because the XRF concentrations are far below the nominal $\mathrm{S} \log$ resolution. We extracted principal components using the correlation matrix, effectively standardizing each element. For our purposes, this technique is much more appropriate than the alternative method which weights (or loads) components according to variance and which is therefore greatly dominated by calcium. XRF principal components were calculated for each of 
the seven intervals; as for the correlation coefficients previously described, we excluded the first four measurements from interval 1 and first three measurements from interval 3, so that XRF and log data were available for exactly the same intervals. Geochemical log principal components were independently determined for the seven intervals. The first two principal components for both the XRF analyses and log analyses are shown in Table 6.

For XRF data, the first principal component is very strong and consistent throughout the seven intervals, accounting for $84.6 \%-99.6 \%$ of the covariance of the 5-6 analyzed elements. Although identification of a principal component with a physical mechanism can be hazardous, there is no doubt that the first principal component here is caused by calcite dilution. Calcium is opposite in sign to all other elements, and we see loadings for all of the elements that are near the perfectly equal ideals of 0.408 for intervals $1-3$ (6 variables) and 0.447 for intervals 4-7 ( 5 variables). Because these principal components are effectively based on standardized variables rather than raw data, a given change in the dominant component $\mathrm{Ca}$ causes identical changes of opposite sign in all other components.

The second XRF principal component is more surprising and interesting. Although accounting for only $0.2 \%-13.2 \%$ of the covariance, it is very consistent among the seven intervals by principal component standards. This component is a silicon dilution effect, attributable to quartz or opaline silica. Silicon is the most highly loaded of the variables, and it is opposite in sign to the other variables (except occasionally to Ti). The contributions of $\mathrm{Ti}, \mathrm{Fe}$, and $\mathrm{Al}$ vary somewhat between intervals and are not particularly well defined, but $\mathrm{Ca}$ and $\mathrm{K}$ are relatively consistent. Note that this is not simply a variation in rela- tive proportions of the two dominant elements $\mathrm{Ca}$ and $\mathrm{Si}$. Instead the first two principal components indicate that most mineralogical abundance changes are in the relative proportions of calcite and combined "other" minerals (first principal component), but occasionally there is a quartz influx (second principal component). The same conclusion can be inferred from crossplots. Figures 11-13 show crossplots for the three sites (combining intervals within each site), illustrating both calcite dilution and occasional excess $\mathrm{Si}$. We note, however, that the second principal component at Site 731 may be more complex than a simple quartz component; there $\mathrm{Si}$ and $\mathrm{Ti}$ are positively correlated (Table 6), and the turbidites of interval 7 have a particularly complex mineralogy (Prell, Niitsuma, et al., 1989).

The percent of variance accounted for by the first principal component is highest $(99.6 \%)$ in interval 6 , where the highest calcite concentrations are found (Table 5). However, the first principal component is not affected by total calcite concentration, and its relative strength in interval 6 probably results from a weak second principal component in the carbonates of Site 731 , the most pelagic of the seven intervals.

The sum of the two principal components accounts for $97.4 \%$ $99.8 \%$ of the covariance of XRF elements included in this analysis. Thus principal components analysis makes very strong predictions about the inter-element relations expected among geochemical log elements.

Principal components analysis of the geochemical logs (Table 6) indicates substantially different patterns than the preceding analysis of XRF data, though the analyzed elements and depth intervals are identical. The first principal component is less than half the strength of the first XRF principal component. It is recognizably calcite dilution, consistently loaded on

Table 6. Principal components analysis.

\begin{tabular}{|c|c|c|c|c|c|c|c|}
\hline Top depth & 92.74 & 208.65 & 381.25 & 178.55 & 247.75 & 299.53 & 341.60 \\
\hline End depth & 102.85 & 233.68 & 386.10 & 182.80 & 277.35 & 308.50 & 360.15 \\
\hline Number & 30 & 93 & 17 & 18 & 122 & 30 & 59 \\
\hline \multicolumn{8}{|l|}{ X-ray diffraction } \\
\hline$\%$ & 93.1 & 84.6 & 93.6 & 97.4 & 96.4 & 99.6 & 93.2 \\
\hline $\mathrm{Ca}$ & 0.406 & 0.401 & 0.408 & 0.452 & 0.449 & 0.447 & 0.460 \\
\hline $\mathrm{Si}$ & -0.412 & -0.338 & -0.380 & -0.435 & -0.440 & -0.448 & -0.448 \\
\hline $\mathrm{K}$ & -0.415 & -0.420 & -0.410 & -0.450 & -0.450 & -0.446 & -0.442 \\
\hline $\mathrm{Ti}$ & -0.417 & -0.429 & -0.416 & -0.448 & -0.446 & -0.447 & -0.450 \\
\hline $\mathrm{Fe}$ & -0.386 & -0.432 & -0.420 & -0.451 & -0.452 & -0.447 & -0.436 \\
\hline Al & -0.413 & -0.422 & -0.413 & & & & \\
\hline$\%$ & 4.4 & 13.2 & 6.1 & 2.3 & 2.2 & 0.2 & 5.3 \\
\hline $\mathrm{Ca}$ & 0.280 & 0.461 & 0.408 & 0.110 & 0.284 & 0.459 & 0.199 \\
\hline $\mathrm{Si}$ & -0.427 & -0.718 & -0.714 & -0.838 & -0.746 & -0.341 & -0.473 \\
\hline K & 0.269 & 0.315 & 0.386 & 0.275 & 0.324 & 0.778 & 0.485 \\
\hline $\mathrm{Ti}$ & -0.099 & 0.255 & 0.241 & 0.356 & 0.444 & -0.172 & -0.381 \\
\hline $\mathrm{Fe}$ & 0.784 & 0.152 & 0.109 & 0.289 & 0.264 & 0.200 & 0.597 \\
\hline Al & -0.203 & 0.315 & 0.323 & & & & \\
\hline$\% 1$ st +2 nd & 97.4 & 97.9 & 99.7 & 99.7 & 98.6 & 99.8 & 98.5 \\
\hline \multicolumn{8}{|l|}{ Geochemical logs } \\
\hline$\%$ & 39.9 & 41.8 & 49.1 & 42.9 & 47.1 & 40.7 & 48.6 \\
\hline $\mathrm{Ca}$ & 0.452 & 0.571 & 0.568 & 0.650 & 0.568 & 0.690 & 0.470 \\
\hline $\mathrm{Si}$ & -0.370 & -0.586 & -0.395 & -0.600 & -0.436 & -0.469 & -0.438 \\
\hline K & 0.444 & -0.254 & -0.412 & 0.225 & 0.420 & 0.005 & 0.473 \\
\hline $\mathrm{Ti}$ & -0.493 & -0.057 & -0.386 & -0.390 & -0.397 & -0.543 & -0.344 \\
\hline $\mathrm{Fe}$ & -0.445 & 0.250 & -0.323 & 0.127 & 0.390 & -0.094 & 0.500 \\
\hline Al & 0.142 & -0.447 & -0.313 & & & & \\
\hline$\%$ & 30.6 & 24.2 & 24.5 & 33.8 & 24.2 & 34.7 & 23.8 \\
\hline $\mathrm{Ca}$ & 0.516 & 0.291 & 0.152 & 0.216 & 0.281 & 0.116 & 0.302 \\
\hline $\mathrm{Si}$ & -0.507 & -0.008 & 0.449 & 0.171 & 0.538 & 0.124 & -0.658 \\
\hline $\mathrm{K}$ & -0.416 & -0.065 & 0.312 & -0.580 & -0.268 & -0.694 & -0.221 \\
\hline $\mathrm{Ti}$ & 0.007 & -0.734 & 0.316 & -0.441 & -0.708 & 0.153 & 0.609 \\
\hline $\mathrm{Fe}$ & 0.403 & -0.580 & -0.538 & -0.630 & -0.240 & -0.683 & -0.236 \\
\hline Al & -0.376 & 0.189 & -0.536 & & & & \\
\hline
\end{tabular}



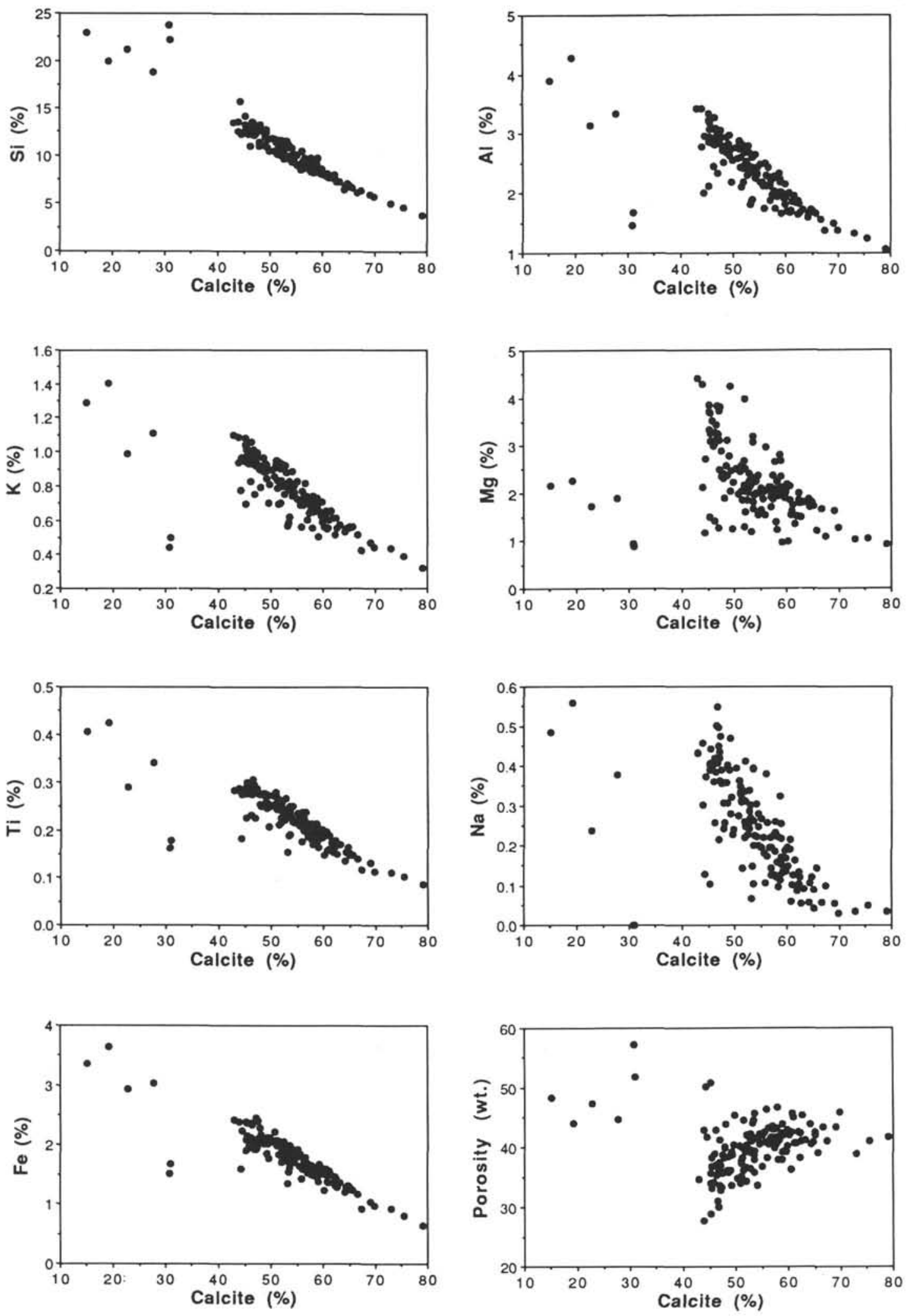

Figure 11. XRF element concentrations at Site 723, plotted as a function of XRF calcite percentage. Note the strong calcite dilution pattern for $\mathrm{Si}, \mathrm{K}, \mathrm{Ti}, \mathrm{Fe}$, and $\mathrm{Al}$, with occasional discrepant points indicative of quartz dilution. The poor correlation of porosity and calcite suggests that our procedure of repartitioning all chlorine character to other logged elements is unlikely to create an artificial correlation between calcium and other logged elements. 

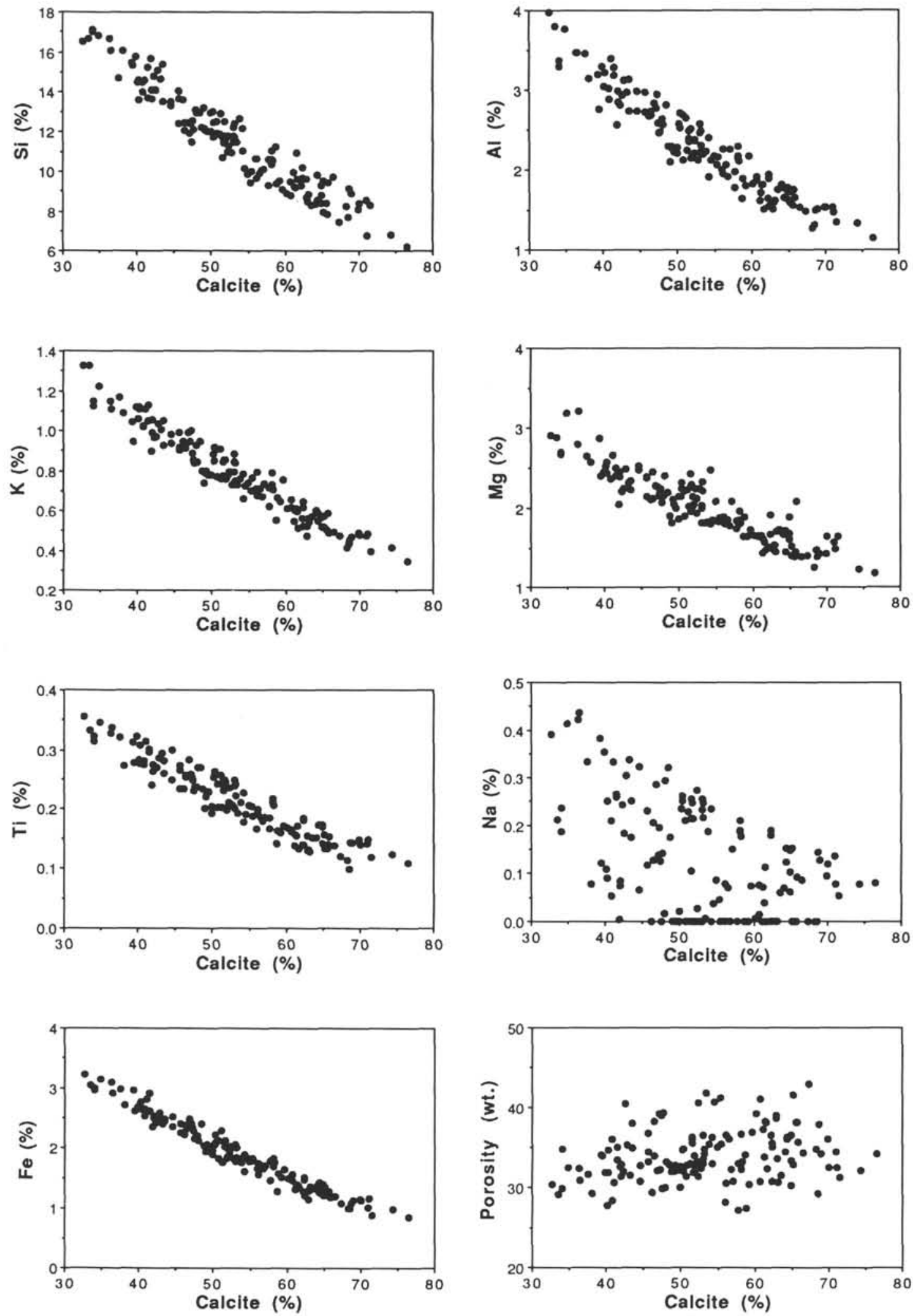

Figure 12. XRF element concentrations at Site 728, plotted as a function of XRF calcite percentage. Note the strong calcite dilution pattern for $\mathrm{Si}, \mathrm{K}, \mathrm{Ti}, \mathrm{Fe}, \mathrm{Al}$, and $\mathrm{Mg}$, with no obviously discrepant points indicative of quartz dilution. The poor correlation of porosity and calcite suggests that our procedure of repartitioning all chlorine character to other logged elements is unlikely to create an artificial correlation between calcium and other logged elements. 

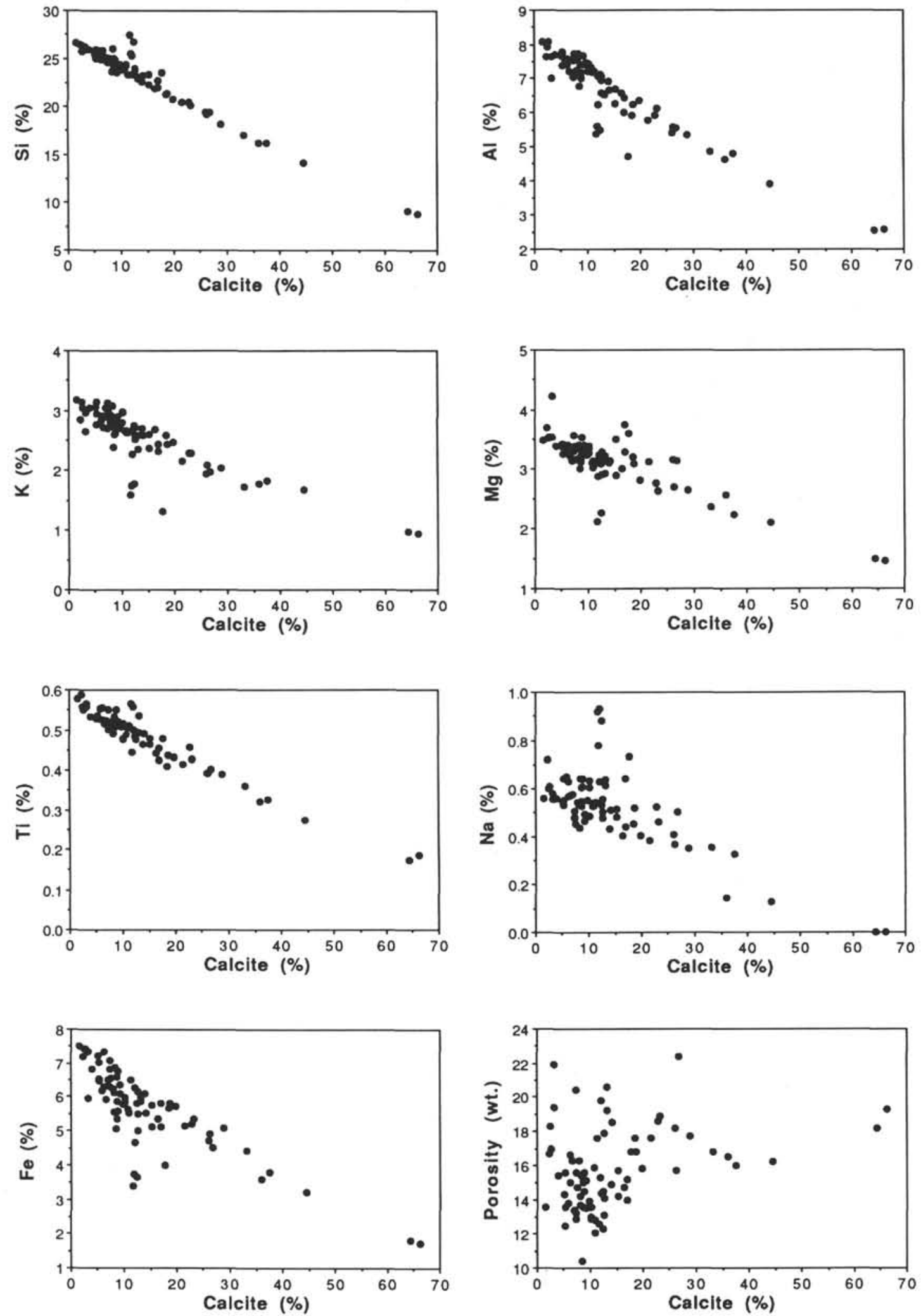

Figure 13. XRF element concentrations for the interval 340-450 mbsf at Site 731, plotted as a function of XRF calcite percentage. Note the strong calcite dilution pattern for $\mathrm{Si}, \mathrm{K}, \mathrm{Ti}, \mathrm{Fe}, \mathrm{Al}$, and $\mathrm{Mg}$, with occasional discrepant points indicative of quartz dilution. The poor correlation of porosity and calcite suggests that our procedure of repartitioning all chlorine character to other logged elements is unlikely to create an artificial correlation between calcium and other logged elements. 
$+\mathrm{Ca},-\mathrm{Si}$, and $-\mathrm{Ti}$. This pattern indicates that real silicon and titanium variations are in the $\mathrm{Si}$ and $\mathrm{Ti}$ logs, a conclusion previously reached on the basis of element plots vs. depth for $\mathrm{Si}$, but only tentatively concluded previously for Ti. Some inverse correlation between the dominant elements $\mathrm{Ca}$ and $\mathrm{Si}$ is introduced by the oxide normalization, but this effect cannot entirely account for the pattern seen here, because the inverse correlation is present, though weaker, before oxide normalization.

Only interval 3 shows the full calcite dilution pattern of positive $\mathrm{Ca}$ in the first principal component and negative loading for every other element. This interval also has the highest percentage of total covariance attributable to the first principal component $(49.1 \%)$. $\mathrm{K}$ and $\mathrm{Fe}$, which we previously inferred were poorly resolved by the logs in these intervals, give variable results in the first principal component and are often even positively correlated with $\mathrm{Ca}$. Al loading for the first principal component is inversely correlated with $\mathrm{Ca}$ for intervals 2 and 3 and near zero but positively correlated for interval 1 . This pattern is quite consistent with the Al vs. depth plots, in which we saw a good match to XRF in intervals 2 and 3 but only a fair match for interval 1 (which had $\mathrm{Al}$ variation far below the nominal resolving power of the logging tool).

If one calculates principal components from only the three logs $\mathrm{Ca}, \mathrm{Si}$, and $\mathrm{Ti}$ for each interval, the first principal component is again calcite dilution, accounting for $59 \%-70 \%$ of the covariance. Compared to the $88.5 \%-99.8 \%$ result from an XRF analysis of the same three elements, we may conclude that about two thirds $(59 \%-77 \%)$ of the observed log variations in $\mathrm{Ca}, \mathrm{Si}$, and $\mathrm{Ti}$ is real detection of lithologic variation and about one third is noise. Calcium consistently has the heaviest loading in this first log principal component and therefore the highest signal-to-noise ratio; titanium usually has the lowest and contributes most to the combined noise of the three elements.

The second principal component for the geochemical logs accounts for $24 \%-35 \%$ of the covariance (Table 6) and is not much weaker than the first principal component. Its physical origin is obscure. It appears to pick up the effect of $\mathrm{Ca}$ dilution on $\mathrm{K}$ and $\mathrm{Fe}$, since these elements are negatively loaded with respect to $\mathrm{Ca}$ in six of the seven intervals. However, only the portion of $\mathrm{Ca} \log$ variations that is real calcite dilution and is also uncorrelated with $\log \mathrm{Si}$ and $\mathrm{Ti}$ variation could be present in this second principal component. With loadings varying from interval to interval, it is not possible to assess the extent to which this component reflects calcite dilution, partitioning problems, and other systematic log errors.

\section{CONCLUSIONS}

The marly nannofossil chalks of Sites 723, 728, and 731 exhibit substantial geochemical variability at scales of 2-10 m. Xray fluorescence analysis, based on high-resolution sampling of seven intervals, demonstrates that calcite dilution is the primary source of this variability; occasional quartz influxes are also detected. The geochemical logs $\mathrm{Ca}, \mathrm{Si}$, and $\mathrm{Ti}$ detect the calcite dilution with sufficient precision to permit interpretation of highfrequency variations in calcite content for intervals with no XRF data but with openhole geochemical logs-nearly the entire hole below 56-92 mbsf at the three sites.

Our comparisons of 398 XRF analyses with geochemical logs indicates that the reliability of geochemical logs varies substantially, within short intervals and particularly between sites. In general, the geochemical logs are capable of detecting changes in formation geochemistry that are larger than the following thresholds: $2 \%$ for $\mathrm{Ca}, 2 \%-6 \%$ for $\mathrm{Si}, 0.5 \%-1 \%$ for $\mathrm{K}, 0.1 \%$ for $\mathrm{Ti}, 0.5 \%$ for $\mathrm{Fe}$, and $0.4 \%$ for $\mathrm{Al}$.

Accuracy of geochemical log percentages requires both the full suite of geochemical logs and a log of photoelectric effect. We obtained this full suite only at Site 723; even there, we were prevented from determining $\mathrm{Mg}$ and thereby improving accu- racy of the other geochemical logs by an unknown problem in the log of photoelectric effect. Without the full suite, we can make only first-order estimates of elemental percentages. These estimates yielded reasonable accuracy only for $\mathrm{Ca}$ and $\mathrm{Si}$. Thus the character of geochemical logs is often more reliable than their magnitudes.

Both the processing of these geochemical logs and the comparison of them to XRF data suggest several ways in which the reliability of ODP geochemical logs could be increased. First, as already mentioned, ideally both the full suite of geochemical logs and a photoelectric effect log should be obtained. Second, a reliable caliper log would improve the NGT K log and thereby also improve GST K repartitioning. Third, improved partitioning of the GST spectra is needed. A boron sleeve to reduce $\mathrm{Cl}$ counts may help, the improved NGT K log will help, and further reprocessing experiments on the Leg 117 geochemical logs could lead to improved partitioning coefficients. We note that ODP does now have a reliable caliper and a boron sleeve for the GST. Thus geochemical logs obtained after Leg 125 should be examined to evaluate whether or not the quality of geochemical logs is now higher than we found for Leg 117.

\section{ACKNOWLEDGMENTS}

This project was made possible by Lamar Hayes, who obtained excellent core recovery on ODP Leg 117; the shipboard scientific and technical parties; samples provided by the Ocean Drilling Program; the highly efficient XRF analytical facilities of Oregon State University; and the U.S. Science Advisory Committee, grant TAMRF P.O. 20167. The U.S. Science Program associated with the Ocean Drilling Program is sponsored by the National Science Foundation and the Joint Oceanographic Institutions, Inc. Any opinions, findings and conclusions expressed in this publication are those of the authors and do not necessarily reflect the views of the National Science Foundation, the Joint Oceanographic Institutions, Inc., the Texas A\&M University, or Columbia University.

\section{REFERENCES}

Anderson, R. N., Dove, R. E., and Pratson, E., in press. The calibration of geochemical well logs in basalt, granite, and metamorphic rocks; and their use as a lithostratigraphic tool. Geol. Soc. London.

Chapman, S., Colson, J. L., Flaum, C., Hertzog, R. C., Pirie, G., Scott, H., Everett, B., Herron, M. M., Schweitzer, J. S., La Vigne, J., Querein, J., and Wendlandt, R., 1987. The emergence of geochemical well logging. Tech. Rev., 35:27-35.

Herron, M. M., 1986. Mineralogy from geochemical well logging. Clays Clay Miner., 34:204-213.

Hertzog, R., 1979. Laboratory and field evaluation of an inelastic-neutron-scattering and capture gamma ray spectroscopy tool. Soc. Pet. Eng., Pap. 7430.

Herzog, R. L., Colson, B., Seeman, M., O’Brien, H., Scott, D., McKeon, P., Wraight, J., Schweitzer, J., and Herron, M., 1987. Geochemical logging with spectrometry tools. Soc. Pet. Eng., Pap. 16792.

Lock, G. A., and Hoyer, W. A., 1971. Natural gamma ray spectral logging. Log Analyst, 12:3-9.

Lyle, M., Heath, G. R., Pisias, N., and Muehlhausen, L., 1987. Sedimentary sources and diagenesis in the Pacific Ocean through the mapping of sediment chemical compositions. Eos, 68:470. (Abstract)

Prell, W. L., Niitsuma, N., et al., 1989. Proc. ODP, Init. Repts., 117: College Station, TX (Ocean Drilling Program).

Schlumberger, 1988. Trans., Inter. Spectroscopy Geochem. Symp., Ridgefield, CT (Schlumberger-Doll Res.).

Scott, H. D., and Smith, M. P., 1973. The aluminum activation log. Log Analyst, 14:3-12.

Serra, O., Baldwin, J., and Quiren, J., 1980. Theory, interpretation and practical applications of natural gamma-ray spectroscopy. Trans. SPWLA 21st Annu. Logging Symp., Q1-Q30.

Date of initial receipt: 26 September 1989

Date of acceptance: 25 July 1990

Ms 117B-174 